

\title{
COMPLIANT ROBOTIC STRUCTURES
}

F1rst Report to DARPA

June 1, 1984 - May 31, 1985

James F. Wilson

Departar:at of Civil and Environmental Engineering

Duke UnI versity

Durham, North Carolina 27706

August, 1985

\begin{abstract}
Sponsored by the Defense Advanced Research Projects Agency (DOD), ARPA Order No. 5092. The views, oplnions, and findings contained in this report are those of the authors and should not be construed as an official Department of Defense position, policy, or decision, unless so designated by other official documentation.
\end{abstract}


REPORT SECUAITY CLASSIFICATION

Unclassified

SECURITY CLASSIFKATION AUTMORITY

DECLASSIFICATION IOOWNGRADING SCMEDULE

PERFORMING ORGANIZATION REPORT NUMDER(S)

DUKE-CE-001-85-1

\section{HD $-A \%$ L $/ 897$}

3. DISTRIBUTION IAVALLABLITY OF REPORT

This document is available to the U.S. public through NTIS, Springfield virainie 22161

S MONITORING ORGANIZATION REPORT NJMBER(S)

\section{NAME OF DERFORMING ORGAN ZATION \\ Duke University \\ 7a. NAM! ?' MONITORING ORGANIZATION \\ ONRRR}

C. ADDRESS (City. Stute, and 210 Cede)

Durham, N. C. 27706

. NAME OF FUNDING/SPONSORING

onganization

DARPA (DOD)

76. ADDRESS (Civy, Stote, and ZIP Code) 2060 o'keefe Building

Atlanta Georoia 30332

Bb OFFICE SYMBOL 9. PROCUREMENT INSTRUMENT IDENTIFICATION NUMBER (if applicable)

MDA903-84-C-0243

Georgia Institute of Technology

ADDRESS (Ciny, Stare, and 2IP Code)

DARPA/DSO

1400 Wilson Blva.

Arlington, Virginia 22209

T. TITLE (Include Securrity Classification)

Compliant Robotic structures

2. PERSONAL AUTHOA(S)

Wilson, James $F$.

\begin{tabular}{l|l}
\hline 3a. TYPE OF REPORT & 13b. TIME COVEREO
\end{tabular}

flifirst year report FROM $6 / 1 / 84$ TO $5 / 31 / 8$

14. DATE OF REPORT (Year, MONTh, DOY)

15. PAGE COUNT WW. SUPPLEMENTARY NOTATION

10. SOURCE OF FUNDING NUMEERS

\begin{tabular}{l|l|l|l}
$\begin{array}{l}\text { PROGRAM } \\
\text { ELEMENT NO. }\end{array}$ & $\begin{array}{l}\text { PROJECT } \\
\text { NO. }\end{array}$ & $\begin{array}{l}\text { TASK } \\
\text { NO }\end{array}$ & $\begin{array}{l}\text { WORK UNIT } \\
\text { ACCESSION NO }\end{array}$
\end{tabular}

\section{COSATI CODES}

cosati codes

\begin{tabular}{|l|l|l|} 
FIELD & GROUP & SUB-GROUP \\
\hline
\end{tabular}

18 SUBJECT TERMS (Continue on reverse if necessary and identity by b/ock number) Bellows, cylindrical shells, manipulators, nonlinear materials, orthotropic shells, robotics

ABSTRACT (Continue on reverse if necessory and identify by block number?

tA compliant robotic structure is one or more continuously flexible arms Whthat can be controlled to manipulate objects. A typical arm is comprised of forthotropic tube elements placed end-to-end where each element is designed to Hmove in a particular way (bend, twist or extend) when pressurized.

The results of the first year studies are reported in three chapters. Chapter I focuses on the mechanics and structure of an elephant trunk. Chapter II involves the analysis of computer controlled compliant element Wsystems that bend and coil similar to elephant trunks. Chapter III focuses popon the analysis of compliant orthotropic tube elements that can be used as 00 torsion actuators when pressurized.

The 20 D!stribution IAVALLABILITY OF AESTRACT

$\because$ DunClassifie DNUNLIMITED $\square$ SAME AS apt

S. 220. NAME OF RESPONSIBLE INDIVIDUAL

hil Iiam E. Isler

DiIC USERS

21. ABSTRACT SECURITY
unclassified

22b. TELEPHONE (InClude Ared COOE) $22 \mathrm{C}$ OFfICE SYMEOL (202) $694-3624$ 


\section{SUMMARY}

In the present context, a compliant robotic structure is one or more continually flexible beams whose motion can be controlled to manipulate objects. Typically, a single beam is comprised of many hollow tube elements placed end-to-end, where each tube element moves in a particular way when pressurized with alr. Each tube element, made of a reinforced polymeric material, is designed with directional stiffness properties so that tube motion (bending, twisting, extension, colling) is predictable for a given external loading and internal pressure history. The basic objectives are to develop theorles for the motion and control of selected single elements and multiple element systems (beams) to be used a light-weight, fast-acting manipulators.

To meet the objectives, the first year of this three-year project was focused on three separate yet related top1cs. The first involved studies of an elephant's trunk from the viewpoint of a compliant, continuously flexible manipulator. The relationships among the load-carry1ng capacity, material (or muscle) properties and trunk geometry were investigated and are reported in Chapter I. Using clues from this animal study, particularly the observations of how a trunk colls around an object and then lifts 1t, the second study Involving the nonlinear mechanics of bending and colling tube-type element systems was carrled out. Those results are reported in Chapter II. The third study employed the linear theory of elasticity to predict the load and deformation behavior of cylindrical tubes with orthotrophy in the form of constant angle helices. The quantative results given In Chapter III show that such a tube element can be used as a torsion actuator, or a manipulator that twists about its longitudinal axis when pressurized. 


\section{ACKNOWLEDGEMENTS}

Thanks are due to several people who enthusiastically contributed to the elephant studies In Chapter I. Umesh Mahajan assisted in the analysis and interpretation of the data. Stephen A. Wainwright planned and carried out the experiments on a 11 ve elephant at the National Zoo in Washington, D.C. Lisa J. Croner assisted in these experiments and also did the laboratory studies on trunk muscle structure. Personnel at the Harvard Uni versity Museum of Comparative Zoology gave us access to source materlal. Edwin Gould, Bess Frank, Kathy Wallace, and Jim Jones at the National Zoo helped make the experiments possible.

Umesh Mahajan did the theoretical studies in Chapter II that involved the mechanics of the continuous manipulator. Th1s study comprises his Master's thesis for wh1ch James F. W11son was the advisor.

Gary OrgI11 and James F. Wilson did the analysis and calculations for the mechanical behavior of the orthotropic cylinders, the topic of Chapter III.

During all of these studies, H1lliam E. Isler, the project monitor, gave much support and encouragement.

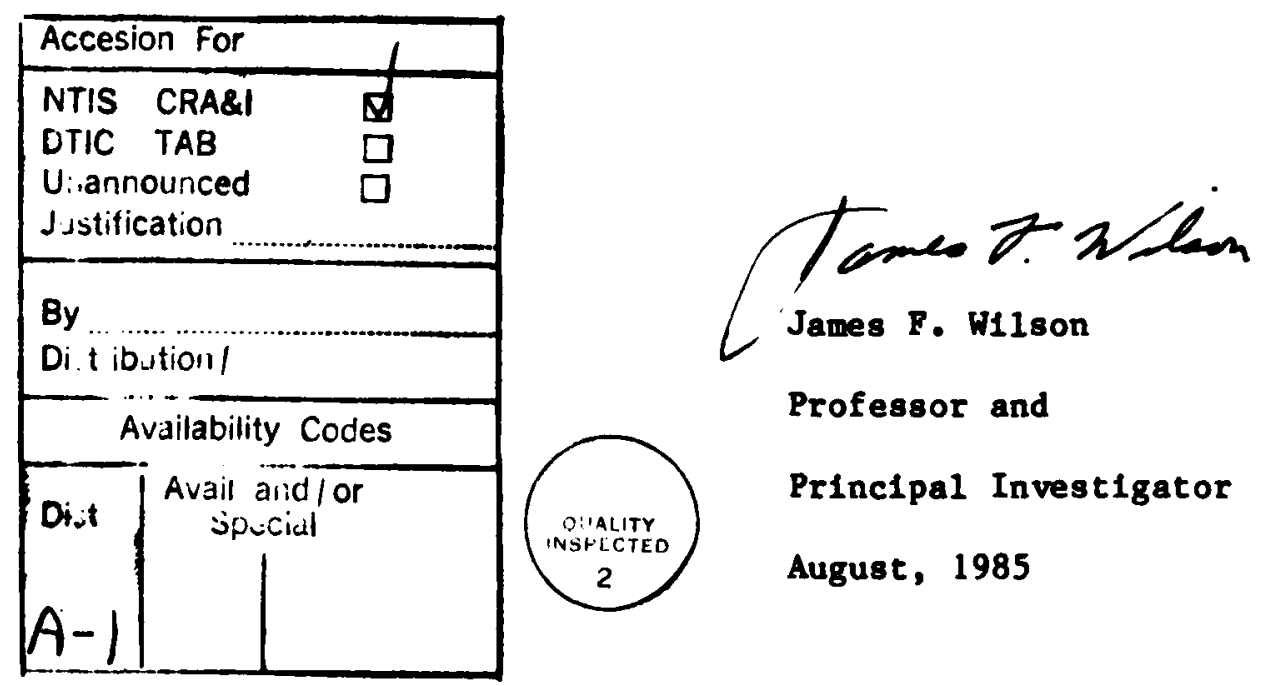

ii 
TABLE OF CONTENTS

SUMMARY

ACKNOWLEDGEMENTS

I. MECHANICS OF AN ELEPHANT TRUNK: THEORY AND MEASUREMENTS

Abstract

Introduction

Muscle Morphology

Lifting Experiments

Mathematical Model and Analysis

Numerical Results and Discussion

Nomenclature

References

MADE OF A NONLINEAR, COMPOSITE MATERIAL

Abstract $\quad 28$

Introduction 29

Analysis of a Typical Element $\quad 30$

Analysis of Motion and Load-Carrying

Capacity of a Continuous Manipulator Arm 37

Implementation of the Learning Concept 50

Numerical Examples and Discussion 56

References

III. LINEAR ANALYSIS OF UNIFORMLY STRESSED, ORTHOTROPIC CYLINDRICAL SHELLS

Abstract

Introduction

Constitutive Relationships

70

Evaluation of Material Constants $\quad 74$

Equilibrium and Compatibllity 78

Strain-Displacement Relations 79

Parametric Studies $\quad 80$

Design Examples 94

Summary and Conclusions 96

Nomenclature $\quad 98$

$\begin{array}{lr}\text { References } & 100\end{array}$

$\begin{array}{ll}\text { APPENDIX A: Computation of Neutral Axis } & 101\end{array}$

APPENDIX B: Program Listings $\quad 106$

$\begin{array}{ll}\text { Program Typel } & 106\end{array}$

$\begin{array}{ll}\text { Program Learn } & 108\end{array}$ 
I. MECHANICS OF AN ELEPHANT TRUNK:

THEORY AND MEASUREMENTS

\section{ABSTRACT}

Elephant trunks are versatile and strong. Their mechanical design may therefore be used as a source of ideas for the design of versatile, strong robotic manipulators. In this paper a mathematical model of an elephant trunk lifting a weight is developed based on assumptions parallel to those used in the analysis of prestressed, linear, composite beams. Data on overall trunk geometry obtained during weight-lifting and data for the trunk muscie distribution at a cross section are used to calculate an apparent tangent modulus for the trunk tissue. During a change in trunk curvature for which the uniform component of longitudinal prestrain remains a constant 30 percent, a typical value for the apparent modulus is of the order of $10^{6} \mathrm{~N} / \mathrm{m}^{2}$. This study represents an initial step toward more refined analyses that rationally relate the loading, the overall trunk geometry and the muscle structure while satisfying equilibrium conditions. 


\section{INTRODUCTION}

The trunk of an elephant is a tapering muscular structure, completely lacking rigid materials, of nearly circular cross-section. It is versatile and strong; trunks can shorten, extend, bend, and twist, and elephants have been trained to lift and manipulate masses of up to $300 \mathrm{~kg}$ with their trunks. This study is part of a larger study exploring the mechanics of trunk motion and strength, and aimed at discovering principles of trunk design that may be used in the design of similar, continuously flexible, versatile, strong robotic manipulators. A further discussion of animal muscular hydrostats and robotic mechanics is given by Wilson (1984).

The purpose of the present study is to correlate original data for the overall geometry of a trunk lifting a load with the reactive forces of the trunk muscles, to measure the average longitudinal strain of a trunk lifting a weight, and then to calculate an apparent modulus of trunk tissue. The apparent modulus is defined as the average tangent modulus for all of the longitudinal and oblique muscle tissue at a given cross section. This average tangent modulus $E_{a}$ represents the slope of the stress-strain curve in a uniaxial test in which this whole muscle group has a uniform longitudinal prestrain $\varepsilon_{w}$. The method used to calculate the modulus is one that does not require dissection of the animal involved, and may be used by zoologists interested in the apparent modulus of other animal appendages. This modulus calculated for the trunk may be used as a guide in choosing a material for trunk-like robotic manipulators.

The size and distribution of the trunk's four basic muscle masses--the radial, the longltudinal, and the two oblique layers--probably account for the elephant's ability to grasp and manipulate loads with its trunk. Although the role of each muscle type in trunk movement and load manipulation is not fully 
understood, it is probable that one can draw parallels to the mechanical behavior of squid tentacles and vertebrate tongues as outlined by Kier (1982). In this case contraction of selected oblique muscles would lead to torsion of the trunk about the longitudinal axis, uniform contraction of radial muscles would lead to uniform trunk lengthening, and contraction of longitudinal and radial muscles, acting separately or together on one side of the trunk, would lead to trunk bending.

In this analysis the longitudinal trunk loads (self-weight and payload) are assumed to be supported solely by the tensions of the longitudinal and oblique muscles. The corresponding longitudinal strain has two components: a relatively larger, uniform, contraction prestrain lobserved to be 20 to 35 percent before and during lifting); and a relatively small strain (two percent maximum) due to trunk bending and curvature--as the payload is lifted. In achieving the uniform contraction prestrain, the stress-strain behavior is probably very nonlinear. The tangent modulus (the slope of the stress-strain curve) may increase dramatically since muscles are much stiffer after contraction (Yamada and Evans, 1970). For the small strains associated only with trunk bending and small changes in trunk curvature between two states of lifting the payload, the analysis is based on classical beam theory. That is, the trunk is assumed to be composed of a linear, composite material (the longitudinal and oblique muscles) for which the apparent modulus on the side of the neutral axis under the least muscle tension is the same as the apparent modulus on the other side under the most muscle tension. The apparent modulus of this analysis, then, represents the average value of the tangent modulus for the muscles involved in incremental bending, given a uniform prestrain. The analysis implies that during incremental bending there is no change in chemical structure of the material with load other than the strains imposed on 
the chemical bonds by the load. Clearly, the trunk of a live elephant may not exactly satisfy all of the assumptions and conditions. However, local variations in the modulus of the components of muscle cells and fibrous connective tissues, and other local variations such as possible shifts in the neutral bending axis and possible nonlinear bending strain distributions, would have little effect on either the average apparent modulus for the load-carrying tissue acting as a group, or on the overall mechanical performance of the trunk. The approach herein represents the first step towards a more refined and accurate analysis of the trunk.

Briefly, the analysis and measurements proceed as follows. As described in the Mathematical Model and Analysis section, a linear strain distribution at a cross section and force equilibrium were used to calculate the location of the neutral axis of the trunk. To do this the cross-sectional areas of muscles were obtained from a drawing of a trunk cross-section and a discussion of detailed trunk morphology presented in the classical study of the elephant's head by Boas and Paulli (1908). The drawing of the cross-section was then estimated to coincide with a particular location along the length of the trunk. At this location the radij of curvature $p_{0}$ and $p_{1}$ at two lift positions were measured from films we took of an elephant lifting a known weight with its trunk. The second moment of area of the muscles, I, was determined from the size and distribution of the muscles in the Boas and Paulli cross-section. Based on moment equilibrium, $P_{0}, P_{1}$, and I were then used, as described below, to calculate the apparent modulus of trunk tissue.

This paper is divided into four parts. In the first two, the Muscle Morphology section and the Lifting Experiments section, we describe the sources of our measured data. We develop our mechanical model of the trunk in the Mathematical Model and Analysis section. We then present our calculated data in the Numerical Results and Discussion section. 


\section{MUSCLE MORPHOLOGY}

Figure 1 presents a schematic drawing adapted from Boas and Paulli (1908) showing the placement and orientation of the four basic muscle masses and the two main tendinous masses in a trunk of Elephas maximus, the Asian elephant. Longitudinal sheets of radial muscles radiate from the connective tissue around the two nostrils. These muscles insert dorsally and laterally into tendinous leaves, not shown in this figure, which in turn radiate and connect to a long tendinous sheet, which is shown, covering the dorsal and lateral muscle of the trunk. The outer insertion of the ventral radial muscles is not known. Longitudinal muscles run in the channels formed dorsally and laterally. by the radiating muscles. Some of the longitudinal muscles insert into the radiating tendons attached to the radiating muscles, and some end in the center of the trunk where their insertion is less obvious.

Two layers of oblique muscle, oriented in opposite directions and one interior to the other, run down the ventral side of the trunk. Both of these muscle masses are symnetrical about the sagittal plane. The outer layer inserts ventrally along the sides of a long ventral tendon and runs laterally to insert along the sides of the dorso-lateral tendinous sheet. The inner layer inserts ventrally into the inner portion of the ventral tendon, and the lateral insertion of this muscle mass is not known. The orientation of the oblique muscles changes down the length of the trunk. Proximally the outer layer is almost transverse in orientation, but it gradually changes so that distally the fibers run almost longitudinally. The inner layer is exactly the reverse. Proximally, the inner oblique muscle fibers run almost longitudinally and distally they run almost transversely. Both the dorso-lateral and ventral tendons are separated proximally from the skin by a layer of loose connective tissue; further down the trunk they are more intimately connected to the skin. 


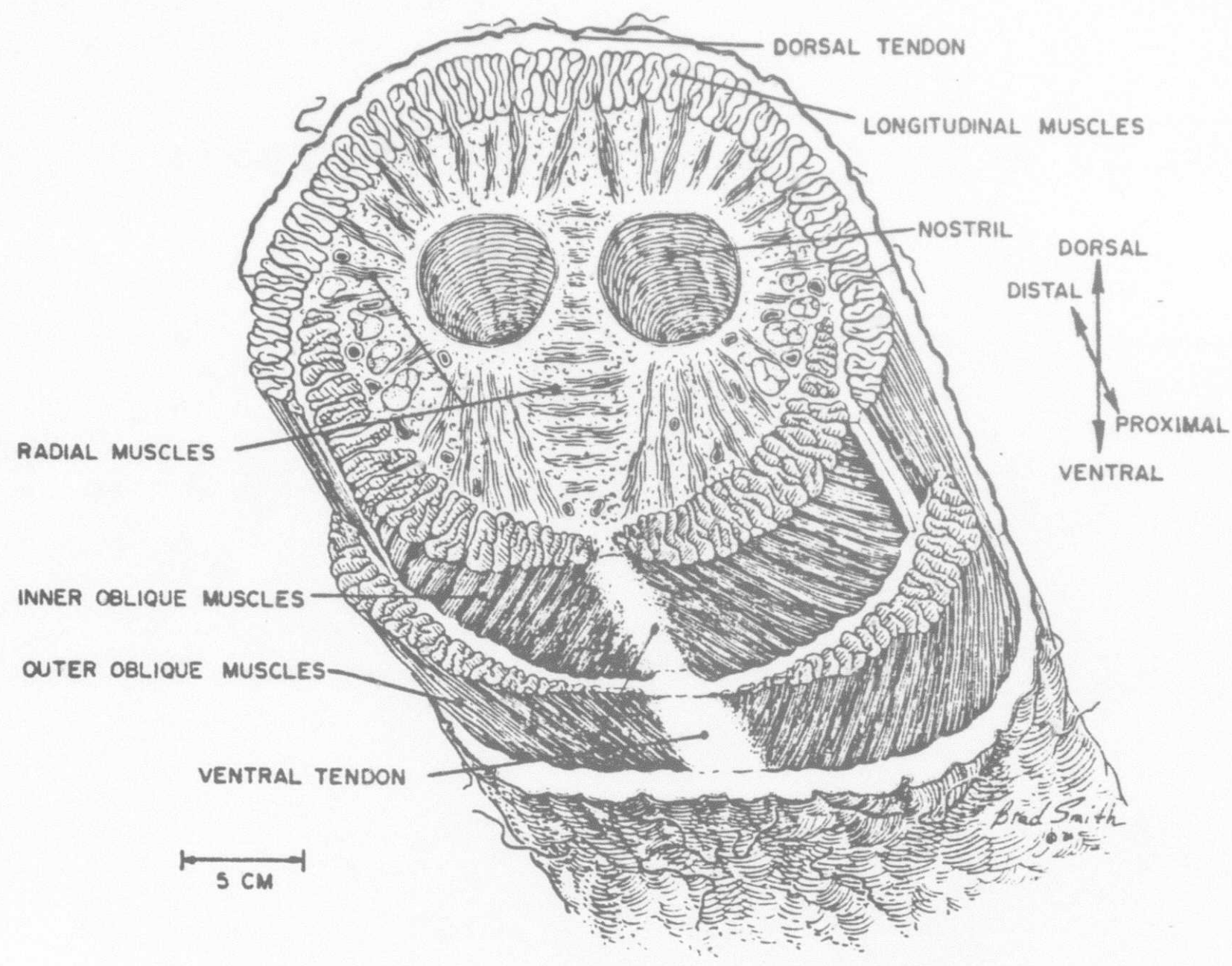

Figure 1 Cross section of an elephant's trunk showing the distribution of muscles (based on information taken from Boas and Paulli (1908) by Brad Smith) 
We took $16 \mathrm{~mm}$ movies of two mature Asian elephants at the National 200 in Washington, D.C. This involved filming the trunks while the elephants lifted payloads at the tips of their trunks. These elephants had trunks about $1.5 \mathrm{~m}$ long when in a fully extended, relaxed state. The nominal outside diameters of these trunks were about $32 \mathrm{~cm}$ at the head and $8 \mathrm{~cm}$ at the tip.

For our analysis, we used both data from films and data obtained from drawings of cross-sections of an elephant's trunk from the Boas and Paulli treatise. A composite of such cross-sections is shown in Figure 1. Based on the position of radial muscles below the nostrils, the shape of the nostrils, and the relative thickness of the oblique muscles in cross-section, we deduced that this particular transverse cross-section was located at position A-A along the trunk as shown in scale drawings of photographs, Figures 2. The length scales shown in Figures 1 and 2 are those for the Asian elephant that we filmed. The important assumption we used in deriving the particular numerical results that follow is that the muscle mass distribution for section $A-A$ in Figure 2 is geometrically similar to that of Figure 1.

\section{LIFTING EXPERIMENTS}

In preparation for a typical experiment involving a live Asian elephant, white zinc oxide spots were first painted along the midlateral line on the side of the trunk, as shown in Figure 2. Each white spot was located along the trunk at a distance $\ell_{j}$ from the spot nearest the head. The trunk was photographed in what appeared to be its longest and fully relaxed state, in a near vertical position.

The sequence of events involving the elephant lifting a payload with the tip of its trunk was recorded using a Canon Scopic $16 \mathrm{~mm}$ movie camera situated about $12 \mathrm{~m}$ directly to the side of the animal. Trunk motion was in the 

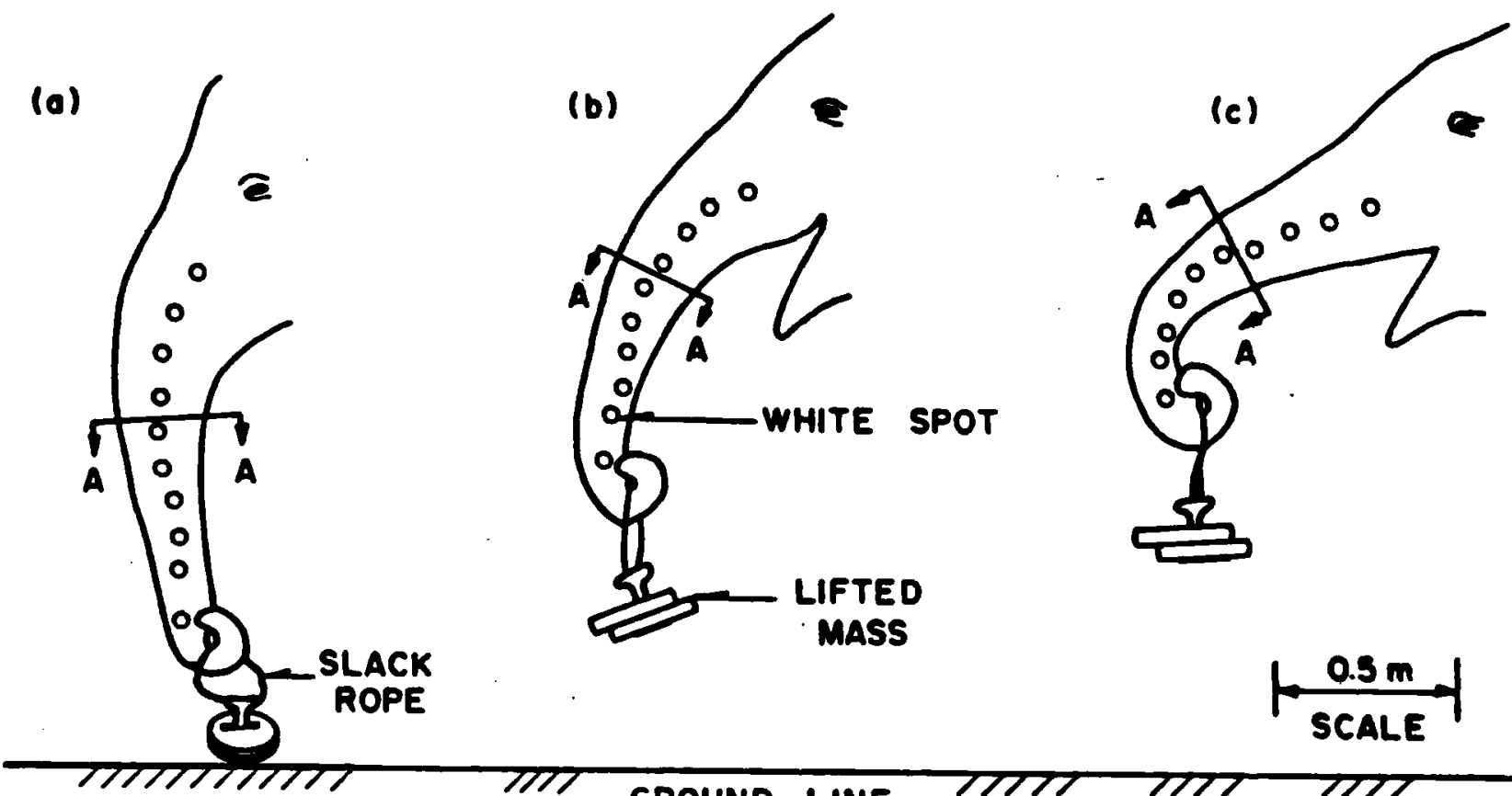

LIFTED
MASS

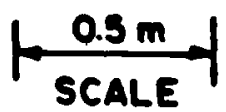

GROUND LINE

Figure 2 Trunk contracted with payload on ground (a); payload at an intermediate position (b); and payload at its highest position (c). Traced from projected movie images. 
vertical plane, parallel to the side of the animal and perpendicular to the plane of movie images. The sequence of events was as follows.

1. The trunk tip looped around the rope attached to a payload mass of $26.2 \mathrm{~kg}$. With the payload still on the ground, the entire trunk shortened and thickened before lifting, resulting in the configuration of Figure $2 a$. The average longitudinal contracted strain $\varepsilon_{u}$ was deduced by comparing the locations of the trunk spots in the relaxed and contracted states. That is, each spot moved from its relaxed position $\ell_{i}$ by an amount $\Delta \ell_{i}$ as depicted by the insert of Figure 3. The results are plotted as circles in Figure 3, from which the slope or initial strain before lifting was deduced by a least squares straight line fit as $\varepsilon_{u}=-24.4 \%$, a nearly uniform value along the trunk.

2. The coiled trunk tip stretched a bit while the elephant lifted its head and dragged the payload along the ground.

3. The payload was lifted by an upward motion of the head, accompanied by a further shortening of the trunk, and a further curling motion of the whole trunk. Figures $2 \mathrm{~b}$ and $2 \mathrm{c}$ depict the trunk with the payload lifted to an intermediate and the nighest position. The average value of $\varepsilon_{u}$ for these last two positions based on the data of Figure 3 were $-31.5 \%$ and $-33.0 \%$ respectively.

A careful study of enlarged prints from the film showed that the radius of curvature $\rho$ traced by the white dots in the plane of motion changed significantly. The values of $p$ and the corresponding uncertainties in measurement \pm $\Delta \rho$ at section $A-A$ of the trunk were measured as $p_{0}=1.18 \mathrm{~m} \pm 0.06 \mathrm{~m}$ and $p_{1}=$ $1.67 \mathrm{~m} \pm 0.25 \mathrm{~m}$, corresponding respectively to the intermediate and highest positions of the trunk shown in Figure 2. The analysis that follows relates these bending strains to the radius of curvature. 
The purpose of this section is twofold: to define a rational, static. mathematical model of the trunk muscle system that supports both the trunk load and payload and to calculate the apparent elastic modulus of the trunk at section $A-A$ when the trunk is between the two positions shown in Figure $2 b$ and 2c. A few simplifying assumptions are needed. First, the longitudinal strain $\varepsilon_{x}$ due only to trunk curvature $p$ has a linear distribution from dorsal to ventral. That is

$$
\varepsilon_{x}=-\frac{y}{p}
$$

where Figure 4 depicts this strain at position $y$ from the neutral axis of bending. This linear strain distribution superimposed on the uniform contractual strain $\varepsilon_{u}$ from muscle pretension gives the total strain at the trunk cross section. Second, in comparison to this total strain distribution, it is reasonable to assume that $\varepsilon_{y} \approx \gamma_{x y}=0$, where $\varepsilon_{y}$ is the normal strain along the $y$ direction and $\gamma_{x y}$ is the transverse shear strain due to changes in the bending moment and curvature along the trunk. These assumptions, which are consistent with elementary beam theory, lead to an expression for the normal strain $\varepsilon_{\theta}$ along the length of each oblique muscle inclined at an acute angle $\theta$ with the longitudinal axis (Figure 3 and 4) or

$$
\varepsilon_{\theta}=\varepsilon_{x} \cos ^{2} \theta
$$

Equation (2) follows from the strain transformation equations derived, for instance, by Timoshenko and Goodier (1951), where $\varepsilon_{y}$ and $\gamma_{x y}$ are zero.

The third assumption is that the longitudinal tensile stress in each muscle mass is linear with its longitudinal contracted strain, or

$$
\sigma_{x}=-E_{a} \varepsilon_{x} ; \quad \sigma_{\theta}=-E_{a} \varepsilon_{\theta}
$$

where $\sigma_{x}$ and $\sigma_{\theta}$ are the normal stresses for the longitudinal muscies and 
oblique muscles, respectively. Here, $E_{a}$ is the apparent elastic modulus of the muscle mass, which is assumed to be the same for each muscle type.

The fourth assumption is that only the muscles carry the stresses set up by the trunk's own mass and by the payload. Thus, the tissue surrounding the muscles, although following the linear strain distribution, has a negligible stiffness compared to the stiffness of the activated muscles. A consequence of these assumptions is that the neutral axis in bending does not shift for small changes in trunk curvatures. The accuracy of these assumptions is not known.

At a typical cross section such as $A-A$ in Figure 4 , the condition of force equilibrium in the $x$ direction, or perpendicular to this transverse section, must be met. This equilibrium condition establishes the location of the neutral axis $y=0$, or the plane along which the muscle strain due to trunk curvature alone is always zero. Force equilibrium is expressed as:

$$
\int_{A_{x}} \sigma_{x} d A_{x}+\int_{A_{0}} \sigma_{\theta} \cos ^{2} \theta d A_{0}=0
$$

where $A_{x}$ is the total transverse cross sectional area for the longitudinal muscles and $A_{0}$ is the total observed cross sectional area of the oblique muscles projected on the same transverse cross section (the $x=0$ plane). From Figure 5, it is observed that for the oblique muscles the cross section area element normal to $\sigma_{\theta}$ is $d A_{0} \cos \theta$, that the corresponding force is $\sigma_{\theta} \cos \theta d A_{0}$, and the component of this force projected along $x$ is thus $\sigma_{\theta} \cos ^{2} \theta d A_{0}$, which leads to the second integral on the left in Equation (4). With Equations (1), (2) and (3), Equation (4) is rewritten as

$$
\int_{A_{X}} y d A_{X}+\int_{A_{0}} y \cos ^{4} \theta d A_{0}=0
$$

A practical way to locate the neutral axis is as follows. As shown in Figure 4, the neutral axis at $y=0$ is located at a distance $\bar{z}$ from a datum 

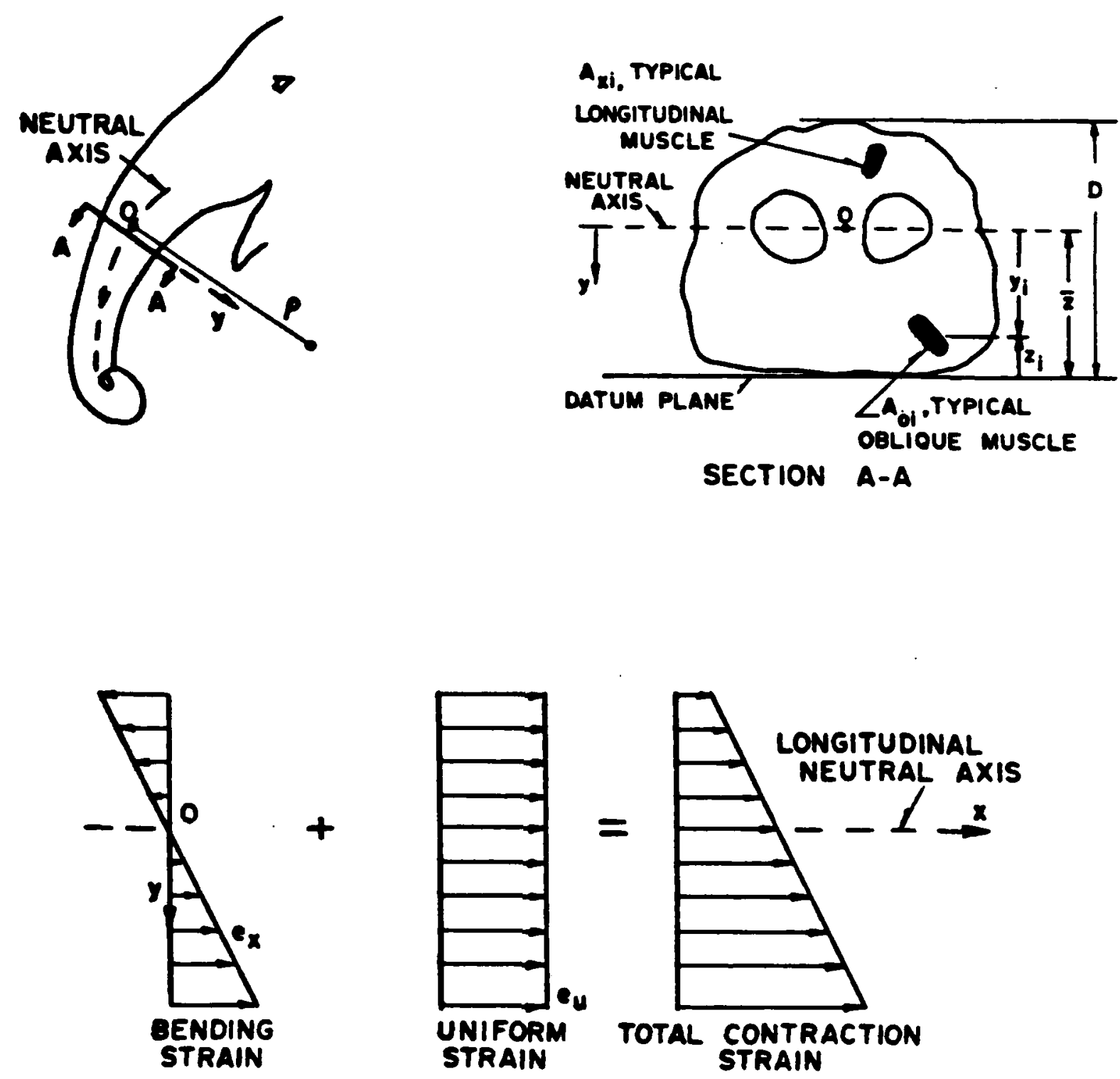

Figure 4. Definitions of trunk geometry and longitudinal strain. 


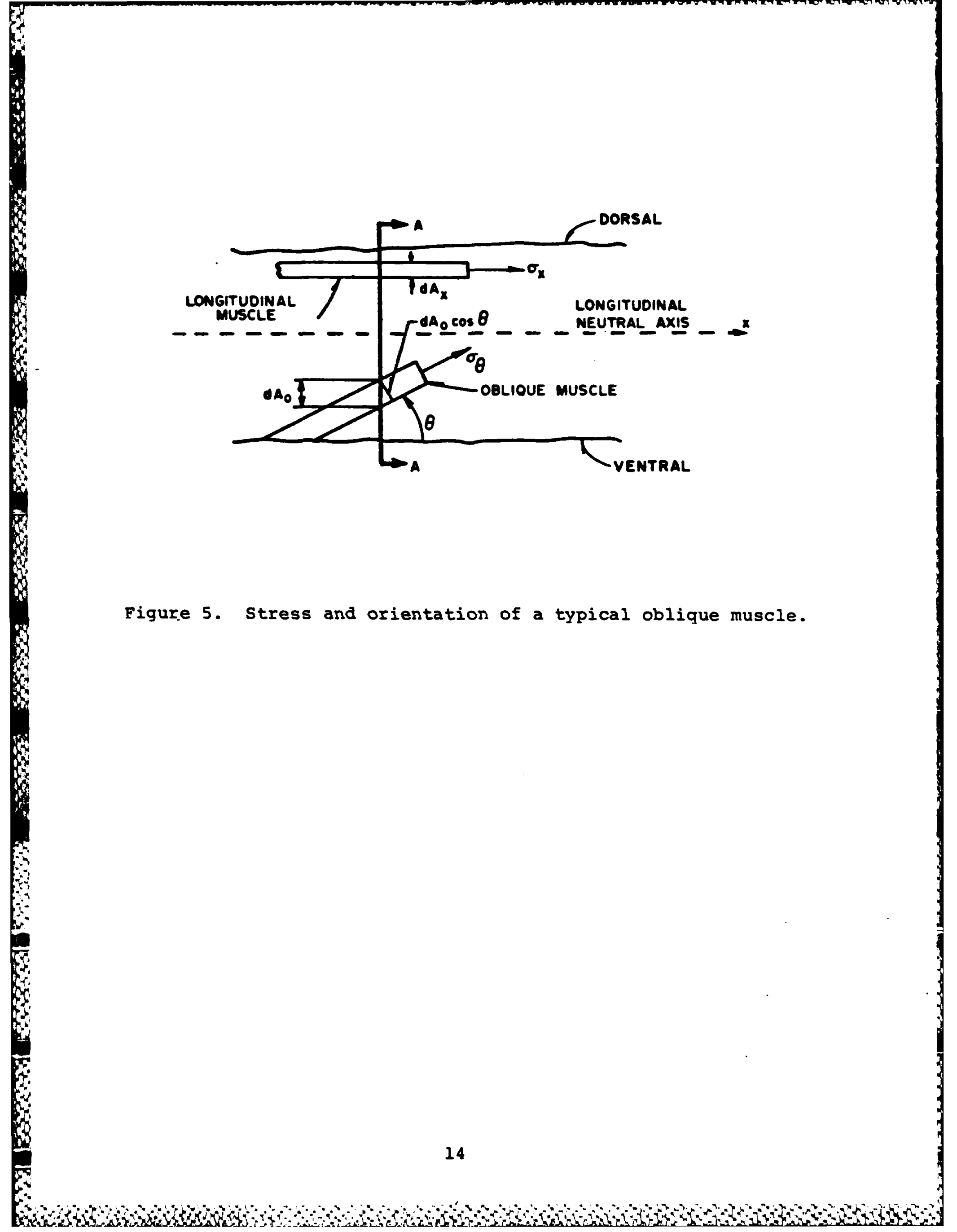


axis chosen as the ventral edge. If the centroid of the area for each muscle $i$ of area $A_{x i}$ or $A_{0 i}$ is located at $y=y_{i}$, then the centroidal distance $z_{j}$ from the datum axis is defined as

$$
z_{i}=\bar{z}-y_{i}
$$

When Equation (6) is combined with Equation (5), with the differential areas replaced by the areas $A_{x i}$ and $A_{0 i}$, and the integrals replaced by finite sums, the result is a practical formula for locating the neutral axis. That is

$$
z=\frac{\sum_{i=1}^{N} z_{i} A_{x i}+\sum_{i=1}^{M} z_{i} A_{0 i} \cos ^{4} \theta_{i}}{\sum_{i=1}^{N} A_{x i}+\sum_{i=1}^{M} A_{0 i} \cos ^{4} \theta_{i}}
$$

where there are $N$ longitudinal muscles and $M$ oblique muscles at a cross section. Care must be taken to use the correct angles for the oblique muscle areas. For instance, for section $A-A$ of Figure $5, \theta=\theta_{i}=29.1$ deg for the inner oblique muscles and $\theta=\theta_{i}=34.1 \mathrm{deg}$ for the outer oblique muscles.

Having located the neutral axis, the bending strain distribution can be calculated from Equation (1) if the radius of curvature is known. Of interest here is the change in the bending strain distribution between two loading states at section A-A. For instance, the difference in longitudinal muscle strain $\Delta \varepsilon_{X}$ for a trunk configuration in its highest position (Figure 2c) for $\rho=\rho_{1}$ and in its intermediate position (Figure $2 b$ ) for $\rho=\rho_{0}$ is

$$
\Delta \varepsilon_{x}=-\frac{y}{p_{1}}-\left(-\frac{y}{\rho_{0}}\right)=-\frac{y}{p}
$$

where

$$
\frac{1}{p}=\frac{p_{0}-P_{1}}{P_{0} P_{1}}
$$

At a fixed location $y$, the change in strain $\Delta \varepsilon_{\theta}$ in a typical oblique muscle will always be less than $\Delta \varepsilon_{x}$, or from Equation (2) 


$$
\Delta \varepsilon_{\theta}=-\frac{y}{\bar{\rho}} \cos ^{2} \theta
$$

Accompanying these strain changes $\Delta \varepsilon_{X}$ and $\Delta \varepsilon_{\theta}$ during lifting are corresponding changes in the normal stress distributions, $\Delta \sigma_{x}$ and $\Delta \sigma_{\theta}$. These are the muscle reactions in response to shifts in two types of external loads causing moments at section A-A. One of these loads is due to the trunk mass segment $m_{t}$ (below section $A-A$ to the tip) which causes a differential moment of $m_{t} g\left(d_{1}-d_{0}\right)$. The other load is due to the payload mass $m_{p}$ which causes a differential moment $m_{p} g\left(e_{1}-e_{0}\right)$. The load offset distances $d_{0}, d_{1}, e_{0}$ and $e_{1}$ are defined in the free body sketches of Figure 6. For moment equilibrium at Section $A-A$, it follows that

$$
m_{t} g\left(d_{1}-d_{0}\right)+m_{p} g\left(e_{1}-e_{0}\right)=\int_{A_{x}} y \Delta \sigma_{x} d A_{x}+\int_{A_{0}} y \Delta \sigma_{\theta} \cos ^{2} \theta d A_{0}
$$

Here $\Delta \sigma_{x} d A_{x}$ and $\Delta \sigma_{\theta} \cos ^{2} \theta d A_{0}$ are the changes of the $x$-directed forces in the longitudinal and oblique muscles, respectively, which, when multiplied by their moment arms $y$ and integrated over section A-A, leads to Equation (11). Invoking the linear relationships of Equations (3), along with Equations (2), (8), and (10), the differential stresses become

$$
\begin{array}{r}
\Delta \sigma_{x}=-E_{a} \Delta \varepsilon_{x}=E_{a} \frac{y}{\bar{p}} \\
\Delta \sigma_{\theta}=E_{a} \Delta \varepsilon_{\theta}=E_{a} \frac{y}{p} \cos ^{2} \theta
\end{array}
$$

With Equations (12) and (13), Equation (11) can be solved for the apparent modulus, or

$$
E_{a}=\frac{\bar{p}}{I}\left[m_{t} g\left(d_{1}-d_{0}\right)+m_{p} g\left(e_{1}-e_{0}\right)\right]
$$

where $\bar{\rho}$ is given by Equation (9) and I, the second moment of the muscle area with respect to the neutral axis $(y=0)$, is 


$$
I=\int_{A_{x}} y^{2} d A_{x}+\int_{A_{0}} y^{2} \cos ^{4} \theta d A_{0}
$$

With a knowledge of the muscle morphology as shown in Figure 1, along with trunk curvatures and the loading terms, the apparent modulus $E_{a}$ may be calculated from Equations (14) and (15). Then the differential stress and strain distributions due only to a curvature change may be calculated from Equations (12) and (13). The appropriate measurements and typical numerical results are now discussed.

\section{NUMERICAL RESULTS AND DISCUSSION}

To obtain the desired numerical results, a clear and properly scaled outline of the longitudinal and oblique muscle areas at Section A-A is needed. Such a drawing, deduced from the Boas and Paulli treatise (1908) is shown in Figure 7. This muscle distribution and the measured data listed in Table 1 were used to calculate the apparent trunk modulus. The geometry of figure 7 was scaled to match the cross section $A-A$ of our subject animal's trunk, for which the section height $D$ (Figure 4) was measured from photographs as $24 \mathrm{~cm}$. of the 150 muscle areas shown in figure 7, 78 are longitudinal, 51 are inner oblique $(\theta=29.1 \mathrm{deg})$, and 21 are outer oblique $(\theta=34.1 \mathrm{deg})$. Using a fine grid overlay, each muscle area or projected area and its respective centroidal distance to the datum plane was calculated. The total areas $A_{x}$ and $A_{0}$ are given in Table 2. With the values of $\theta$ measured from drawings in the Boas and Paulli treatise (1908), $\bar{z}$ was calculated from Equation (7) as $12.0 \mathrm{~cm}$. Since $D=24 \mathrm{~cm}$, the neutral axis lies at the mid-height of this cross section. The value of I was then calculated by numerically integrating Equation (15), yielding $1=4270 \mathrm{~cm}^{4}$. Of course, these numerical results, although precisely calculated to three significant figures, are only as accurate as the artist's original drawings of the muscle distributions. 


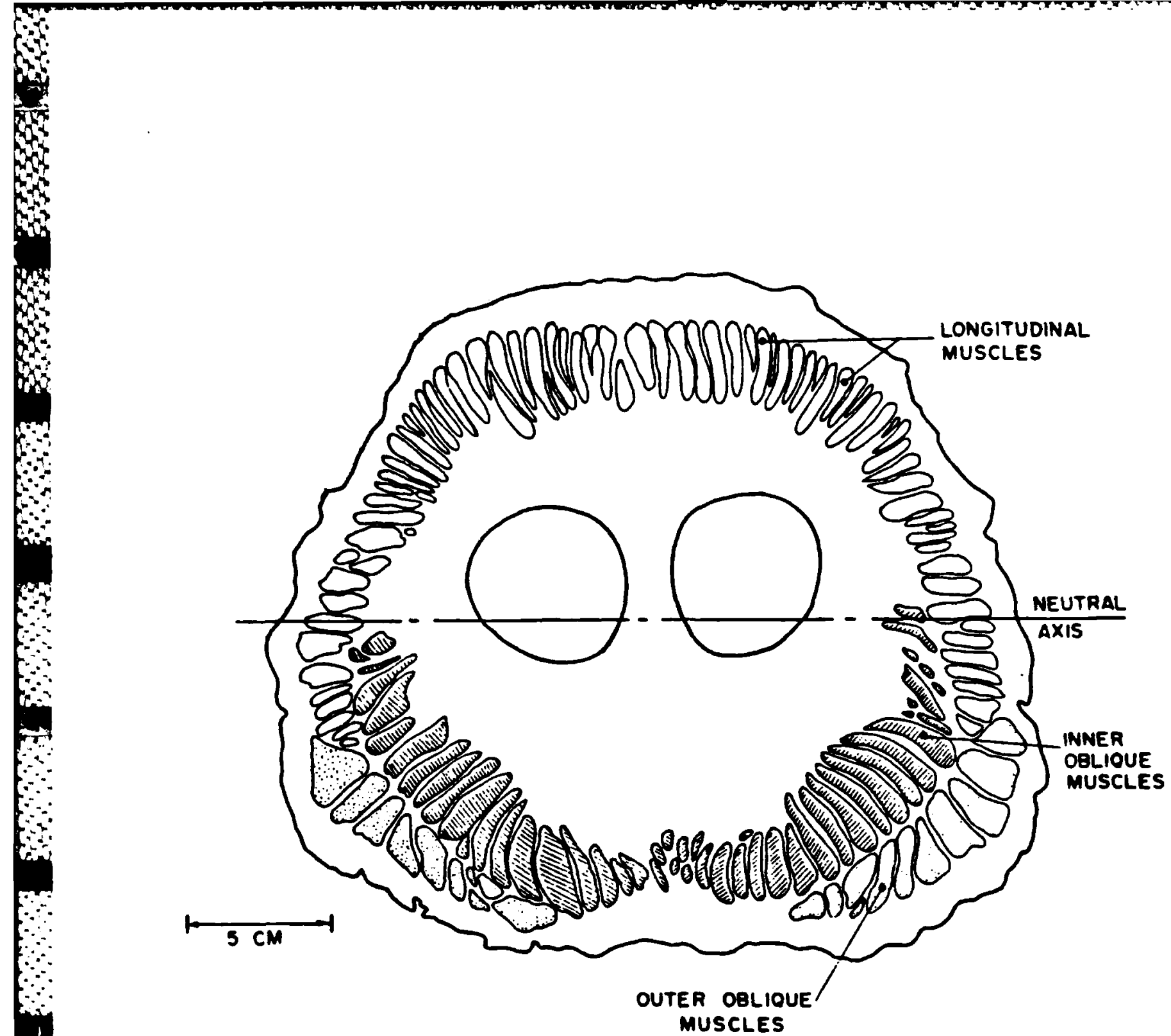

Figure 7. Idealized muscle distribution used for calculations (adapted from Boas and Paulli, 1908). 
Table 1 - Summary of Measured Data

Symbol and Value

$D=24 \mathrm{~cm}$

$d_{0}=5.0 \mathrm{~cm}$

$d_{1}=8.0 \mathrm{~cm}$

$e_{0}=9.29 \mathrm{~cm}$

$e_{1}=13.27 \mathrm{~cm}$

$\ell_{j}, \Delta \ell_{j}$

$m_{p}=26.2 \mathrm{~kg}$

$m_{t}=16.37 \mathrm{~kg}$

$M=78$

$N=72$

$r=1180 \mathrm{~kg} / \mathrm{m}^{3}$

$\theta=29.1 \mathrm{deg}$

$\theta=34.1 \mathrm{deg}$

$p_{0}=118$

$p_{1}=167$

$\Delta \rho_{0}= \pm 6 \mathrm{~cm}$

$\Delta p_{1}= \pm 25 \mathrm{~cm}$
Meaning

trunk height (y direction) at section $A-A$

offset distance of $m_{t}$ (intermediate), Fig. 6a

offset distance of $m_{t}$ (highest), Fig. 6b

offset distance of $m_{p}$ (intermediate), Fig. $6 a$

offset distance of $m_{p}$ (highest), Fig. $6 b$

spot locations, plotted in Fig. 3

mass of payload

mass of trunk from tip to section $A-A$

number of longitudinal muscles at section $A-A$

number of oblique muscles at section $A-A$

mass density of trunk

inclination of inner oblique muscles, Fig. 5

inclination of outer oblique muscles, Fig. 5

radius of curvature of section $A-A, F i g$. $6 A$

radius of curvature at section $A-A, F i g .6 b$

uncertainty of $\rho_{0}$ measurement

uncertainty of $\rho_{1}$ measurement 
Table 2 - Summary of Calculated Data

\begin{tabular}{|c|c|c|}
\hline $\begin{array}{l}\text { Symbol } \\
\text { and Value }\end{array}$ & Meaning & $\begin{array}{l}\text { Equation } \\
\text { or Figure }\end{array}$ \\
\hline$=54.6 \mathrm{~cm}^{2}$ & $\begin{array}{l}\text { area of all longitudinal muscles on } \\
x=0 \text { plane }\end{array}$ & Fig. 7 \\
\hline$A_{0}=185.7 \mathrm{~cm}^{2}$ & $\begin{array}{l}\text { projected area of all oblique muscles on } \\
x=0 \text { plane (inner oblique: } 160 \mathrm{~cm}^{2} \text {; outer } \\
\text { oblique: } 25.7 \mathrm{~cm}^{2} \text { ) }\end{array}$ & Fig. 7 \\
\hline$E_{a}=1.33 \times 10^{6} \mathrm{~N} / \mathrm{m}^{2}$ & apparent modulus of trunk & Eq. (14) \\
\hline$\Delta E_{a}= \pm 0.532 \times 10^{6} \mathrm{~N} / \mathrm{m}^{2}$ & uncertainty in $E_{a}$ & Eq. (16) \\
\hline$I=4270 \mathrm{~cm}^{4}$ & area moment of all muscles & Eq. (15) \\
\hline $\bar{z}=12.0 \mathrm{~cm}$ & location of neutral axis & Fig. 4 \\
\hline$\Delta \sigma_{x}=3.22 \times 10^{4} \mathrm{~N} / \mathrm{m}^{2}$ & max. stress change, longitudinal muscle & Eq. (12) \\
\hline$\Delta \sigma_{\theta}=2.23 \times 10^{4} \mathrm{~N} / \mathrm{m}^{2}$ & max. stress change, inner oblique muscle & Eq. 13) \\
\hline$\Delta \sigma_{\theta}=2.37 \times 10^{4} \mathrm{~N} / \mathrm{m}^{2}$ & max. stress change, outer oblique muscle & Eq. (13) \\
\hline$\Delta \varepsilon_{x}=0.0242$ & max. strain change, longitudinal muscle & $E q \cdot(8)$ \\
\hline$\Delta \varepsilon_{\theta}=0.0168$ & max. strain change, inner oblique muscle & Eq. $(10)$ \\
\hline$\Delta \varepsilon_{\theta}=0.0178$ & max. strain change, outer oblique muscle & Eq. $(10)$ \\
\hline$\varepsilon_{u}=-0.244$ & uniform strain, lowest position & Fig. 3 \\
\hline$\varepsilon_{u}=-0.315$ & uniform strain, intermediate position & Fig. 3 \\
\hline$\varepsilon_{u}=-0.330$ & uniform strain, highest position & Fig. 3 \\
\hline & differential curvature & Eq. (9) \\
\hline
\end{tabular}


The external moments at section $A-A$ due to the payload and the trunk mass were measured for the trunk in two different positions: the intermediate and the nighest positions shown in Figures 2 and 6 . The reason for choosing these two positions was that the uniform component of longitudinal trunk strain was nearly the same for both positions ( $\varepsilon_{u}$ was 0.315 and 0.330 , respectively). Thus, the radii of curvature $p_{0}$ and $p_{1}$ as measured from photographs did not need to be corrected to account for changes in $\varepsilon_{u}$. The importance of highly accurate measures of these radii to an accurate calculation of the apparent modulus will be discussed below.

The quantities defining the differential moment between the selected trunk positions are enclosed in the square brackets of Equation (14). Measurements were made for each of these quantities. The payload mass $m_{p}$ was $26.2 \mathrm{~kg}$. The offset distances $e_{0}$ and $e_{1}$ for this load, as well as the volume seyment of the trunk from tip to section $A-A$, were deduced from photographs (see Figure 6 ). The centroidal distances $d_{1}$ and $d_{2}$ for this volume segment were calculated assuming that the volume was a series of right, truncated cones. The volumes of the two nostrils, deduced from the Boas and Paulli treatise, were treated as voids. The product of this volume segment and the trunk's mass density then gave the value $m_{t}=16.37 \mathrm{~kg}$. Our measurements of the mass density, made on selected samples from different areas of a dead elephant's trunk, yielded an average mass density of $1180 \mathrm{~kg} / \mathrm{m}^{3}$, with a maximum variation of 8 percent among samples.

The remaining quantity needed to calculate $E_{a}$ given by Equation (14) is the differential curvature $\dot{p}$. This is defined by Equation ( 9 ) in terms of the radil of curvatures $P_{0}$ and $P_{1}$ at section $A-A$. Accurate measures of these radii were more difficult to deduce from enlarged photographs than the data previously discussed. More closely spaced and more carefully aligned white 
spots along the trunk would lead to better measurements. In the present case, $P_{0}=118 \pm 6 \mathrm{~cm}$ and $P_{1}=167 \pm 25 \mathrm{~cm}$, showing an uncertainty of 5 percent and 15 percent, respectively. For the nominal values of the radii, Equation (9) gave $\bar{p}=-402 \mathrm{~cm}$.

Using the measured data discussed above and summarized in Table 1, the apparent modulus was calculated from Equation (14), or $E_{a}=1.33 \times 10^{6} \mathrm{~N} / \mathrm{m}^{2}$. It is interesting to note that this modulus compares favorably with the tangent modulus of human Sartorius muscle $\left(1.77 \times 10^{6} \mathrm{~N} / \mathrm{m}^{2}\right)$ and dog Rectus abdominis muscle $\left(4.0 \times 10^{6} \mathrm{~N} / \mathrm{m}^{2}\right)$ at prestrains of 60 to 65 percent, as estimated from data presented by Yamada and Evans (1970). Also, this modulus agrees within 10 percent with Young's modulus of a comercially available, synthetic rubber, Type $A 60 B^{*}$.

Further calculations lead to the differential strain distributions in the longitudinal muscles, $\Delta \varepsilon_{x}$, and in the oblique muscles, $\Delta \varepsilon_{\theta}$, and their corresponding differential stresses, $\Delta \sigma_{x}$ and $\Delta \sigma_{\theta}$. The maximum value for each of these quantities was calculated from Equations (8), (10), (12) and (13) based on the maximum distance $y$ extending from the neutral axis to the centroid of the most outlying muscle. The numerical results summarized in Table 2 reveal that the maximum differential muscle strains range from about 1.7 to 2.5 percent, and the maximum differential stresses range from about 2.2 $\times 10^{4}$ to $3.2 \times 10^{4} \mathrm{~N} / \mathrm{m}^{2}$. In fact, at any chosen distance $y$ from the neutral axis, calculations showed rather narrow ranges for the differential strains and differential-stresses among the three types of muscles. It is apparent that the muscles do a good job in sharing the load at this cross section. Given the validity of the mathematical model, for which the main assumptions were the linearity of strain over the cross section and the linear Thanufactured by The Lord Corporation, Erie, PA. 
stress-strain law, the accuracy of the numerical results were found to depend strongly on the accuracy of $\rho_{0}$ and $\rho_{1}$. These were the most uncertain of all of the measured parameters. The analysis of $\mathrm{Kl}$ ine and MCClintock (1953) may be used to show just how sensitive the calculation of $E_{a}$ is to the measured uncertainties $\Delta p_{0}$ and $\Delta p_{1}$ of $p_{0}$ and $p_{1}$, respectively. The corresponding uncertainty in $E_{a}$, or $\Delta E_{a}$ for these two parameters only is given by

$$
\Delta E_{a}=\left[\left(\frac{\partial E_{a}}{\partial p_{0}} \Delta p_{0}\right)^{2}+\left(\frac{\partial E_{a}}{\partial p_{1}} \Delta p_{1}\right)^{2}\right]^{1 / 2}
$$

When from Equation (9) is substituted into Equation (14) and the result is differentiated according to Equation (16), the result is

$$
\frac{\Delta E_{a}}{E a}=\frac{1}{p_{0} p_{1}\left|p_{0}-p_{1}\right|}\left(p_{1}^{4} \Delta p_{0}^{2}+p_{0}^{4} \Delta p_{1}{ }^{2}\right)^{1 / 2}
$$

With the nominal values of the radii and their deviations as listed in Table 1, the numerical value of $\Delta E_{a} / E_{a}$ calculated from Equation (17) is 0.40 . Thus, for relatively small uncertainties in radif (5 percent and 15 percent), the uncertainty in the apparent modulus is 40 percent, where $E_{a}=1.33 \times 10^{6} \mathrm{H} / \mathrm{m}^{2}$ $\pm 0.532 \times 10^{6} \mathrm{H} / \mathrm{m}^{2}$.

Further studies are now underway that involve microscopic studies of muscle morphology at different cross sections in a dead elephant's trunk, and also the direct measurements of tensile properties of connective tissue. As further data becomes avaflable, the present mathematical model may be refined. Thus, more exact connections may be deduced among the trunk's system parameters including its external loading, its overall shape during lifting, its muscle distribution, and the mechanical properties of its muscles. The results may provide clues to future mechanical designs of continuously flextble robotic manipulators made of polymeric materials. 


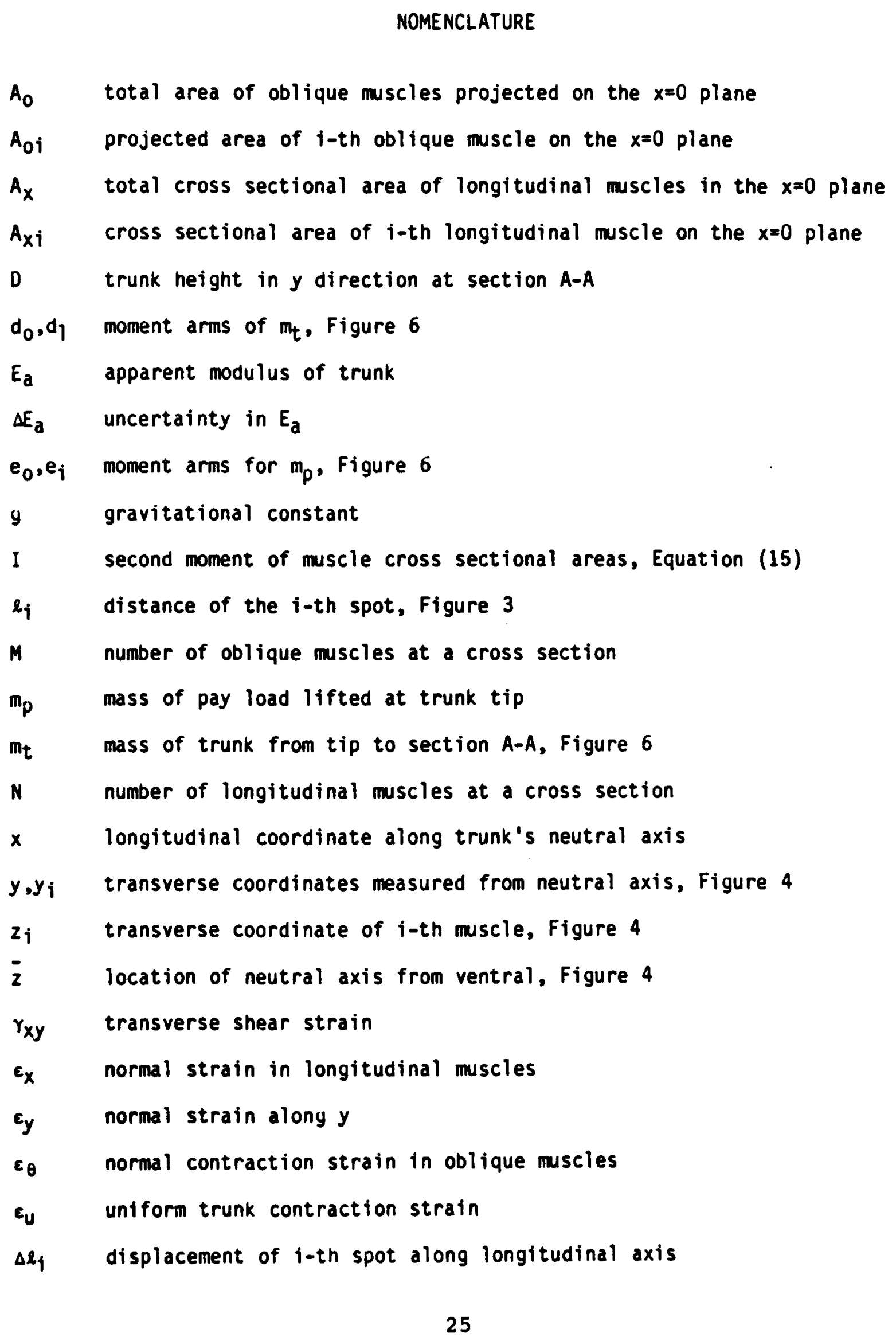




\section{REFERENCES}

Boas, J. E. V., and Paulli, S. (1908) The Elephant's Head: Studies in the Comparative Anatomy of the Head of the Indian Elephant and Other Mamnals, Part I, Gustav Fisher, Jena, 1-79.

Kier, W. M. (1982) The functional morpnology of the musculature of squid (10liginidae) arms and tentacles, Journal of Morphology, 12, 179-192.

Kline, S. J. and McClintock, F. A. (1953) Describing uncertainties in single-sample experiments, Mechanical Engineering, January, 3-11.

Timoshenko, S. and Goodier, J. N. (1951) Theory of Elasticity, McGraw-Hill, NY, 14-17.

Wilson, J. F. (1984) Robotic mechanics and animal morphology, Robotics and Artificial Intelligence, MATO ASI Series F1l, M. Brady, ed., Springer-Verlag, Berlin, 419-443.

Yamada, H. and Evans, F. G., editors (1970). Strength of Biological Materials, Williams and Wilkins, Baltimore, MD, 95-96. 
11. MECHANICS OF A CONTINUOUS MANIPULATOR MADE OF A NONL INEAR, COMPOSITE MATERIAL

\section{ABSTRACT}

This research deals with the analysis of light weight, flexible and fast moving robotic arms. A typical arm consisting of hollow cylindrical elements, bends and coils around a load and moves it to the desired position by varying the internal pressures in the individual elements. The theoretical derivations relating arm positions and loading incorporate nonlinear material properties and large deflections. A learning program is developed that stores information about arm motion, information that is used in subsequent manipulations to achieve efficient lifting scenarios. Typical numerical results for arm designs will be verified experimentally in the near future. 


\section{INTRODUCTION}

Robots are programmed machines designed to carry out desired tasks for humans $[1,2]$. Their use in the industry is rapidly increasing and extensive research is underway with the aim of improving their capabilities. Robots can be used to efficiently carry out routine tasks without making the mistakes that a human would because of fatigue. Also robots can be used in environments which are harmful to the health of humans.

Mechanically most of the present day robots have arms consisting of rigid members connected by hinged or pivoted joints. To move such an arm precisely, the movements of its various members have to be coordinated. Such robots are slow-acting because of the inertia of the members and the time required to compute and control position.

A different approach using a light-weight, highly flexible, fast moving arm is made in this research. Such an arm is analogous to an elephant's trunk which has been analyzed recently [3]. It was observed that trained elephants can efficiently coil their trunks around heavy loads and lift them to desired positions. The present study simulates the action of an elephant's trunk within the limits of structural feasibility. The robotic arm is a continuous manipulator of rubber or polymeric material, consisting of hollow cylindrical elements joined end to end. The elements are selectively reinforced so that they bend, twist or extend when pressurized. Theoretical derivations and computer programs are written to evaluate the internal pressure required in each of the elements such that the arm can coil around a load and lift it to a desired position.

This research also deals with another important aspect of robotics. As machines cannot reason or think like humans, the robot has to be programmed to anticipate the various options or remedial measures, in advance of motion. Thus some aspects of Artificial Intelligence are incorporated into this research. A new Learning Program evaluates the end moments to be applied to 
each of the elements in order to lift and position the end load. This program's database is updated appropriately each time the program is run, thereby increasing the apparent knowledge and efficiency of the program in performing load positioning calculations.

The theoretical derivations for the mechanical behavior of the pressurized elements, the programming logic, and some numerical results for a typical, flexible robotic arm are discussed.

ANALYSIS OF A TYPICAL ELEMENT

The yeometry of a typical element is shown in Figure 1. It is a thin walled cylinder with the ends sealed and with reinforcement on the underside. The element is modelled as a cantilever beam of a nonlinear material. As in [4], finite deflections are considered. Given the applied end moment due to internal pressure, the horizontal and vertical deflections of the end of the beam are evaluated. The radius of curvature of the deflected beam is also computed. In this analysis, [5] was also used.

The hollow, cylindrical section is made out of a synthetic, nonlinear material for which the stress strain relationship is as follows

$$
\sigma=A \varepsilon+B \varepsilon l / n
$$

where $A, B$ and $n$ are material constants.

The synthetic material can withstand high strains (up to 200\%) without failure. The reinforcement is made of high strength steel shim or synthetic fibers with high tensile strength (Kevlar, for instance) as shown in Figure 2. The reinforcement provides the strength to the structure. The neutral axis for the composite element passes through the reinforcement because its stiffness is much greater in maynitude than that of the synthetic cylindrical section. For this reason with the application of internal pressure and thus an end moment, the element bends as shown in Figure 1. The reinforcement is assumed to be a linear material. 


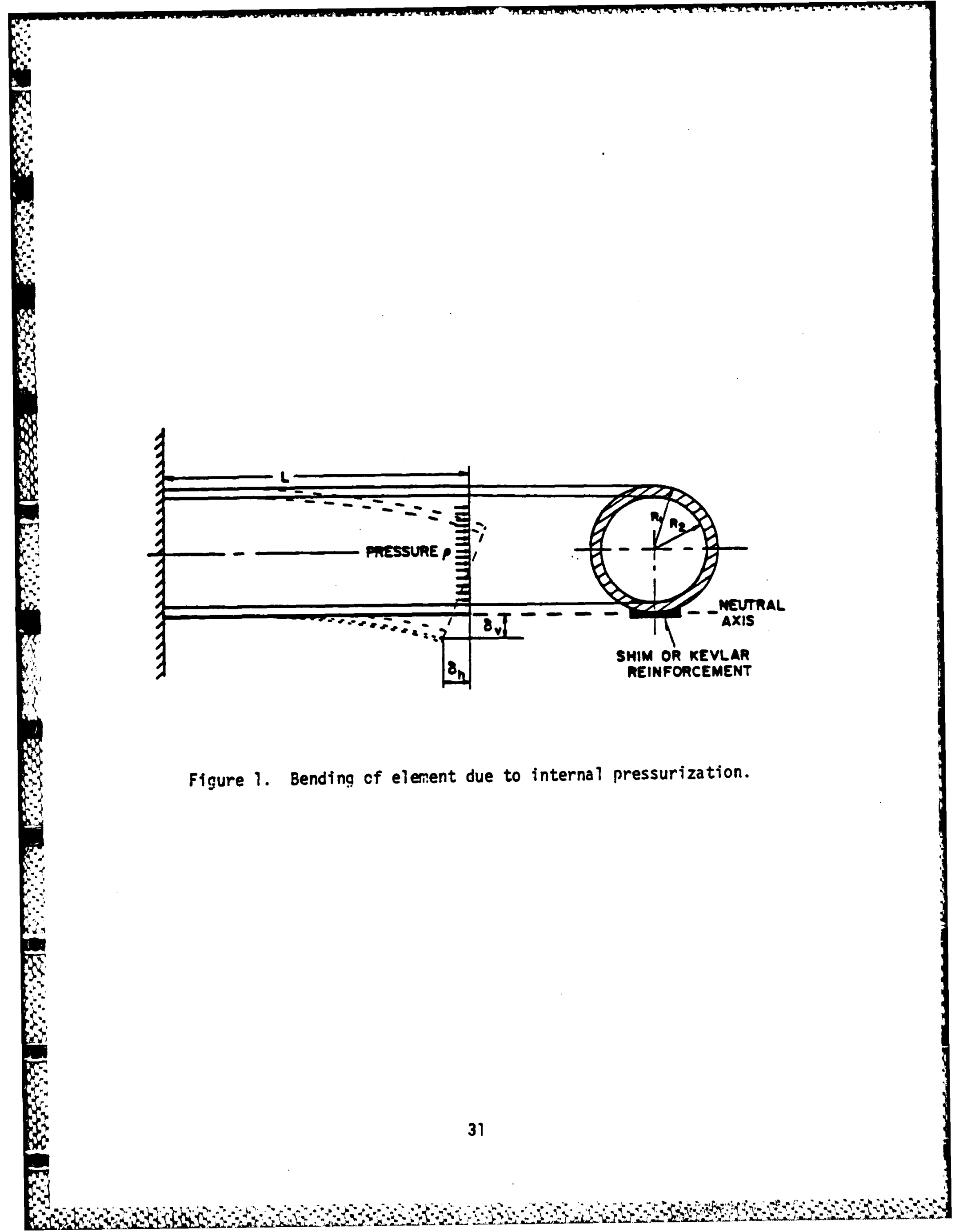



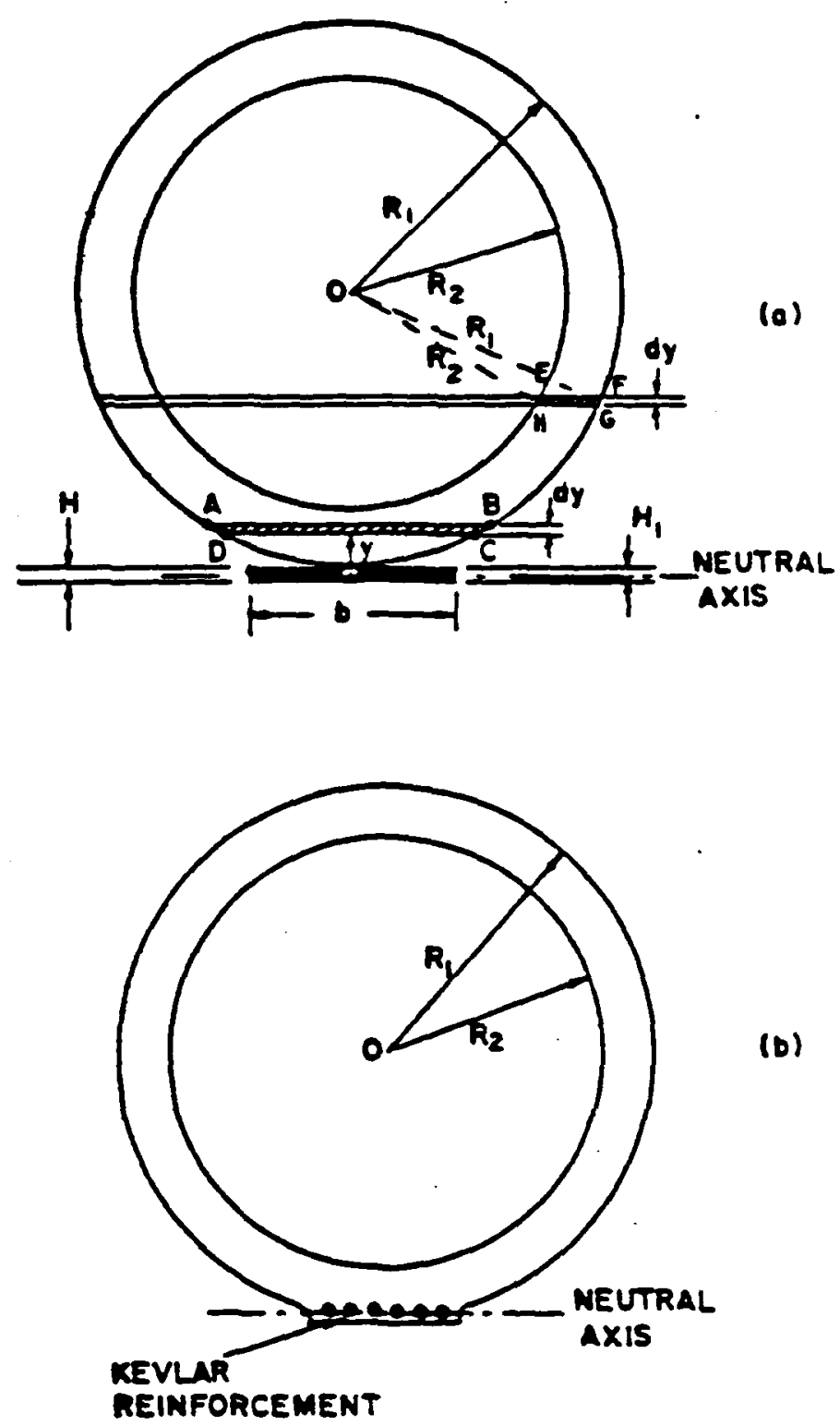

(b)

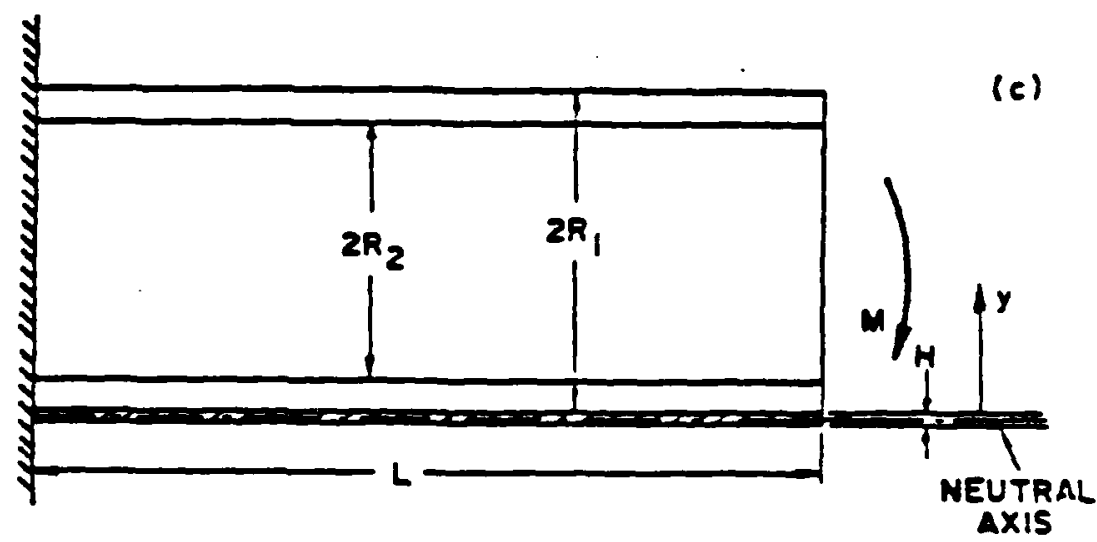

Figure 2. Cross-sectional vieus of element with shim (a); and with Kevlar reinforcement (b); side viel:! (c). 


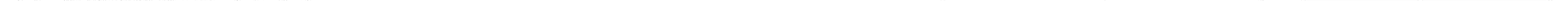




$$
\frac{1}{\rho}=\frac{M_{C}-\xi}{\alpha+\beta}
$$

where

$$
\begin{gathered}
\xi=\left(\frac{1}{\rho}\right)^{1 / n} n \\
\frac{1}{\rho}=\frac{y^{\prime \prime}(x)}{\left\{1+\left[y^{\prime}(x)\right]^{2}\right\}^{3 / 2}}
\end{gathered}
$$

Equating Equations (1) and (2) we get

$$
\frac{y^{\prime \prime}(x)}{\left\{1+\left[y^{\prime}(x)\right]^{2}\right\}^{3 / 2}}=\frac{M_{C}-\xi}{\alpha+\beta}
$$

Equation (3) is a second order differential equation which can be integrated directly with respect to $x$ to yield

$$
\frac{y^{\prime}(x)}{\left\{1+\left[y^{\prime}(x)\right]^{2}\right\}^{1 / 2}}=\frac{\left(M_{c}-\xi\right)}{(\alpha+\beta)} x+c_{1}
$$

The constant of integration, $c_{1}$, can be evaluated using the fact that $y^{\prime}(0)=$ 0. Thus $c_{1}=0$ and

$$
\frac{y^{\prime}(x)}{\left\{1+\left[y^{\prime}(x)\right]^{2}\right\}^{1 / 2}}=\frac{\left(M_{c}-\xi\right)}{(\alpha+\beta)} x
$$

Solving for $y^{\prime}(x)$ yields

$$
y^{\prime}(x)=\frac{\left(M_{c}-\xi\right) x}{\sqrt{(\alpha+B)^{2}-\left(M_{c}-\xi\right)^{2} x^{2}}}
$$

By integrating once more one obtains

$$
y(x)=\frac{(\alpha+\beta)-\left[(\alpha+\beta)^{2}-\left(M_{c}-\xi\right)^{2} x^{2}\right]^{1 / 2}}{\left(M_{c}-\xi\right)}
$$

where the constant of integration has been evaluated using the condition that $y(0)=0$ 
To evaluate the horizontal deflection, $\delta_{h}$, at the free end of the beam, as shown in Figure 3, the equation for the arc length is used. The total length then is

$$
L=\int_{0}^{L-\delta_{h}} \sqrt{1+\left[y^{\prime}(x)\right]^{2}} d x
$$

From Equations (5) and (6) we obtain

$$
L=\int_{0}^{L-\delta_{h}} \frac{\alpha+\beta}{\sqrt{(\alpha+\beta)^{2}-\left(M_{C}-\xi\right)^{2} x^{2}}} d x
$$

This integral can be evaluated using the trignometric substitution.

$$
\begin{aligned}
x & =\frac{\alpha+\beta}{M_{c}-\xi} \sin \theta \\
d x & =\frac{\alpha+\beta}{M_{c}-\xi} \cos \theta d \theta
\end{aligned}
$$

From Equation (8)

$$
\theta=\sin ^{-1}\left[x \frac{\left(M_{C}-\xi\right)}{(\alpha+\beta)}\right]
$$

when

$$
\begin{gathered}
x=L-\delta_{h} \\
{ }_{\left(\text {at } x=L-\delta_{h}\right)}=\bar{\theta}=\sin ^{-1}\left[\left(L-\delta_{h}\right) \frac{\left(M_{c}-\xi\right)}{(\alpha+\beta)}\right]
\end{gathered}
$$

Using Equations (9) and (10) from Equation (7) we get

$$
\begin{aligned}
L & =\int_{0}^{\bar{\theta}} \frac{(\alpha+\beta)^{2} \cos \theta}{\left(M_{c}-\xi\right) \sqrt{(\alpha+\beta)^{2}-(\alpha+\beta)^{2} \sin ^{2} \theta}} d \theta \\
& =\frac{(\alpha+\beta)}{\left(M_{c}-\xi\right)} \overline{0}_{0}^{-} d \theta \\
& =\frac{(\alpha+\beta)}{\left(M_{c}-\xi\right)} \sin ^{-1}\left[\left(L-\delta_{h}\right) \frac{\left(M_{c}-\xi\right)}{(\alpha+\beta)}\right.
\end{aligned}
$$




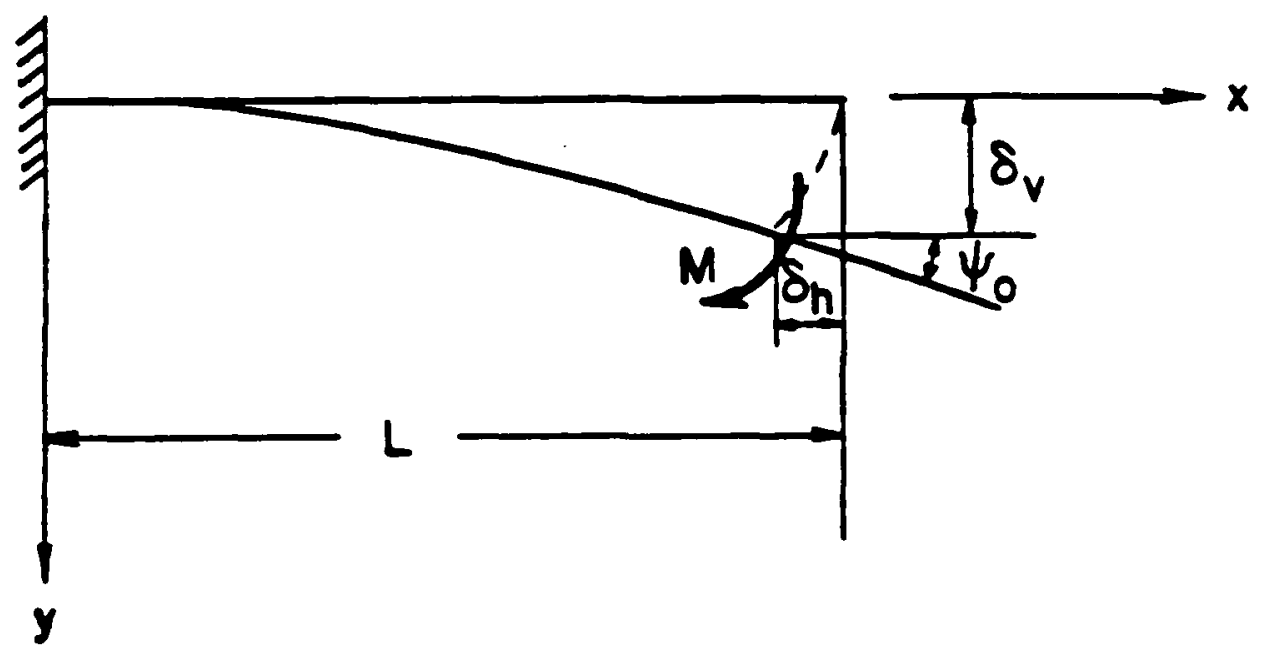

Figure 3. Cantilever beam subjected to an end moment. 


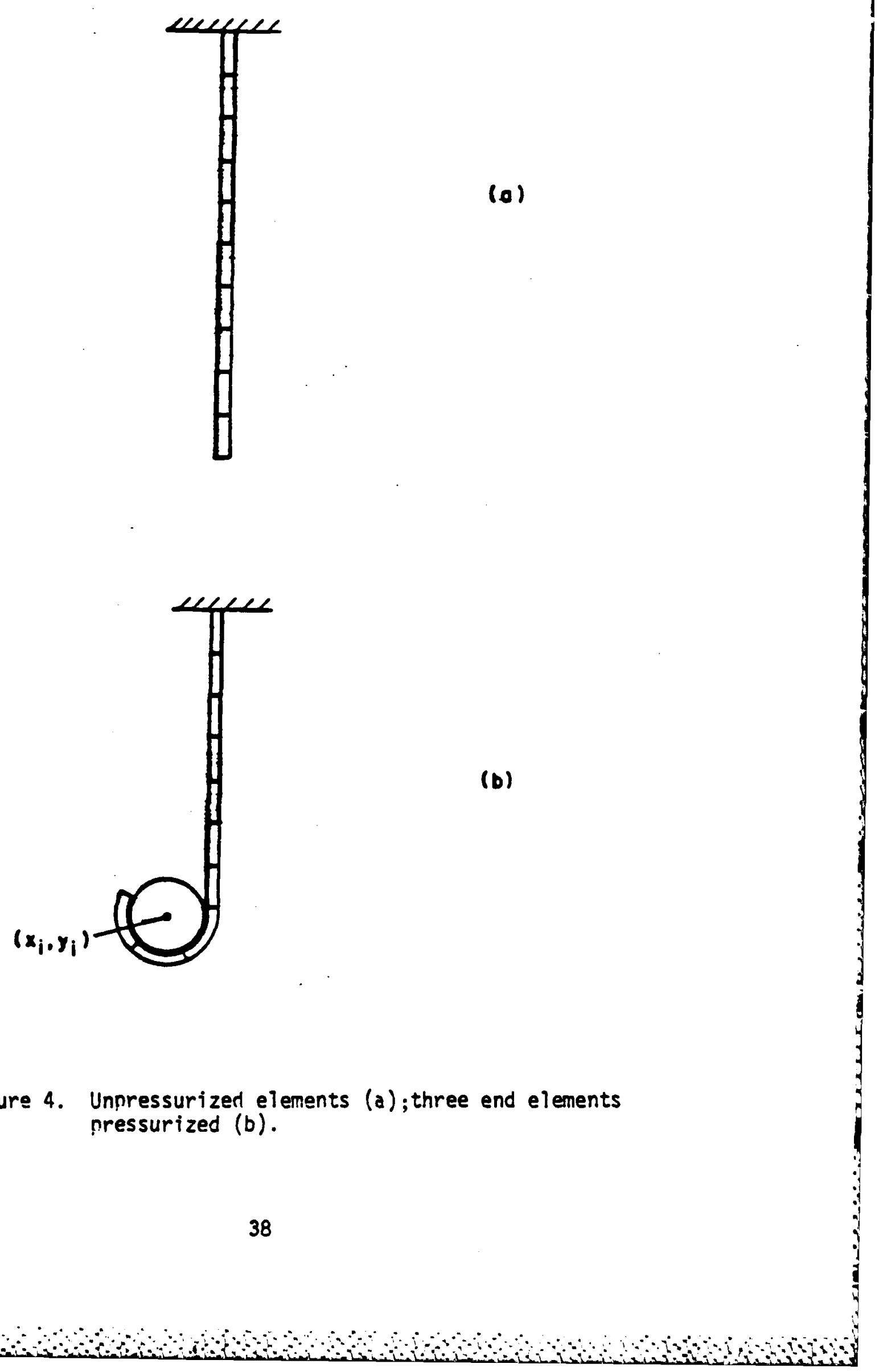




\section{Derivation to Transfer Local End Coordinates to Global End Coordinates}

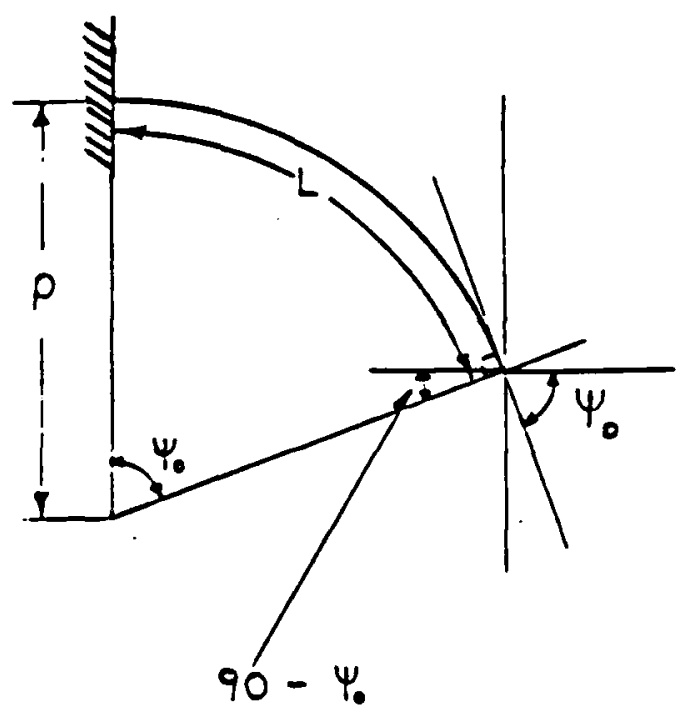

As the bending moment is constant for an element, the radius of curvature $p$ is constant. It follows from the sketch above that

$$
\Psi_{0}=\frac{L}{\rho}
$$

As shown in Figure 5 a for the first element the global and local axis coincide.

For the remaining elements the relationship between the local and glooal axis system is as follows.

$$
\left[\begin{array}{l}
x \\
y
\end{array}\right]=\left[\begin{array}{l}
x_{2} \\
y_{2}
\end{array}\right]+\left[\begin{array}{cc}
\cos x_{0} & \sin x_{0} \\
-\sin x_{0} & \cos x_{0}
\end{array}\right]\left[\begin{array}{l}
x_{1} \\
y_{1}
\end{array}\right]
$$

where 

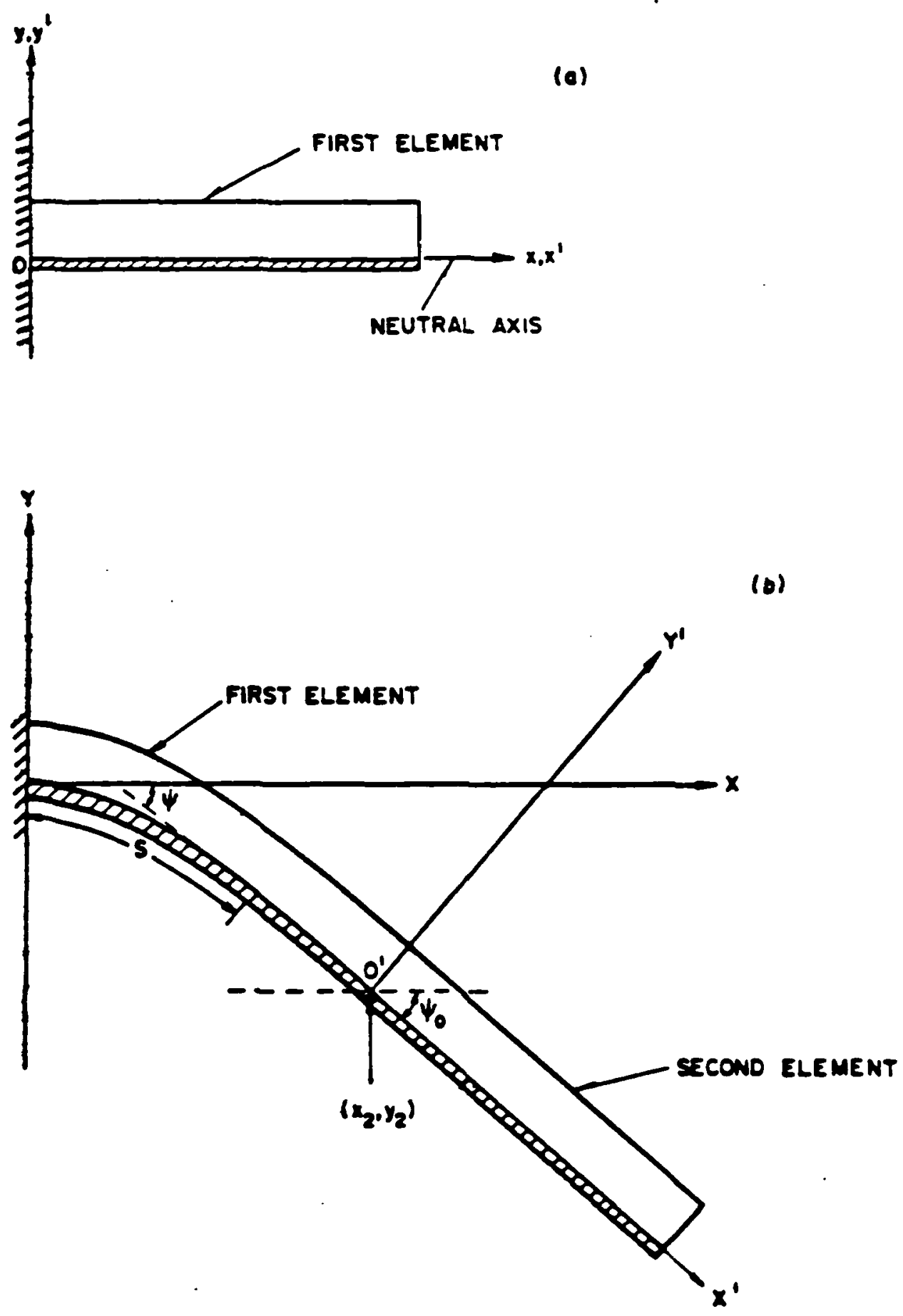

Figure 5. First element showing coincidence of local and global axes (a); and second element showing displaced local axis (b). 
$\psi_{0}$ is the anyle which the $x^{\prime}$ axis, of the local coordinate axis system of the element, makes with the $x$ axis of the global coordinate axis system.

$x_{2}, y_{2}$ are the coordinates of the oriyin of the local coordinate axis system in terms of the global coordinate axis system.

$x_{1}, y_{1}$ are the coordinates of any point $P$ in the local coordinate axis system.

$x, y$ are the coordinates of the same point $P$ in the global coordinate axis system.

In our case the point $P$ is the end point of the deflected element.

$$
\begin{aligned}
& x_{1}=L-\delta_{h} \\
& y_{1}=-\delta_{v}
\end{aligned}
$$

where

$$
\begin{aligned}
& \delta_{\mathrm{h}}=\text { horizontal deflection } \\
& \delta_{v}=\text { vertical deflection }
\end{aligned}
$$

For the next element

$$
y_{\text {on }}=\psi_{o c}+\frac{L_{c}}{\rho_{C}}
$$

The subscripts $n$ and $c$ mean next and current, respectively. The oriyin for the local coordinate axis system for the next element, is the end coordinates of the current element

$$
\begin{aligned}
& x_{2 n}=x_{c} \\
& y_{2 n}=y_{c}
\end{aligned}
$$

Now using the transformation matrix, the end coordinates of the next element can be converted from the local coordinate axis system to the global coordinate axis system. Similarly, this can be done for all the remaining elements. 
Evaluation of End Moments

It is required to compute the end moments to be applied on each of the elements such as to move the load from its initial position to the final desired position.

An example of a manipulator arm is shown in Figures $4 a$ and $4 b$. The first five elements have the shim (or reinforcement) on the right side whereas the remaining five elements have it on the left side. When these elements are pressurized there will be a reversal of curvature after the fifth element. Also by positioning the elements in this order, the arm has added capability in moving the load over a wider region.

Initially the load is assumed to have no mass. Then the end moments $M_{\rho}$, to elastically bend the arm and move the load to its desired position are computed.

Secondly, after the arm has reached its final configuration, we know the coordinates of the end point of each element and also the coordinates of the center of gravity of the load. Thus the additional moment due to the load $M_{\ell}$, (the load is now supposed to have mass) can now be easily evaluated.

The total moment $\quad M_{t}=M_{p}+M_{l}$

Evaluation of $M_{\rho}$

It is observed that there is no unique set of $M_{\rho}$, which can shift the load from its initial to the final position. The moments in some elements could be increased whereas the moments in some other elements could be decreased and the load could possibly be still in the same position. To overcome this problem, an iterative procedure has to be used. The iteration steps are as follows. 


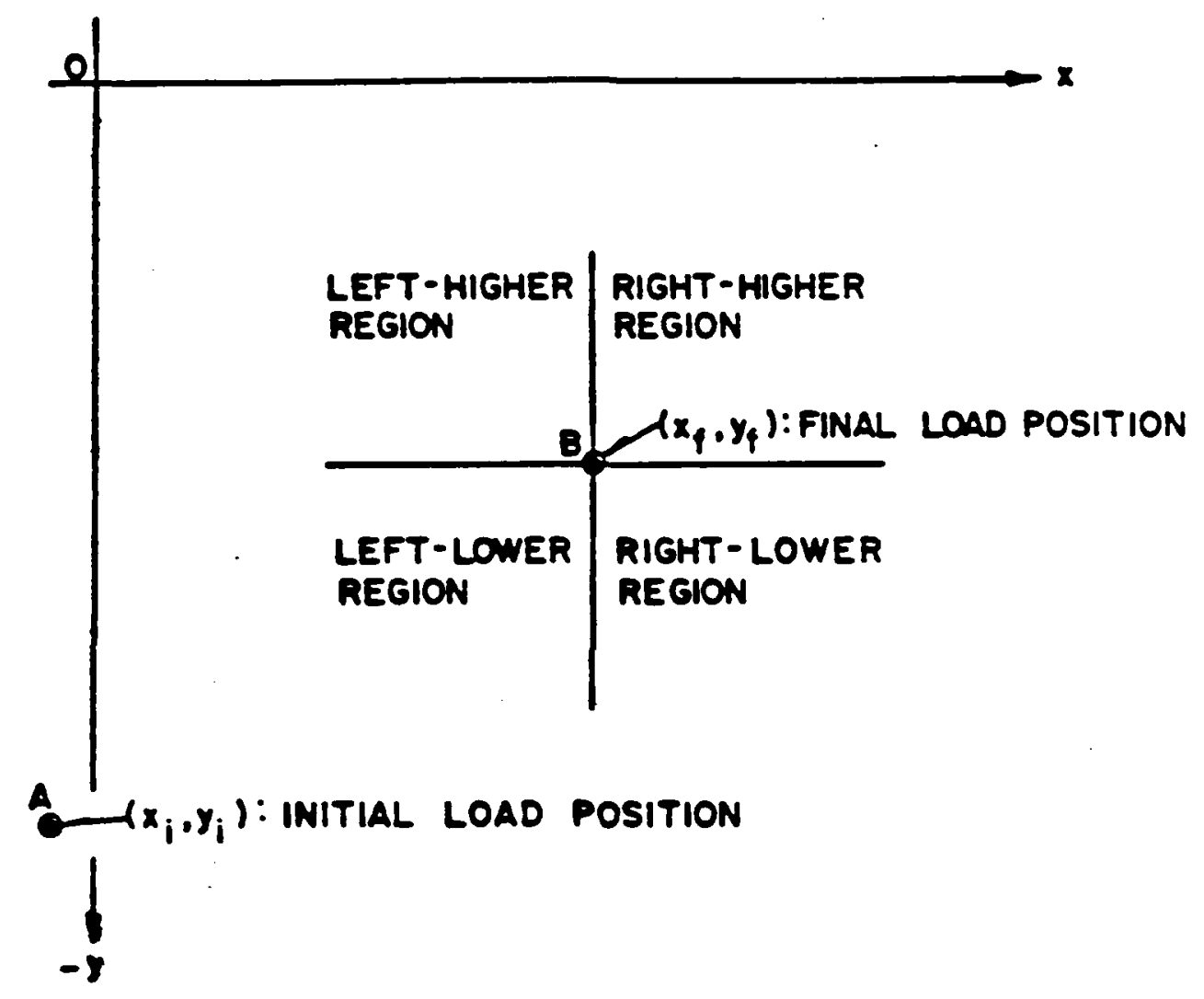

Figure 7. The four regions in which the load could be currently positioned. 


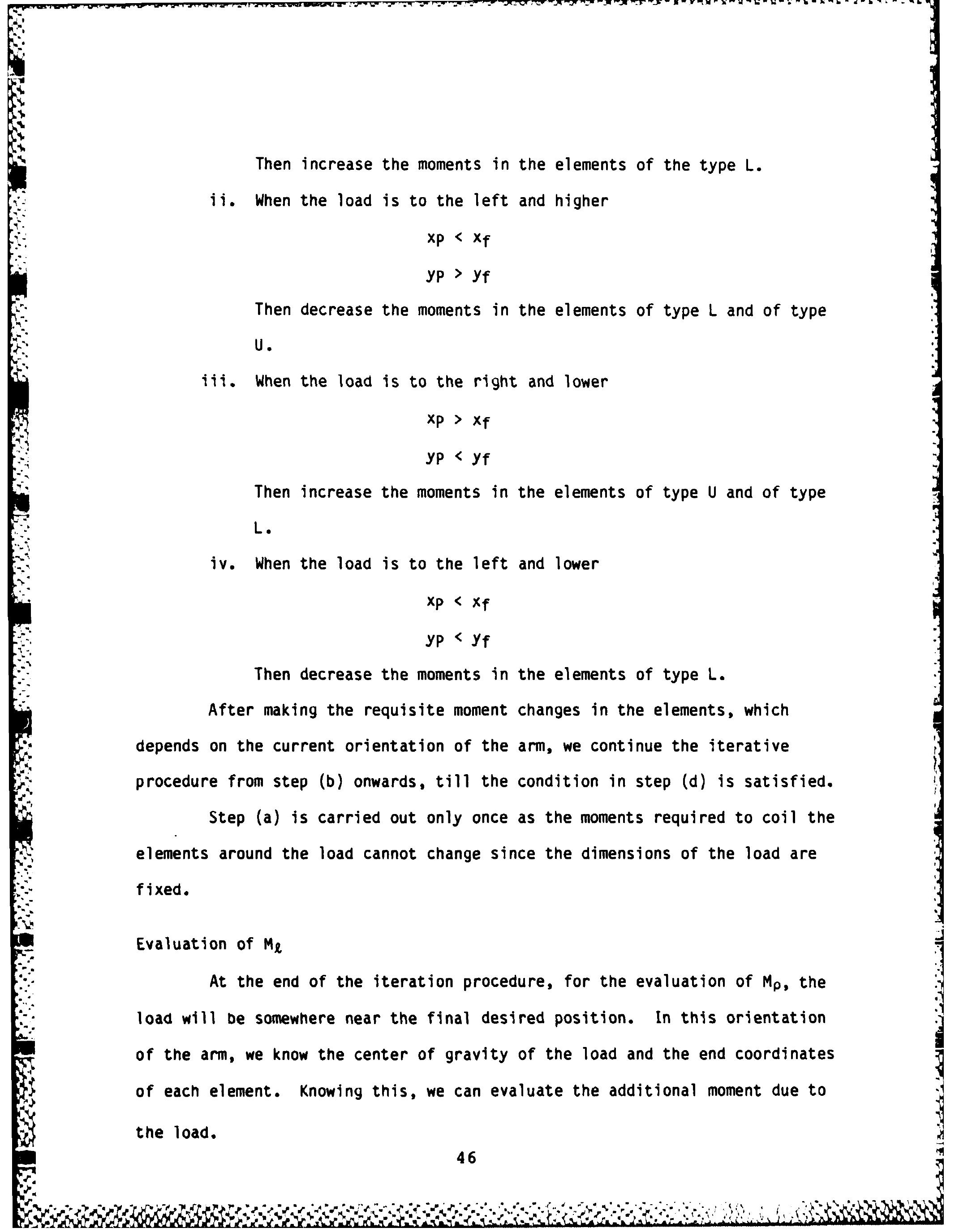


Since we do not precisely know the point of contact between the load and the last 3 elements coiled around it, we assume the worst case and apply an additional moment as given below.

$$
M_{\ell}=W_{1}\left(R_{1}+R_{\ell}+H\right)
$$

(For the last 3 elements $M_{l}$ is taken as same and is on the higher side)

We can compute the additional moments in the other elements from the free body diagram shown in Figure 8.

$$
M_{1}=W_{1}\left(R_{1}+R_{\ell}+H\right) \cos (\theta)
$$

$M_{\ell}$ for element type $U$, say element 5 is

$$
M_{\ell}=W_{1}\left(x_{c}-x_{d}\right)-M_{1}
$$

$M_{\ell}$ of element type $L$, say element 6 is

$$
M_{\ell}=M_{1}-W_{1}\left(x_{c}-x_{e}\right)
$$

Similarly we can evaluate $M_{\ell}$ for all the elements. Now for each of the elements we can evaluate

$$
M_{t}=M_{p}+M_{\ell}
$$

After the end moments required to be applied to each of the elements have been evaluated, the internal pressures required in each of the elements can easily be computed. As shown in Figure 9 (an arm with 3 elements only), there are three end moments, $M_{1}, M_{2}$ and $M_{3}$ and three different pressures $P_{1}, P_{2}$ and $P_{3}$

where

$$
\begin{aligned}
& M_{3}=P_{3} \pi R_{23}^{2} d_{3} \\
& M_{2}=\left(P_{2}-P_{3}\right) \pi R_{22}^{2} d_{2} \\
& M_{1}=\left(P_{1}-P_{2}\right) \stackrel{2}{2} R_{21}^{2} d_{1}
\end{aligned}
$$

where $R_{21}, R_{22}, R_{23}$ are the internal radii and $d_{1}, d_{2}, d_{3}$ are the distances between the center of pressure and the neutral axis for the three elements. 


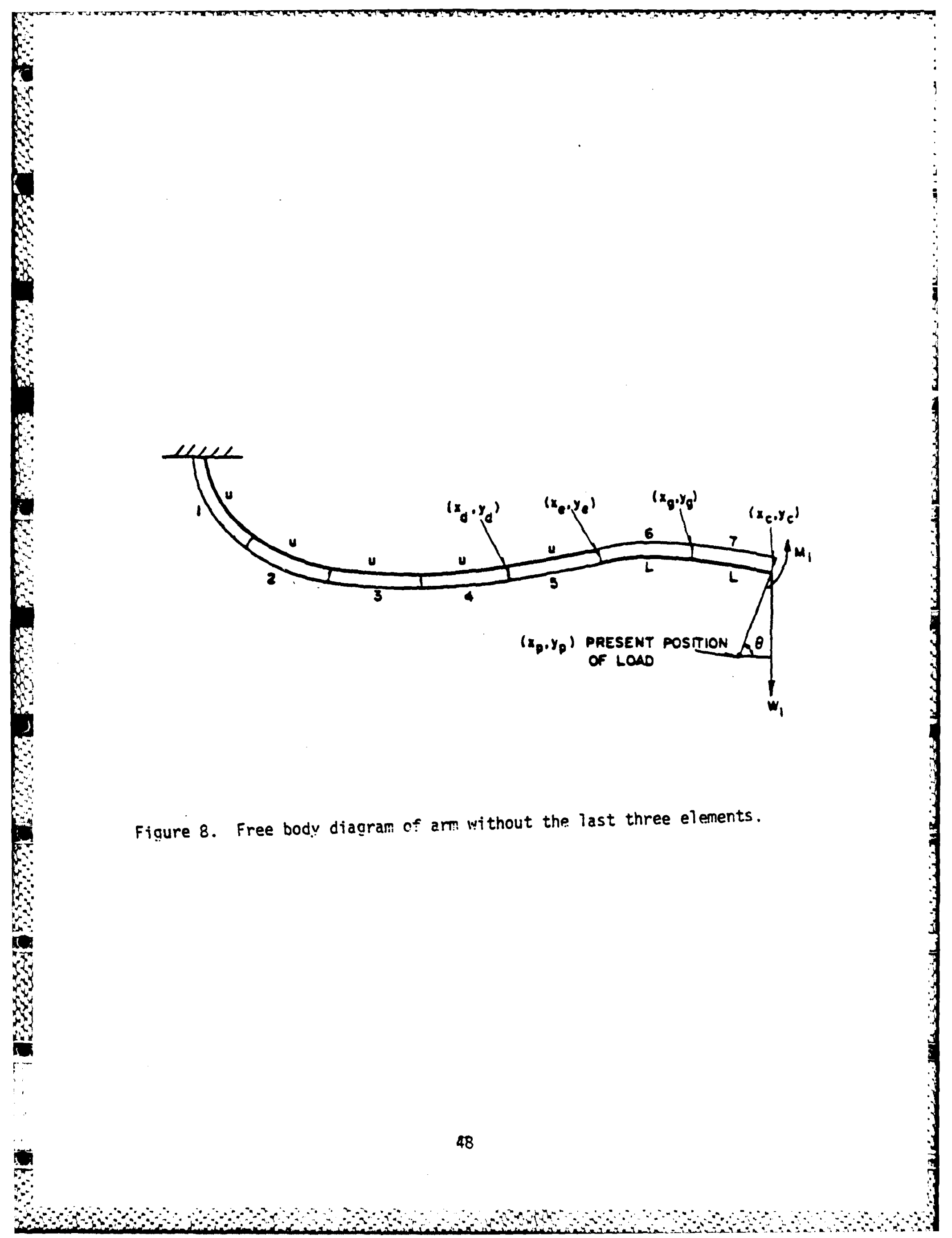




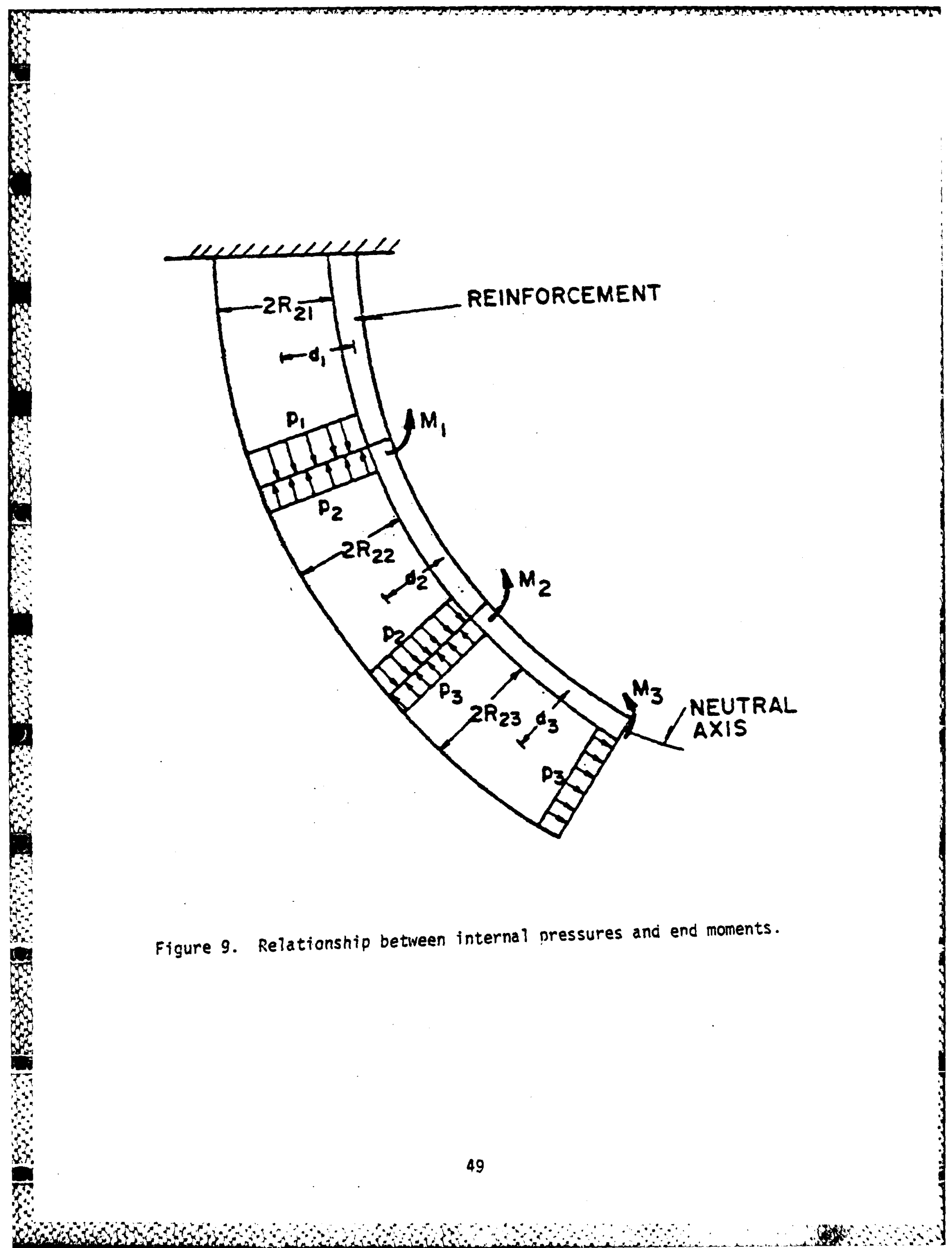




\section{IMPLEMENTATION OF THE LEARNING CONCEPT}

A FORTKAN proyram has been written to evaluate the end moments required in each of the elements, to move the load to its desired position. As mentioned in the previous chapter, apart from other input, initial guess end moment values $M_{\rho}$ are required to start up the iterative procedure.

It was observed that a faster converging solution was achieved (i.e. number of iterations were reduced), the closer the guess values were to the actual solution. Also the user who wants to use the robot, initially has little or no idea about what guess values to provide. Therefore the user is liable to input guess values which could lead to numerous iterations and thereby waste expensive computer time.

As a remedial measure, it was decided that the proyram instead of the user should decide about the guess values. Thus the program was modified into a 'Learning Program'. The program has a data structure, which for different initial to final load positions, has the corresponding required end moment values $M_{p}$. Now given an initial and final load position, the program searches through its data structure to locate the 'guess' end moment values to start the iterative procedure. Everytime the proyram is run, at the end of the iterations, the data structure is updated appropriately, thereby increasing the apparent knowledge of the program.

A FORTRAN program has been written, which carries out all the iterative computations, matrix transformations etc., discussed in this chapter. The program also takes care of another important aspect, i.e. the user does not have to provide the initial guess end moment values $M_{p}$, to start the iterative procedure. As discussed in the next chapter, the program searches through its database to come up with the requisite guess values. 
The data structure required to implement this 'Learning Concept', could have been appropriately implemented in a language like Pascal using tree structures. In our case as the remaining programs were in FORTRAN, we persisted with FORTRAN and had to use a five subscripted array as the data structure.

Data Structure and Search Logic:

As shown in Figure 10, given the coordinates of the initial and final positions of the load, we can uniquely define the initial and final position of the load, by the four parameters RAD, $\phi, \theta$ and DIS. The four parameters are defined as follows

$$
\begin{aligned}
\text { RAD } & \left.=\left[x_{j}\right)^{2}+\left(y_{i}\right)^{2}\right]^{1 / 2} \\
\phi & =\operatorname{Tan}^{-1}\left[\frac{x_{j}}{y_{j}}\right] \\
\text { DIS } & =\left[\left(x_{f}-x_{j}\right)^{2}+\left(y_{f}-y_{j}\right)^{2}\right]^{1 / 2} \\
\theta & =\cos ^{-1}\left[\frac{\left(x_{f}-x_{j}\right)}{D I S}\right]
\end{aligned}
$$

For each set of four such parameters we have corresponding end moment values $M_{p}$. Therefore when we search for the most accurate guess values, from the available data structure, a search through each of the four parameters is required. To simplify the search procedure, a five subscripted array $D(I, J, K, L, N)$, was used where the

First subscript corresponds to - ' $\phi$ ' values

Second subscript corresponds to - 'RAD' values

Third subscript corresponds to - ' $\theta$ ' values

Fourth subscript corresponds to - 'DIS' values

Fifth subscript corresponds to - end moment values $M_{p}$. 


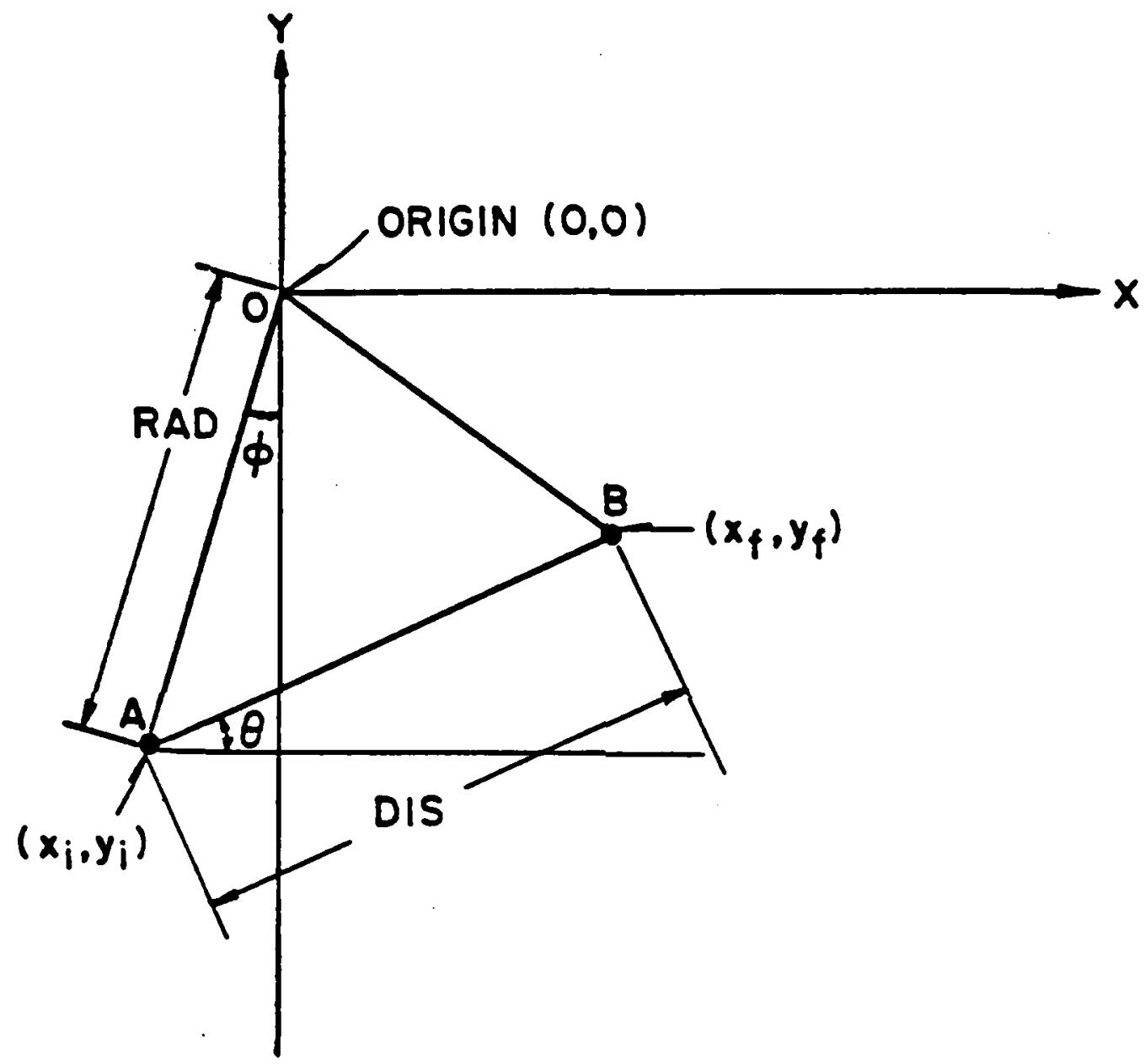

Figure 10. Initial and final position of load defined by $\phi, R A D, \theta$ and DIS with respect to the origin. 
Now it is shown in Figure 11 for each ' $\phi$ ' we have one or more corresponding 'RAD' values. Similarly for each 'RAD' we have one or more corresponding ${ }^{\prime} \theta^{\prime}$ values and for each ${ }^{\prime} \theta^{\prime}$ we have one or more corresponding 'DIS' values. Finally for each 'DIS' we have the corresponding end moment values for each element of the robotic arm.

Therefore given an initial load position $\left(x_{j}, y_{j}\right)$ and a final load position $\left(x_{f}, y_{f}\right)$, using Equations (20) to (23) we-can evaluate $\phi_{e}, R A D_{e}, \theta_{e}$ and $\mathrm{DIS}_{\mathrm{e}}$ (where subscript e signifies the evaluated parameters for which we wish to find best guess end moment values).

We can now search through the array $D(I, J, K, L, N)$. First, we compare ' $\phi$ ' with all the ' $\phi$ ' values in the array and choose the one which is closest to ' $\phi_{e}$ ' value. Let the chosen value be ' $\phi_{C}$ '.

Next we search through all the 'RAD' values, corresponding to ' $\phi_{C}$ ',

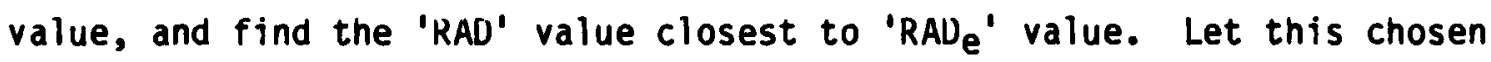
value be 'RADC'.

After this we search through all the ' $\theta$ ' values, corresponding to ' $R A D_{C}$ ' value, and find the ' $\theta$ ' value closest to ' $\theta e$ ' value. Let this chosen value be ' $\theta_{c}$ '.

Similarly we search through all the 'DIS', corresponding to ' $\theta_{c}$ ' value, and find the 'DIS' value closest to 'DIS $e^{\prime}$ value. Let this chosen value be 'DIS $c$ '.

The end moment values corresponding to 'OIS ${ }_{c}^{\prime}$, will be our initial guess moment values for the iterative procedure. This strategy of choosing the guess values is seen to work reasonably well.

At the end of the iterative procedure, the program computes the exact end moments required to move the load from position $\left(x_{i}, y_{j}\right)$ to position $\left(x f, y_{f}\right)$. 
The program now updates its data structure (Array $O(I, J, K, L, N)$ ) and $\phi_{e}, R A D_{e}, \theta_{e}$ and $D I S_{e}$ are inserted into the data structure in the proper places. As the array has all the values in increasing order of magnitude, insertion into the array is not difficult. The exact end moments computed by the program during the iterative procedure are also inserted into the array corresponding to $\phi_{e}, \mathrm{RAD}_{\mathrm{e}}, \theta_{\mathrm{e}}$ and $\mathrm{DIS} \mathrm{S}_{\mathrm{e}}$.

After the program has been run for several different, initial to final load positions, the data structure $D(I, J, K, L, N)$, after being repeatedly updated becomes sufficiently diverse. Now for a varied range of initial to final load positions, the guess values obtained will be sufficiently accurate.

The data structure is implemented by having a datafile. Everytime the program is run the values for array $D(I, J, K, L, N)$ are read in from this datafile. At the end of the program, after the array $D(I, J, K, L, N)$ has been updated, we overwrite the datafile with the present values of array $U(I, J, K, L, N)$. In this way the updated database is available for the next run of the program.

If the program has been run repeatedly which leads to the data structure becoming very large, the searching time may be too long. To avoid this, we can easily edit the datafile and remove the values in the range which are seldom used and leave only those values which are repeatedly used. Also after the datafile has reached an optimum size and we wish not to expand the datafile anymore, all that is required is to remove the 'write to datafile commands' from the program. 


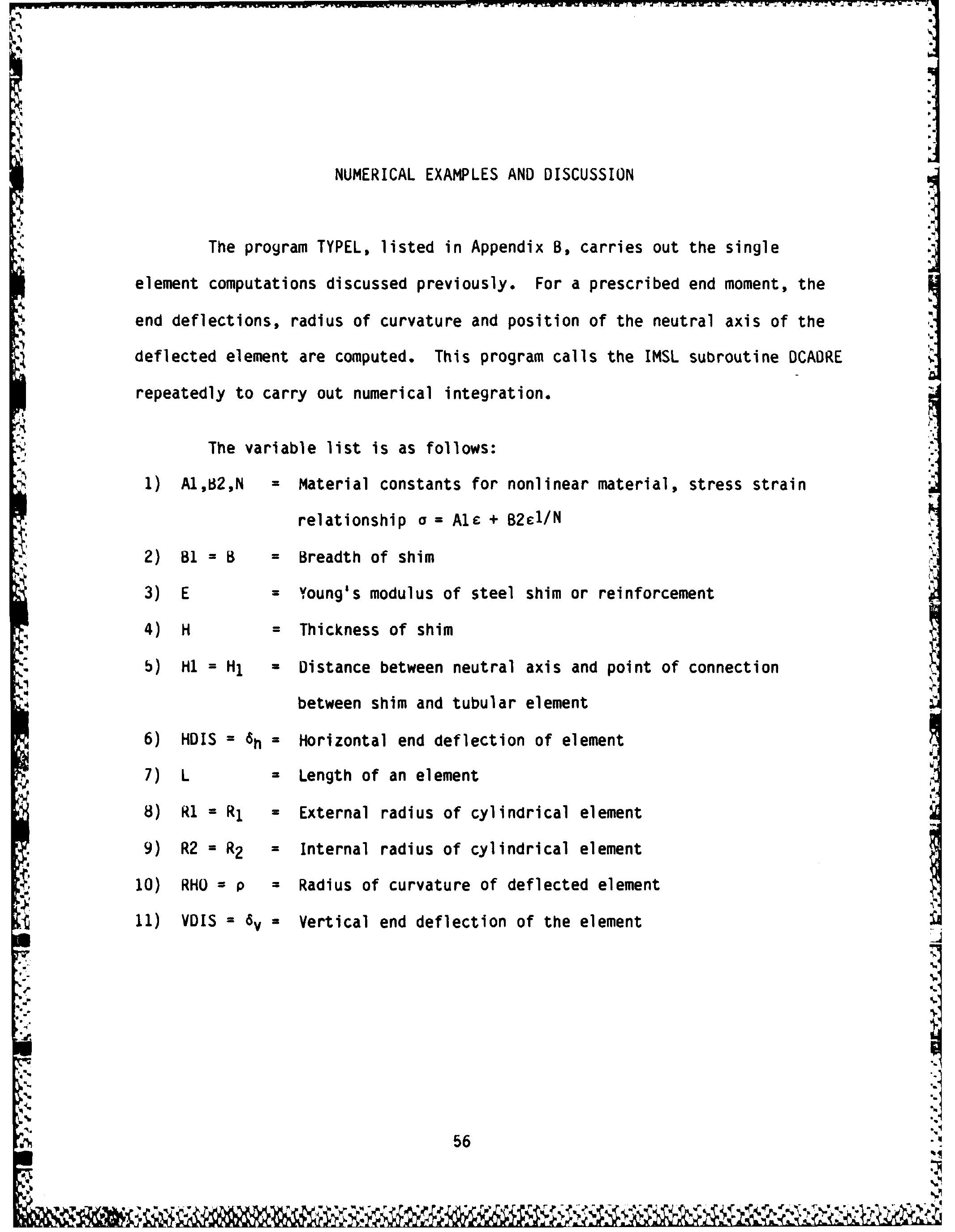


For an element such as shown in Figures 1 and 2, made of a polymeric material with a thin steel shim, the following numerical values were chosen as input parameters.

$\begin{array}{ll}N=0.2 & \text { (dimensionless) } \\ A 1=20.0 & \text { psi } \\ B 2=50.0 & \text { psi } \\ R 1=0.525 & \text { inches } \\ R 2=0.475 & \text { inches } \\ H=0.015 & \text { inches } \\ L=2.0 & \text { inches } \\ B 1=0.5 & \text { inches } \\ M=3.80 & \text { 1b in. } \\ E=3.0 \times 107 & \text { psi }\end{array}$

The results of the computations are as follows.

$\begin{array}{ll}H 1=0.00749 & \text { inches } \\ \text { RHO }=1.549 & \text { inches } \\ \text { VDIS }=1.121 & \text { inches } \\ \text { HDIS }=0.511 & \text { inches }\end{array}$

It is noted that the position of the neutral axis (H1) is nearly at the midheight of the steel shim. Further, the vertical deflection at the end of the element is about one-half the element length. In this case the internal pressure $p$ required to produce the chosen end moment $M$, in terms of the symbols of Figure 2, is

$$
p=\frac{M}{\pi R_{2}^{2}\left(R_{1}+H_{1}\right)}=\frac{3.80}{\pi(0.475)^{2}(C .525+0.00749)}=10.1 \text { psi }
$$

Note: The remaining variables in the program hold intermediate values and are of no significance to the user. 


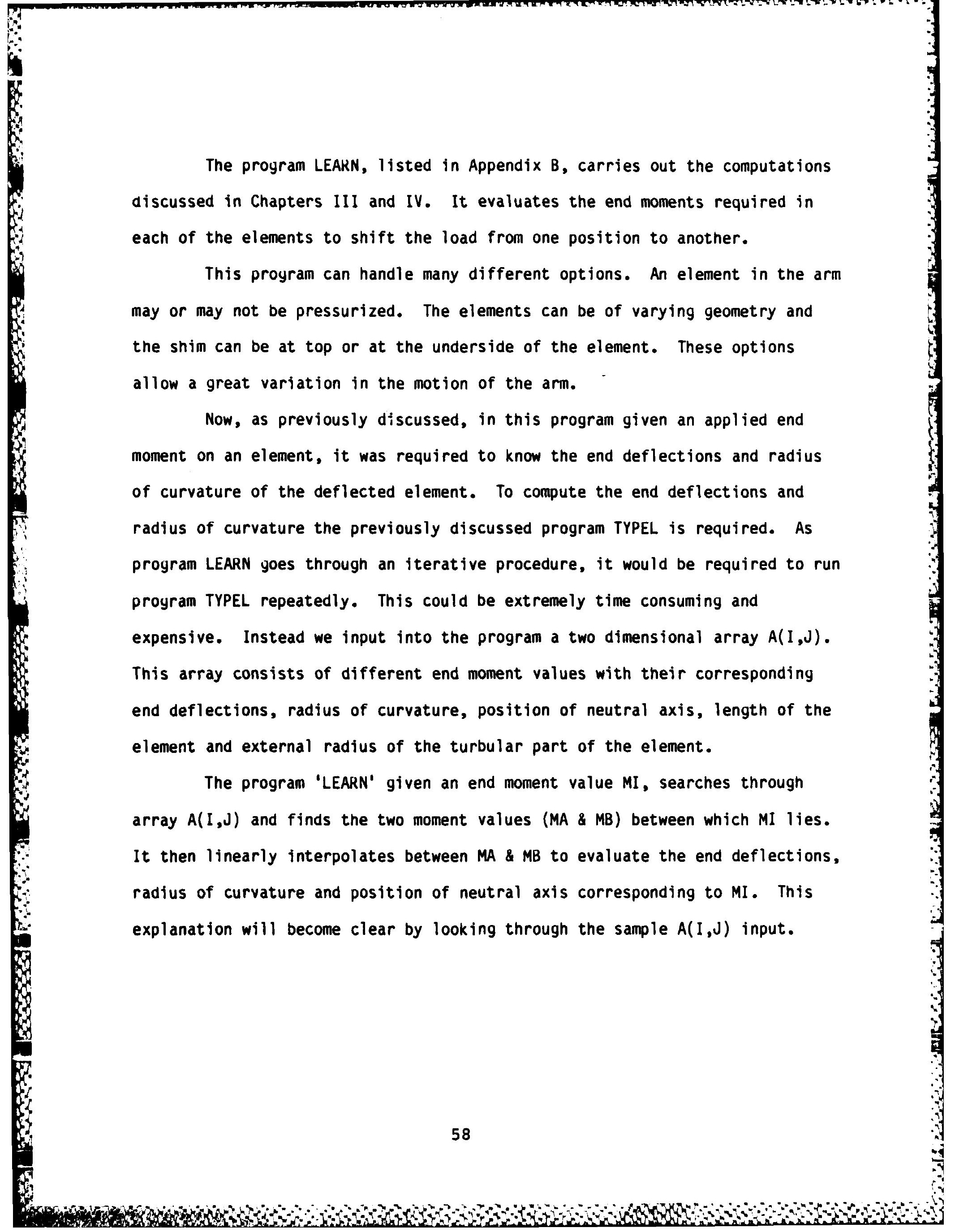


The variable list is as follows.

1) $A(I, J)$ = Array which has for different end moment values, the corresponding end deflections, radius of curvature, length of element, position of neutral axis and the external radius of element

2) ANG = End cumulative rotation up to that element in degrees

3) $D(I, J, K, L, M)=$ Holds the 'apparent knowledge' data structure

4) $\mathrm{H} \quad$ = Thickness of shim

5) $\mathrm{Hl} \quad=$ Distance between neutral axis and point of connection between shim and turbular part

6) HDIS = Horizontal end deflection of a particular element

7) IDIR = Contains the IMOT value of previous element

8) ImUT = $\quad-3$ means element deflects upwards

$=\quad+3$ means element deflects downwards

9) INCK = to fix position of global $x$ axis

10) IPI = +2 means element is pressurized

$=-2$ means element is not pressurized

11) IPCK = 0 means no pressurized elements in the arm up till now

$=1$ means pressurized element has been encountered

12) IPLTI = -1 means shim on right side

$=\quad+1$ means shim on left side

13) ITYPE = 1 means use array $A(I, J)$

$=2$ means use array $B(I, J)$

14) LN = Length of an element

15) $\mathrm{Nl}=$ Number of identical elements (geometrical and material properties identical and also same end moments) 
16) NN

17) NSHIF

18) NU

19) MM

20) R1

21) RHO

22) $R L$

23) SI

24) VDIS

25) W1

26) $X 2, Y 2$

27) $X 3, y 3$

28) $X B, Y B$

29) $X I, Y I$

30) XF, YF
$=$ number of elements in the arm

= shift in position of neutral axis between consecutive elements

$=$ number of elements with shim on top

$=$ number of data sets in $A(I, J), I=1$ to $M M$

= outer radius of cylindrical part

= radius of curvature of the neutral axis of an element

= radius of load

= cumulative end rotation

= vertical end deflection of a particular element

= weight of load

= end point, values for the current element w.r.t. neutral axis

= end point, values for the current element w.r.t. mid axis of element

$=$ coordinates of c.g. of load at end of each iteration

$=$ coordinates of $\mathrm{c.g}$. of load in its initial rest position

$=$ coordinates of c.g. of load in the final desired position

31) $X O R O(I), Y O R D(I)=$ coordinates of end points of the elements 


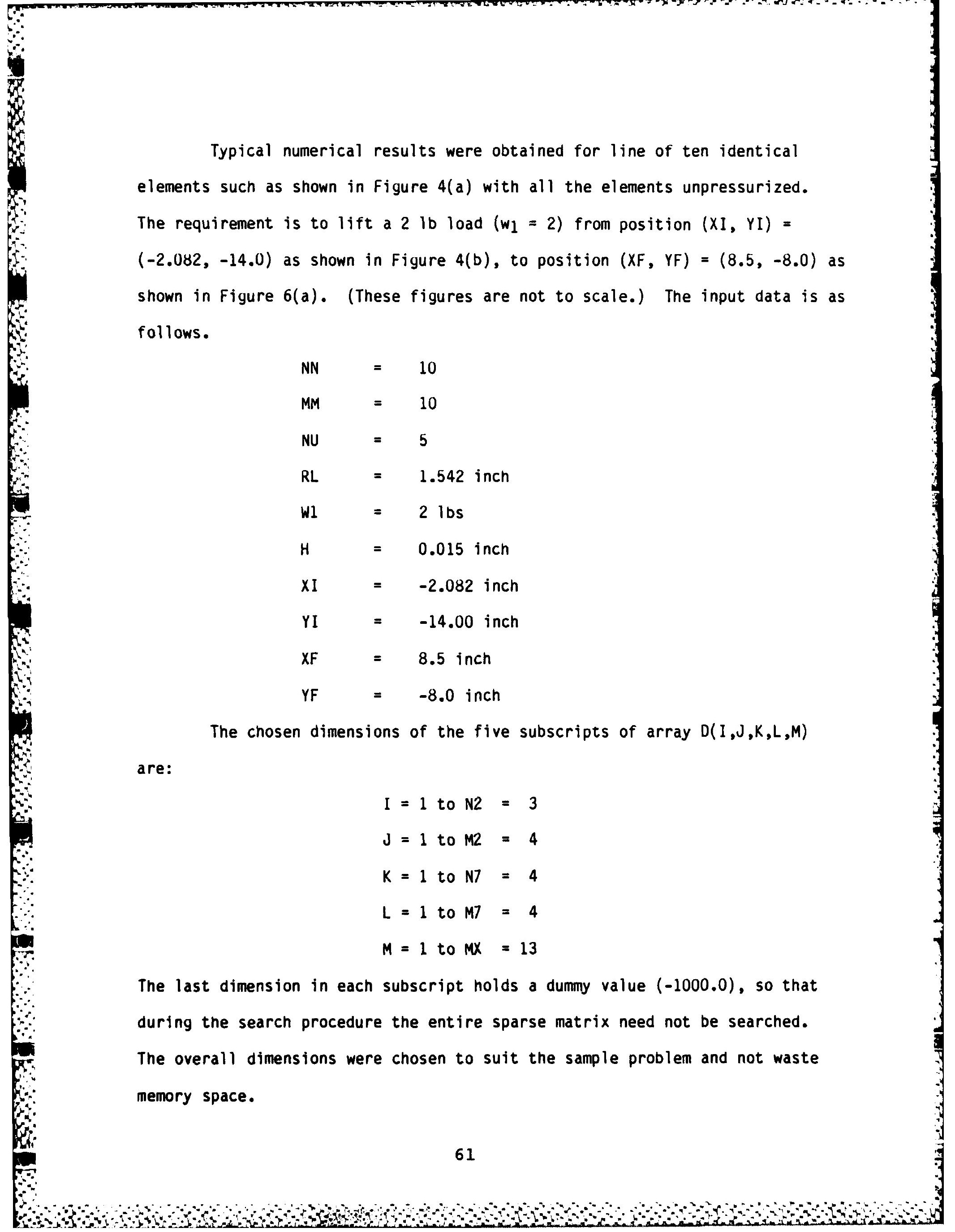


Specific data about each element has to be provided to the program. For example, IPLT1 specifies position of shim, IPI specifies whether the element is to be internally pressurized or not. The following table specifies the properties of all ten elements in this example problem.

$$
I=1 \text { to } N N \quad N N=10 \text { in this example }
$$

\begin{tabular}{ccccc}
\hline I TYPE (I) & IPI(I) & IPLTI(I) & N1(I) & IMOT1 (I) \\
\hline 1 & +2 & -1 & 1 & -3 \\
1 & +2 & -1 & 1 & -3 \\
1 & +2 & -1 & 1 & -3 \\
1 & +2 & -1 & 1 & -3 \\
1 & +2 & -1 & 1 & -3 \\
1 & +2 & +1 & 1 & +3 \\
1 & +2 & +1 & 1 & +3 \\
1 & +2 & +1 & 1 & +3 \\
1 & +2 & +1 & 1 & +3 \\
\hline
\end{tabular}


For elements having similar geometry and stiffness properties, we can repeatedly run program TYPEL to obtain the results tabulated below. Now during the iterative procedure in program LEARN, given the end moment value, we can by interpolation of the array $A(I, J)$ obtain the corresponding end deflections, radius of curvature and the other items in the table below.

Array $A(I, J)$

$$
\begin{aligned}
& I=1 \text { to } 10 \\
& J=1 \text { to } 7
\end{aligned}
$$

\begin{tabular}{|c|c|c|c|c|c|c|}
\hline $\begin{array}{l}\text { End } \\
\text { Moment } \\
(1 b-i n)\end{array}$ & $\begin{array}{c}\text { Radius of } \\
\text { Curvature } \\
\rho(i n)\end{array}$ & $\begin{array}{c}H 1 \\
(\text { in })\end{array}$ & $\begin{array}{l}\text { HDIS } \\
\text { (in) }\end{array}$ & $\begin{array}{l}\text { VDIS } \\
\text { (in) }\end{array}$ & $\begin{array}{l}\text { Length of } \\
\text { Element LN } \\
\text { (in) }\end{array}$ & $\begin{array}{c}\text { Radius of } \\
\text { Element Rl } \\
\text { (in) }\end{array}$ \\
\hline 1.02 & 5.3979 & $0.749255 \times 10^{-2}$ & 0.0455 & 0.3663 & 2.0 & 0.525 \\
\hline 1.265 & 4.3537 & $0.749259 \times 10^{-2}$ & 0.0696 & 0.4514 & 2.0 & 0.525 \\
\hline 1.705 & 3.2391 & $0.749247 \times 10^{-2}$ & 0.1247 & 0.5981 & 2.0 & 0.525 \\
\hline 1.915 & 2.8904 & $0.74924 \times 10^{-2}$ & 0.1558 & 0.6648 & 2.0 & 0.525 \\
\hline 2.2799 & 2.4409 & $0.749228 \times 10^{-2}$ & 0.2164 & 0.7745 & 2.0 & 0.525 \\
\hline 2.4085 & 2.3165 & $0.749221 \times 10^{-2}$ & 0.2394 & 0.8111 & 2.0 & 0.525 \\
\hline 2.634 & 2.130 & $0.7492 \times 10^{-2}$ & 0.2812 & 0.8702 & 2.0 & 0.525 \\
\hline 3.159 & 1.807 & $0.74916 \times 10^{-2}$ & 0.3839 & 0.9982 & 2.0 & 0.525 \\
\hline 3.798 & 1.549 & $0.7491 \times 10^{-2}$ & 0.5109 & 1.121 & 2.0 & 0.525 \\
\hline 4.587 & 1.346 & $0.7489 \times 10^{-2}$ & 0.6592 & 1.232 & 2.0 & 0.525 \\
\hline
\end{tabular}


The numerical results are summarized as follows.

\begin{tabular}{ccc}
\hline Element No. & $\begin{array}{c}\text { Total End Moment } \\
\text { to be Applied (lb in) }\end{array}$ & $\begin{array}{c}\text { Element } \\
\text { Pressure (psi) }\end{array}$ \\
\hline 1 & $0.181 \times 10^{2}$ & 262.33 \\
2 & $0.173 \times 10^{2}$ & 214.38 \\
3 & $0.149 \times 10^{2}$ & 168.54 \\
4 & $0.114 \times 10^{2}$ & 129.06 \\
5 & $0.721 \times 10^{1}$ & 98.86 \\
6 & $0.133 \times 10^{2}$ & 79.76 \\
7 & $0.502 \times 10^{1}$ & 76.24 \\
8 & $0.792 \times 10^{1}$ & 62.94 \\
9 & $0.792 \times 10^{1}$ & 41.96 \\
10 & $0.792 \times 10^{1}$ & 20.98 \\
\hline
\end{tabular}

It is to be noted that elements 8,9 and 10 have identical end moments. As the exact load distribution is not known, these elements are over-pressurized so that they coil around and grip the load more tightly than is required for lifting. The pressure $P_{j}$ in the $i$-th element whose end moment is $M_{j}$ was calculated from the following recursion relationships.

$$
M_{j}=\left(p_{j}-p_{j+1}\right) \pi R_{2}^{2}\left(R_{1}+H_{1}\right)
$$

It is observed that the pressures in the respective elements are higher for the ones closer to the support.

The elements in the sample problem were identical but if the arm consists of elements such that the cross-sectional area of elements decreases successively as they move away from the support (element 1 is attached to the support) then there would be no drastic decrease in pressure between successive elements. 
There are several possible extensions of this research. For instance, the proyramminy efficiency can be considerably improved if the program is rewritten in Pascal which offers the facility of tree type of data structure and dynamic storaye. By implementing a tree type of datastructure searching and insertion of data is yreatly simplified. Also, the mathematical model for plane motion as discussed here can be generalized to include out-of-plane manipulations. This can be done by the addition of torsion elements [6] at the base of element one. One torsion element can move the arm $\pm 180^{\circ}$ out of the plane whereas another torsion element can move the arm $+90^{\circ}$ in the plane. By judiciously pressurizing these torsion elements, the robotic arm can have a work space consisting of volume of revolutions in each of the four quadrants. 


\section{REFERENCES}

1. Critchlow, Arthur J., "Introduction to Robotics," Macmillan Publishing Company, New York, 1985.

2. Safford, E. L., "Handbook of Advanced Robotics," Tab Books Inc., 1982.

3. Wilson, J. F., Mahajan U., Croner, L. J., and Wainwright, S. A., "Mechanics of an Elephant Trunk: Theory and Measurements," (submitted for publication).

4. Lewis, G. and Monasa, F., "Large Deflections of Cantilever Beams of Non-Linear Materials of the Ludwick Type Subjected to an End Moment," International Journal of Non-Linear Mechanics, Vol. 17, No. 1, pp. 1-6, 1982.

5. Popov, E. P., "Mechanics of Materials," Prentice Hall International, Inc., Englewood $\mathrm{Cl}$ iffs, 1978.

6. Wilson, J. F. and Orgill, G., "Linear Analysis of Uniformly Stressed Orthotropic Cylindrical Shelis," (submitted for publication). 


\section{LINEAR ANALYSIS OF UNIFORMLY STRESSED, ORTHOTROPIC CYLINDRICAL SHELLS}

\section{ABSTRACT}

Within the framework of classical elasticity, the nonbuckled deformations are calculated for orthotropic, right circular, thin-walled cylinders under uniform load conditions. The principal direction of orthotropy follows parallel constant angle helices. Nondimensional system parameters involving four material constants and three loading conditions (internal pressure, longitudinal load and pure torque) are identified. Through parametric studies deformation patterns are calculated that are unique to orthotropy. Numerical examples illustrate that the proper selection of cylinder orthotropy can lead to designs with optimal deformations or load-carrying capacity. Results may be used for the design of robotic actuators driven by internal pressure. 


\section{INTRODUCTION}

Improvement in the mechanical performance of cylindrical or nearly cylindrical shell-type structures may be achieved by adding reinforcement or by making small modifications in the basic structural geometry. For instance, the high pressure capacity of fire hoses is due to the reinforcing effect of the helical fibers in the rubber walls. In other cases, the buckling resistance to longitudinal loading of thin-walled cylindrical columns can be greatly improved by the use of a fluted design, or a periodic variation of the radius around the circumference. On the other hand, there are cases where high deformations are desirable as, for instance, in cylindrical bellows used as pipeline expansion joints. Here, length changes of up to 100 percent may be achieved by the use of axisymmetric, periodic corrugations along the length of a basic cylindrical shape [1]. Thus, the most flexible directions of the bellows and the thin-walled fluted column are orthogonal.

The purpose of this paper is to present a unified continuum model of such thin-walled, cylindrical shells using directional material properties. Such continuum models are especially efficient for calculating overall, nonbuckling, elastic deformations for fiber-reinforced cylinders [2] and for uniformly loaded, anisotropic, cylindrical shells $[3,4]$. In the present study, classical elasticity is employed to predict the nonbuckled deformations for uniform, orthotropic right circular cylinders with thin walls, subjected to three types of uniform loads: a torque $T$ effecting rotation about the longitudinal axis, a longitudinal load $P$, and an internal pressure $P$. Strains along the geometric axes of symmetry are presented as functions of nondimensional system parameters involving these loads (applied separately and in combination), the cylinder geometry, four material properties, and the constant helix angle $\theta_{0}$ that defines the direction of maximum stiffness. See Figure 1. 

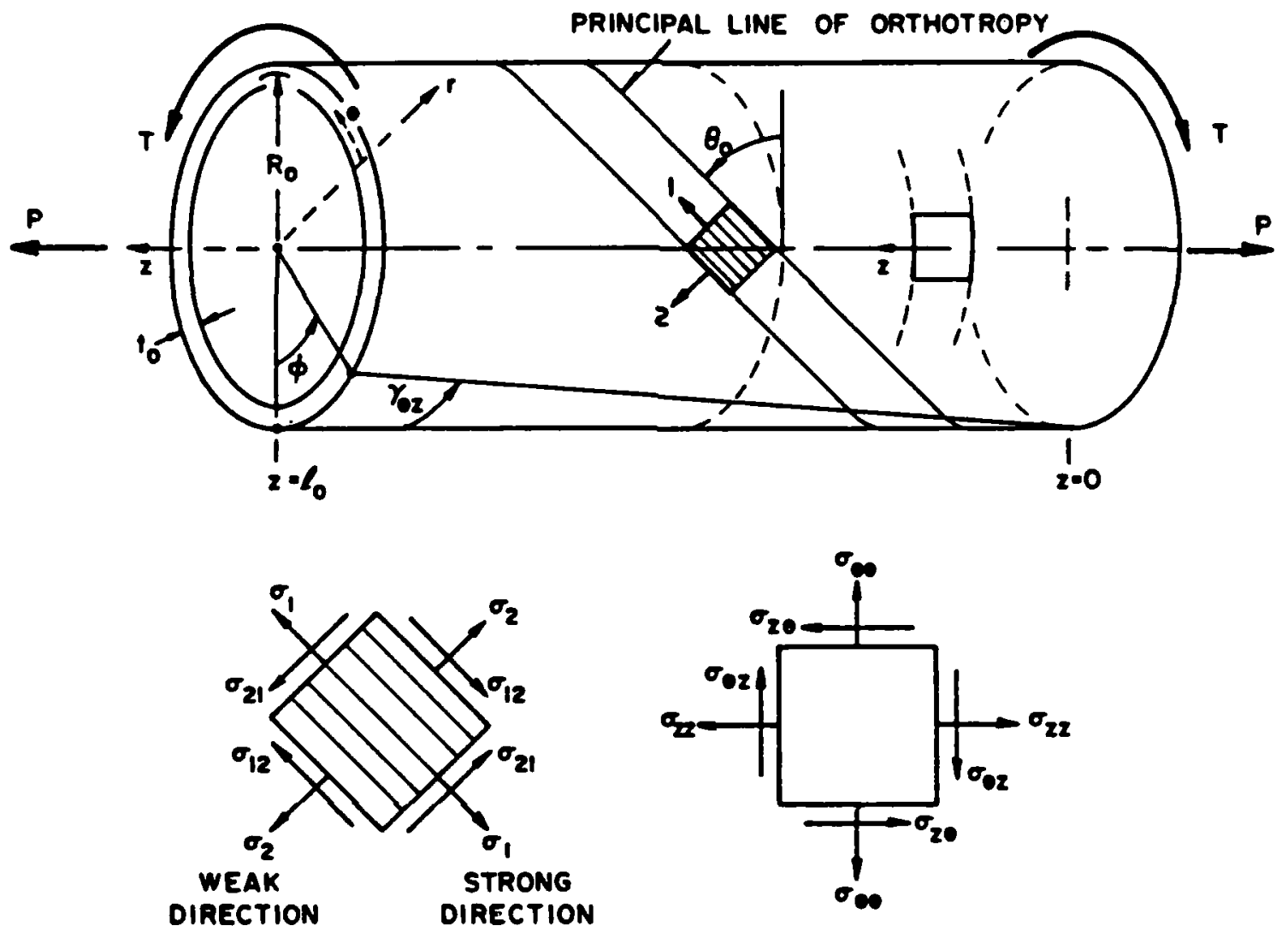

Figure 1 Definition of the orthotropic cylinder

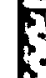


This continuum model may describe the overall deformation characteristics of fiber-reinforced tubes as well as tubes with corrugations at arbitrary helix angles, as long as the cylindrical shape is maintained under load. Results may be used in future designs of robotic actuators that twist $\left(40^{\circ}<\theta_{0}<80^{\circ}\right)$ or that only lengthen as for bellows $\left(\theta_{0}=0\right)$ when subjected to internal pressure.

\section{CONSTITUTIVE RELATIONSHIPS}

Following the development in [5], the constitutive law for an orthotropic, elastic solid that relates the six strain components to the six stress components is given by

$$
\left[\begin{array}{l}
\varepsilon_{11} \\
\varepsilon_{22} \\
\varepsilon_{33} \\
r_{12} \\
r_{13} \\
r_{23}
\end{array}\right]=\left[\begin{array}{cccccc}
\frac{1}{E_{11}} & -\frac{v_{21}}{E_{22}}-\frac{v_{31}}{E_{23}} & 0 & 0 & 0 \\
-\frac{v_{12}}{E_{11}} & \frac{1}{E_{22}}-\frac{v_{32}}{E_{33}} & 0 & 0 & 0 \\
-\frac{v_{13}}{E_{11}} & -\frac{v_{23}}{E_{22}} & \frac{1}{E_{33}} & 0 & 0 & 0 \\
0 & 0 & 0 & \frac{1}{G_{12}} & 0 & 0 \\
0 & 0 & 0 & 0 & \frac{1}{G_{13}} & 0 \\
0 & 0 & 0 & 0 & 0 & \frac{1}{G_{23}}
\end{array}\right]\left[\begin{array}{l}
\sigma_{11} \\
\sigma_{22} \\
\sigma_{33} \\
\sigma_{23}
\end{array}\right]\left[\begin{array}{l}
\sigma_{12} \\
\sigma_{13}
\end{array}\right]
$$

Equation (1) is written with respect to the principal axis $(1,2,3)$. The $6 \times 6$ material matrix, whose elements are designated as $a_{i j}$, is determined by experiments. The strains and stresses of Equation (1) are redefined using single subscription, or 


$$
\begin{aligned}
& \varepsilon_{11}=\varepsilon_{1}, \\
& \gamma_{12}=\varepsilon_{4}, \\
& \sigma_{11}=\varepsilon_{2},
\end{aligned}
$$

Using this notation, Equations (1) become

$$
\varepsilon_{i}=\sum_{j=1}^{6} a_{i j} \sigma_{j}
$$

Recognizing that the total elastic energy for the orthotropic solid is invariant with respect to the coordinate system, the elements $a_{i j}{ }_{j}$ of the material matrix in the $(r, \theta, z)$ coordinate system can be written in terms of $a_{i j}$ of the $(1,2,3)$ coordinate system. That is

$$
\begin{gathered}
a_{i j}^{\prime}=\sum_{m=1}^{6} \sum_{n=1}^{6} a_{m n} q_{m i} q_{n j} \\
i, j=1,2, \ldots .6
\end{gathered}
$$

where $q_{i j}$ represents the direction cosines of the $(1,2,3)$ system with the $(r, \theta, z)$ coordinate system. As shown in Figure 1, the relationship is simply one of rotation of the $(1,2)$ axis to the $(\theta, z)$ axis by an angle of $-\theta_{0}$ in the plane $r=$ constant. The values of $q_{i j}$ for this rotation are listed in Table 1.

In the transformed coordinate system, the constitutive law is

$$
\varepsilon_{i}^{\prime}=\sum_{j=1}^{6} a_{i j}^{\prime} \sigma_{j}^{\prime}
$$

which is expressed in cylindrical coordinates as follows. 


$\left[\begin{array}{l}\varepsilon_{\theta \theta} \\ \varepsilon_{z z} \\ \varepsilon_{r r} \\ r_{z r} \\ \gamma_{\theta r} \\ \gamma_{\theta z}\end{array}\right]=\left[\begin{array}{ccccccc}a_{11}^{\prime} & a_{12}^{\prime} & a_{13}^{\prime} & 0 & 0 & a_{16}^{\prime} & \sigma_{\theta \theta} \\ a_{21}^{\prime} & a_{22}^{\prime} & a_{23}^{\prime} & 0 & 0 & a_{26}^{\prime} & \sigma_{z 2} \\ a_{31}^{\prime} & a_{32}^{\prime} & a_{33}^{\prime} & 0 & 0 & a_{36}^{\prime} & \sigma_{r r} \\ 0 & 0 & 0 & a_{44}^{\prime} & a_{45}^{\prime} & 0 & \sigma_{z r} \\ 0 & 0 & 0 & a_{54}^{\prime} & a_{55}^{\prime} & 0 & \sigma_{\theta r} \\ a_{61}^{\prime} & a_{62}^{\prime} & a_{63}^{\prime} & 0 & 0 & a_{66}^{\prime} & \sigma_{\theta z}\end{array}\right]$

For the types of uniform loading considered here, the shear stresses $\sigma_{\theta r}$ and $\sigma_{z r}$ may be approximated as zero, an assumption consistent with the elastic theory of thin-walled cylinders [6]. From Equation (6), the remaining stress-strain relations are thus

$\left[\begin{array}{l}\varepsilon_{\theta \theta} \\ \varepsilon_{z z} \\ \varepsilon_{r r} \\ r_{\theta z}\end{array}\right]=\left[\begin{array}{llll}a_{11}^{\prime} & a_{12}^{\prime} & a_{13}^{\prime} & a_{16}^{\prime} \\ a_{21}^{\prime} & a_{22}^{\prime} & a_{23}^{\prime} & a_{26}^{\prime} \\ a_{31}^{\prime} & a_{32}^{\prime} & a_{33}^{\prime} & a_{36}^{\prime} \\ a_{61}^{\prime} & a_{62}^{\prime} & a_{63}^{\prime} & a_{66}^{\prime}\end{array}\right]\left[\begin{array}{l}\sigma_{\theta \theta} \\ \sigma_{z z} \\ \sigma_{r r} \\ \sigma_{\theta z}\end{array}\right]$

The elements $\mathbf{a}_{i j}^{\prime}$ of the material matrix of Equation (7) are calculated from Equation (4) with $a_{i j}$ and $q_{i j}$ given. by Equation (1) and Table 1, respectively. The results are as follows*.

Tote that $\phi$ in reference [5] is interpreted as ${ }^{-\theta}$ in Equations
(8)-(17). Also, Equation (9) reflects a correction of the results reported in
$[5]$. 
Table 1. Elements of $q_{i j}$ for axis rotation

\begin{tabular}{|ccccccc|}
\hline$j$ & 1 & 2 & 3 & 4 & 5 & 6 \\
1 & $\cos ^{2} \theta_{0}$ & $\sin ^{2} \theta_{0}$ & 0 & 0 & 0 & $\sin 2 \theta_{0}$ \\
2 & 0 & 0 & 0 & 0 & 0 & $-\sin 2 \theta_{0}$ \\
3 & 0 & 0 & 1 & 0 & 0 & 0 \\
4 & 0 & 0 & 0 & $\cos \theta_{0}$ & $-\sin \theta_{0}$ & 0 \\
5 & 0 & 0 & 0 & $\sin \theta_{0}$ & $\cos \theta_{0}$ & 0 \\
6 & $-\frac{1}{2} \sin 2 \theta_{0}$ & $\frac{1}{2} \sin 2 \theta_{0}$ & 0 & 0 & 0 & $\cos 2 \theta_{0}$ \\
\hline
\end{tabular}




$$
\begin{aligned}
& a_{11}^{\prime}=\frac{1}{E_{11}} \cos ^{4} \theta_{0}+\left(\frac{1}{G_{12}}-\frac{2 v_{12}}{E_{11}}\right) \sin ^{2} \theta_{0} \cos ^{2} \theta_{0}+\frac{1}{E_{22}} \sin ^{4} \theta_{0} \\
& a_{22}^{\prime}=\frac{1}{E_{11}} \sin ^{4} \theta_{0}+\left(\frac{1}{G_{12}}-\frac{2 \nu_{12}}{E_{11}}\right) \sin ^{2} \theta_{0} \cos ^{2} \theta_{0}+\frac{1}{E_{22}} \cos ^{4} \theta_{0} \\
& a_{33}^{\prime}=\frac{1}{E_{33}} \\
& a_{12}^{\prime}=a_{21}^{\prime}=\left(\frac{1}{E_{11}}+\frac{1}{E_{22}}+\frac{2 v_{12}}{E_{11}}-\frac{1}{G_{12}}\right) \sin ^{2} \theta_{0} \cos ^{2} \theta_{0}-\frac{v_{12}}{E_{11}} \\
& a_{23}^{\prime}=a_{32}^{\prime}=-\frac{v_{23}}{E_{22}} \cos ^{2} \theta_{0}-\frac{v_{31}}{E_{33}} \sin ^{2} \theta_{0} \\
& a_{13}^{\prime}=a_{31}^{\prime}=-\frac{\nu_{23}}{E_{22}} \sin ^{2} \theta_{0}-\frac{\nu_{31}}{E_{33}} \cos ^{2} \theta_{0} \\
& a_{66}^{\prime}=\left(\frac{4}{E_{11}}+\frac{4}{E_{22}}+\frac{8 v_{12}}{E_{11}}-\frac{4}{G_{12}}\right) \sin ^{2} \theta_{0} \cos ^{2} \theta_{0}+\frac{1}{G_{12}} \\
& a_{16}^{\prime}=a_{61}^{\prime}=\left(\frac{2}{E_{11}} \cos ^{2} \theta_{0}-\frac{2}{E_{22}} \sin ^{2} \theta_{0}-\left(\frac{1}{G_{12}}-\frac{2 \nu_{12}}{E_{11}}\right)\right. \text {. } \\
& \text { - } \left.\left(\cos ^{2} \theta_{0}-\sin ^{2} \theta_{0}\right)\right] \sin \theta_{0} \cos \theta_{0} \\
& a_{26}^{\prime}=a_{62}^{\prime}=\left[\frac{2}{E_{11}} \sin ^{2} \theta_{0}-\frac{2}{E_{22}} \cos ^{2} \theta_{0}+\left(\frac{1}{G_{12}}-\frac{2 v_{12}}{E_{11}}\right)\right. \text {. } \\
& \text { - } \left.\left(\cos ^{2} \theta_{0}-\sin ^{2} \theta_{0}\right)\right] \sin \theta_{0} \cos \theta_{0} \\
& a_{36}^{\prime}=a_{63}^{\prime}=\left(\frac{-2 v_{31}}{E_{33}}+\frac{2 v_{23}}{E_{22}}\right) \sin \theta_{0} \cos \theta_{0}
\end{aligned}
$$

\section{EVALUATION OF MATERIAL CONSTANTS}

There are seven material constants that appear in Equations (8)-(17), namely $E_{11}, E_{22}, E_{33}, v_{12}, v_{23}, v_{31}$ and $G_{12}$. It is necessary to determine how 
these constants are related to each other and then to devise meaningful tests to measure them. To insure that the strain energy is a single valued function of the strain displacements, the material matrix of Equation (1) must be symmetric. That is

$$
\begin{array}{ll}
E_{11} v_{21}=E_{22} & v_{12} \\
E_{22} & v_{32}=E_{33} v_{23} \\
E_{33} v_{13}=E_{11} & v_{31}
\end{array}
$$

Consider a tensile test for which the specimen's longitudinal axis coincides with the longitudinal or z-axis of an orthotropic cylinder, given that $\theta_{0}=90 \mathrm{deg}$. For a uniform, applied stress $\bar{\sigma}_{z z}$ where $\sigma_{\theta \theta}=\sigma_{r r}=\sigma_{\theta z}=0$, it follows from Equations (7)-(20) that the strains are

$$
\begin{aligned}
& \varepsilon_{\theta \theta}=-\frac{v_{12}}{E_{11}} \bar{\sigma}_{z 2}=-\frac{v_{21}}{E_{22}} \bar{\sigma}_{z 2} \\
& \varepsilon_{z 2}=\frac{1}{E_{11}} \bar{\sigma}_{z z} \\
& \varepsilon_{r r}=-\frac{v_{31}}{E_{33}} \bar{\sigma}_{z 2}=-\frac{v_{13}}{E_{11}} \bar{\sigma}_{z 2}
\end{aligned}
$$

Equation (22) shows that $E_{11}$ is the equivalent of Young's modulus along the 1 axis. Now suppose that for $\theta_{0}=90 \mathrm{deg}$ the orthotropic tube corresponds to a fluted, thin-walled cylindrical column of a homogeneous, isotropic material with Young's modulus $E$ and Poisson's ratio $\nu$. This fluted column test specimen thus exhibits the same stress-strain behavior of the isotropic material in both the 1 (or $z$ ) direction and in the 3 (or $r$ ) direction. The following definitions of the orthotropic constants are consistent with Equations $(21)-(23)$

$$
\begin{aligned}
& E=E_{11}=E_{33} \\
& v=v_{31}=v_{13}=v_{12}
\end{aligned}
$$


Consider a second tensile test for which the specimen's longitudinal axis again corresponds with the z-axis of an orthotropic cylinder, but now let $\theta_{0}=$ 0 deg. Again, the only nonzero stress is the applied, uniform tensile stress $\bar{\sigma}_{z z}$. It follows from Equations $(7)-(20),(24)$ and (25) that

$$
\begin{aligned}
& \varepsilon_{\theta \theta}=-\frac{\nu}{E} \bar{\sigma}_{z Z}=-\frac{\nu_{21}}{E_{22}} \bar{\sigma}_{z 2} \\
& \varepsilon_{z z}=\frac{1}{E_{22}} \bar{\sigma}_{z z} \\
& \varepsilon_{r r}=-\frac{\nu_{23}}{E_{22}} \bar{\sigma}_{z Z}=-\frac{v_{32}}{E} \bar{\sigma}_{z 2}
\end{aligned}
$$

Observing Equation (27), it is seen that the modulus of elasticity in the 2 (or z) direction is $E_{22}$. Now let

$$
\begin{aligned}
& E^{\prime}=E_{22} \\
& v^{\prime}=v_{21}=v_{23}
\end{aligned}
$$

which define respectively the effective Young's modulus and Poisson's ratio for a bellows, consistent with the assumption that $\sigma_{\theta \theta}=\sigma_{\mathrm{rr}}$ in this tensile test. It follows, using Equations (24), (25), (29), (30), that Equations (18)-(20) are satisfied if

$$
\nu=\nu_{32}, E \nu^{\prime}=E^{\prime} \nu
$$

In a third test, a pure shear stress $\sigma_{\theta z}$ is applied to an orthotropic cylinder for which $\theta_{0}=0$. This corresponds to the application of a pure torque that rotates a bellows about its longitudinal axis. As for the previous two tests, the cylinder is orthotropic only because of its corrugations, since it is constructed of an isotropic material with elastic constants $E, v$ and shear modulus $G$. Torsion tests on such a bellows have shown that [8]

$$
\gamma_{\theta z}=\frac{\sigma_{\theta z}}{G}
$$

which indicates that the rotation and shearing stress are those predicted by 
the theory of thin-walled tubes without corrugations. When Equation (32) is compared with the stress-strain law given by Equations (7)-(17) in this case, it is seen that

$$
G=G_{12}
$$

In summary, there are four independent material constants needed to describe the stress-strain behavior of a cylinder with corrugated walls made of an isotropic material, but modeled as an orthotropic, smooth-walled tube. The stiffest direction (modulus E) follows the corrugation lines forming constant angle helices. Orthogonal to the helices is the weakest direction (modulus $\left.E^{\prime}\right)$. The independent material constants are $E, E^{\prime}, G$ and $v$. Given uniform stresses $\sigma_{\theta \theta}, \sigma_{z z}, \sigma_{r r}$, and $\sigma_{\theta z}$, the uniform strains are calculated from Equation (7). Using Equations (24), (25), (29-31) and (33), Equations (8)-(17) give the elements of the material matrix as follows.

$$
\begin{aligned}
& a_{11}^{\prime}=\frac{1}{E} \cos ^{4} \theta_{0}+\left(\frac{1}{G}-\frac{2 \nu}{E}\right) \sin ^{2} \theta_{0} \cos ^{2} \theta_{0}+\frac{1}{E} \sin ^{4} \theta_{0} \\
& a_{22}^{\prime}=\frac{1}{E} \sin ^{4} \theta_{0}+\left(\frac{1}{G}-\frac{2 v}{E}\right) \sin ^{2} \theta_{0} \cos ^{2} \theta_{0}+\frac{1}{E} \cos ^{4} \theta_{0} \\
& a_{33}^{\prime}=\frac{1}{E} \\
& a_{12}^{\prime}=a_{21}^{\prime}=\left(\frac{1}{E}+\frac{1}{E}+\frac{2 v}{E}-\frac{1}{G}\right) \sin ^{2} \theta_{0} \cos ^{2} \theta_{0}-\frac{v}{E} \\
& a_{23}^{\prime}=a_{32}^{\prime}=-\frac{v}{E} \\
& a_{13}^{\prime}=a_{31}^{\prime}=-\frac{v}{E} \\
& a_{66}^{\prime}=\left(\frac{4}{E}+\frac{4}{E}+\frac{8 \nu}{E}-\frac{4}{G}\right) \sin { }^{2} \theta_{0} \cos ^{2} \theta_{0}+\frac{1}{G} \\
& a_{16}^{\prime}=a_{61}^{\prime}=\left[-\frac{2}{E} \sin { }^{2} \theta_{0}+\frac{2}{E} \cos ^{2} \theta_{0}-\left(\frac{1}{G}-\frac{2 \nu}{E}\right) \cdot\right. \\
& \left.\quad \cdot\left(\cos ^{2} \theta_{0}-\sin \theta_{0}\right)\right] \sin _{0} \theta_{0} \cos \theta_{0}
\end{aligned}
$$




$$
\begin{aligned}
a_{26}^{\prime}= & a_{62}^{\prime}=\left[-\frac{2}{E} \cos ^{2} \theta_{0}+\frac{2}{E} \sin ^{2} \theta_{0}+\left(\frac{1}{G}-\frac{2 v}{E}\right) \cdot\right. \\
& \left.\cdot\left(\cos ^{2} \theta_{0}-\sin ^{2} \theta_{0}\right)\right] \sin \theta_{0} \cos \theta_{0} \\
a_{36}^{\prime}= & a_{63}^{\prime}=0
\end{aligned}
$$

These results can be used to calculate the strains, displacements and rotations of the cylinder for the special cases of loading discussed below.

\section{EQUILIBRIUM AND COMPATIBILITY}

As shown in Figure 1, the thin-walled cylinder is subjected to three types of uniform loads: an internal pressure $p$, a longitudinal load $P$ centered on the $z$-axis, and a pure torque $T$ causing rotation about the z-axis. Since there are no boundary or edge constraints, the resulting uniform stresses may be derived using equilibrium conditions and elementary methods. In terms of the mean radius $R_{0}$ and shell thickness $t_{0}$, which are essentially constant during loading, the stresses are:

$$
\begin{gathered}
\sigma_{\theta \theta}=\frac{P R_{0}}{t_{0}} \\
\sigma_{\theta \theta}=\frac{P}{2 \pi R_{0} t_{0}}+\frac{P R_{0}}{2 t_{0}} \\
\sigma_{\theta \theta}=\frac{T}{2 \pi R_{0}^{2} t_{0}}
\end{gathered}
$$

Consistent with the thin-wall assumption, the radial stress is negligible, or $\sigma_{r r}=0[6]$.

Since these stresses and their corresponding strains, as given by Equations (7) and (34-43), are all uniform, they are all independent of the cylindrical coordinates $(r, \theta, z)$. Thus the 81 St. Venant strain compatibility conditions are automatically satisfied [7]. 


\section{STRAIN-DISPLACEMENT RELATIONS}

The general strain-displacement equations in polar-cylindrical coordinates given in [7] are simplified as follows to express the condition that there are no variations in displacement in the $\theta$ or circumferential direction.

$$
\begin{aligned}
& \varepsilon_{r r}=\frac{\partial U_{r}}{\partial r} \\
& \varepsilon_{\theta \theta}=\frac{1}{r} \frac{\partial U_{\theta}}{\partial \theta}+\frac{U_{r}}{R}=\frac{U_{r}}{r} \\
& \varepsilon_{z z}=\frac{\partial U_{z}}{\partial z} \\
& \gamma_{\theta z}=\frac{\partial U_{\theta}}{\partial z}+\frac{1}{r} \frac{\partial U_{z}}{\partial \theta}=\frac{\partial U_{\theta}}{\partial z}
\end{aligned}
$$

Here, $U_{r}, U_{\theta}$, and $U_{z}$ are respectively the displacements in the $r, \theta$ and $z$ directions.

Following a procedure in [3], these relationships can be integrated in terms of three functions $f_{1}(r, z), f_{2}(z)$ and $f_{3}(z)$ which are to be determined. From Equation (47):

$$
U_{r}=r \varepsilon_{r r}+f_{1}(r, z)
$$

It follows from Equations (48) and (51) that:

$$
f_{l}(r, z)=r\left(\varepsilon_{\theta \theta}-\varepsilon_{r r}\right)
$$

From the last two equations, then,

$$
U_{r}=r \varepsilon_{\theta \theta}=R_{0} \varepsilon_{\theta \theta}
$$

where $r=R_{0}$ for a thin-walled cylinder.

From Equation (49):

$$
U_{z}=z \varepsilon_{z z}+f_{2}(z)
$$

where the second function of integration, $f_{2}(z)$, is independent of $r$ since the cylinder wall is very thin and any variations across this thickness have no significant effect on the longitudinal deformation. Further, by imposing the 
condition of no rigid body motion, where $U_{z}=0$ at $z=0$, and noting that $U_{z}$ can be at most a linear function of $z$, then it is apparent that $f_{2}(z)=0$. Thus Equation (54) thus yields the position $\xi=z+U_{z}$ of a material point originally at point $z$ measured from one end of the cylinder, or

$$
\xi=\left(1+\varepsilon_{z z}\right) z
$$

The circumferential displacement is obtained by integrating Equation (50) and then using Equation (55). That is

$$
U_{\theta}=\xi \gamma_{\theta z}+f_{3}(z)
$$

Here the function of integration is again independent of $r$, which is consistent with the thin-wall assumption. By imposing the condition of no rigid body rotation where $U_{\theta}=0$ at $z=0$, and noting that $U_{\theta}$ can at most be a linear function in $z$, it is evident that $f_{3}(z)=0$. Thus $U_{\theta}=\xi \gamma_{\theta z}$.

In summary, the radial, circumferential and longitudinal displacements are given in terms of the strains, the mean radius, and the 2 -coordinate only, or

$$
U_{r}=R_{0} \varepsilon_{\theta \theta} ; U_{\theta}=\left(1+\varepsilon_{z z}\right) z \gamma_{\theta z} ; U_{z}=z \varepsilon_{z z}
$$

It is noted that $U_{\theta}$ and $U_{z}$ are the same displacements assumed [2] for the solid orthotropic cylinder rotating about the z-axis.

A further displacement of practical interest is the angle of rotation $\phi$ for a cross section of the cylinder at the coordinate $z$. Within the limits of linear theory, $\phi=U_{\theta} / R_{0}$. Thus, from Equation (57):

$$
\phi=\frac{z}{R_{0}}\left(1+\varepsilon_{z z}\right) \gamma_{\theta z}
$$

\section{PARAMETRIC STUDIES}

All of the calculations for the parametric studies that follow are based on Equation (7), the stresses of Equations (44)-(46), and the elements of the material matrix given by Equations $(34)-(43)$. The specific values of $E / G=3$ 
and $v=0.5$ were chosen since they are characteristic of the polymeric and rubber-like materials to be used in future applications.

Effects of Loading without Constraint. In the first series of parametric studies, the separate effects of each loading, $P, P$ and $T$ on the homogeneous strains $\gamma_{\theta z}$ and $\varepsilon_{z z}$ were investigated for an unconstrained cylinder. The goal was to determine peak strains as a function of the appropriate nondimensional system parameters.

The three nondimensional parameters involving the shear strain $\gamma_{\theta z}$ are defined by the ordinates of Figures 2 through 4, in which the only nonzero load is $P, P$ and $T$, respectively. For the isotropic case $\left(E / E^{\prime}=1\right)$, all three of these figures show no shear strain variations with the helix angle $\theta_{0}$, a result that could be anticipated. For $p$ loading only (Figure 2) and for $P$ loading only (Figure 3) the negative ordinates indicate that $\gamma_{\theta z}$ is negative, or that the cylinder "unwinds" because of orthotropy $\left(E / E^{\prime}>1\right)$. For $T$ loading only (Figure 4), the positive ordinate indicates that the cylinder "winds up" if $T$ is applied as shown in Figure 1. The interesting result is that the peak value of each respective shear strain parameter occurs at a distinctly different value of $\theta_{0}$. That is:

$$
\begin{aligned}
& \text { peak }\left(\frac{E t_{0} \gamma_{\theta z}}{p R_{0}}\right) \text { occurs for } \theta_{0}=53 \mathrm{deg} \\
& \text { peak }\left(\frac{E t_{0} R_{0} \gamma_{\theta z}}{p}\right) \text { occurs for } \theta_{0}=30 \mathrm{deg} \\
& \text { peak }\left(\frac{E t_{0} R_{0}^{2} \gamma_{\theta z}}{T}\right) \text { occurs for } \theta_{0}=45 \mathrm{deg}
\end{aligned}
$$

For each load type, there is no shift in these respective values of $\theta_{0}$ for $1<E / E^{\prime}<100$. 


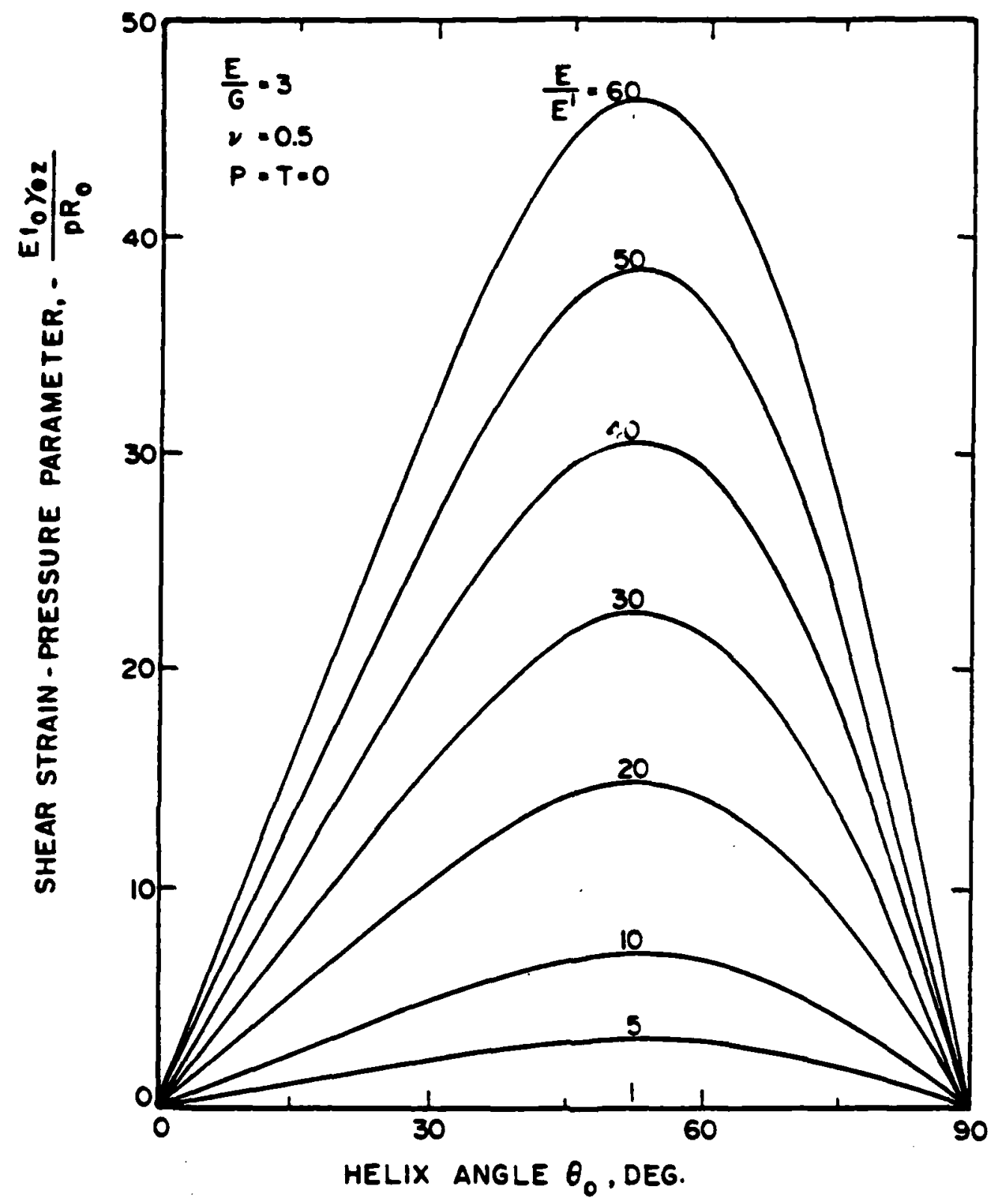

Figure 2 Unrestrained twisting due to internal pressure 


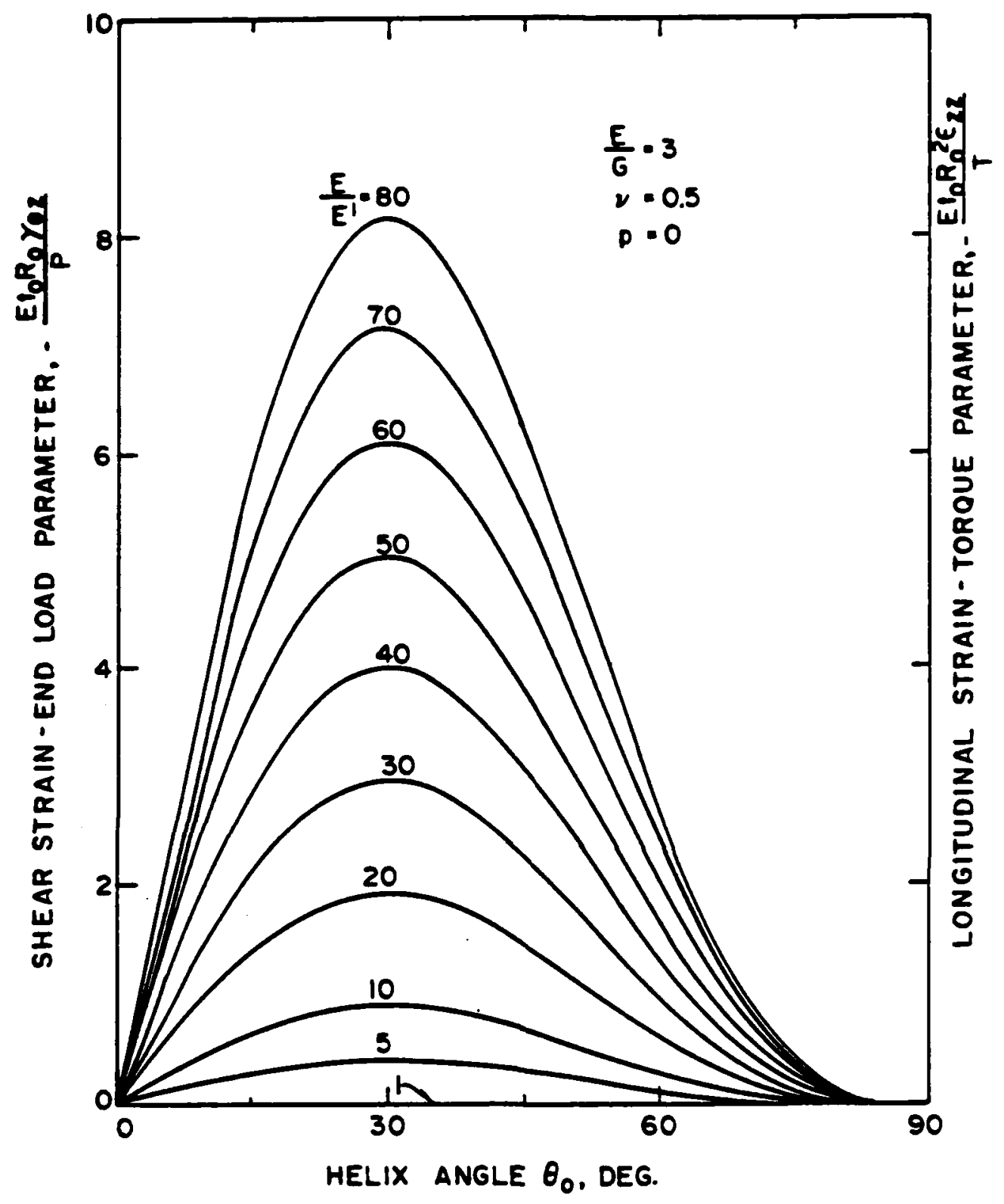

Figure 3 Unrestrained twisting due to longitudinal load (left ordinate) and unrestrained longitudinal strain due to pure torque (right ordinate) 


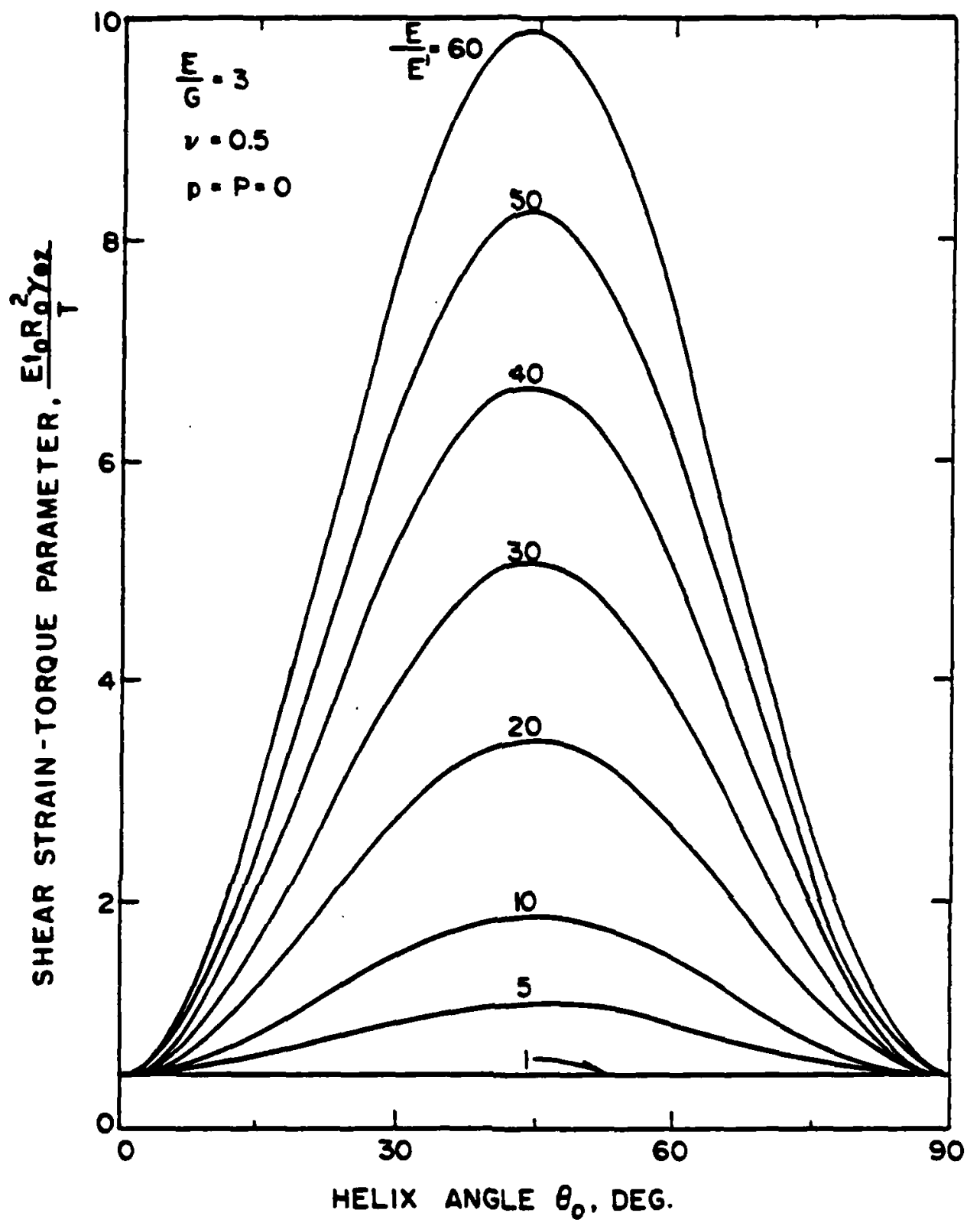

Figure $4 \quad$ Unrestrained twisting due to a pure torque

84 
The three nondimensional parameters involving the longitudinal strain $\varepsilon_{z z}$ are defined by the ordinates of Figures 5, 6 and 3 (right ordinate), given that $P, P$ and $T$ are the only nonzero loads, respectively. For the isotropic case $\left(E / E^{\prime}=1\right), \varepsilon_{z z}$ is zero for two load cases: with the $p$ load as a direct consequence of choosing $v$ as 0.5 , and with the $T$ load as expected from elementary solutions. With orthotropy, the peak values of the longitudinal strain parameters are as follows:

$$
\begin{aligned}
& \text { peak }\left(\frac{E t_{0} \varepsilon z z}{p R_{0}}\right) \text { occurs for } \theta_{0}=0 \\
& \text { peak }\left(\frac{E t_{0} R_{0} \varepsilon z z}{p}\right) \text { occurs for } \theta_{0}=0 \\
& \text { peak }\left(\frac{E t_{0} R_{0}^{2} \varepsilon z z}{T}\right) \text { occurs for } \theta_{0}=30 \text { deg }
\end{aligned}
$$

From Figures 5 and 6 it is observed that the values of these parameters are only somewhat depressed for $0<\theta_{0}<15 \mathrm{deg}$, but that this depression increases more rapidly as E/E' increases. From Figure 3 (right ordinate) the longitudinal strain is seen to be negative, indicating the tendency of the orthotropic cylinder to shorten as it winds up under a positive torsion load.

Effects of Radial and End Constraint. While the studies above dealt with strain behavior for loadings applied one at a time, one can imagine a multitude of practical cases involving combined loads. Consider now one such case where an orthotropic cylinder is required to operate in close proximity with other mechanical parts. To avoid longitudinal and radial or circumferential expansions when pressurized, suppose that the unloaded orthotropic cylinder just fits within the confines of a rigid, closed-end tube. Under loading, then:

$$
\varepsilon_{z z}=\varepsilon_{\theta \theta}=0
$$




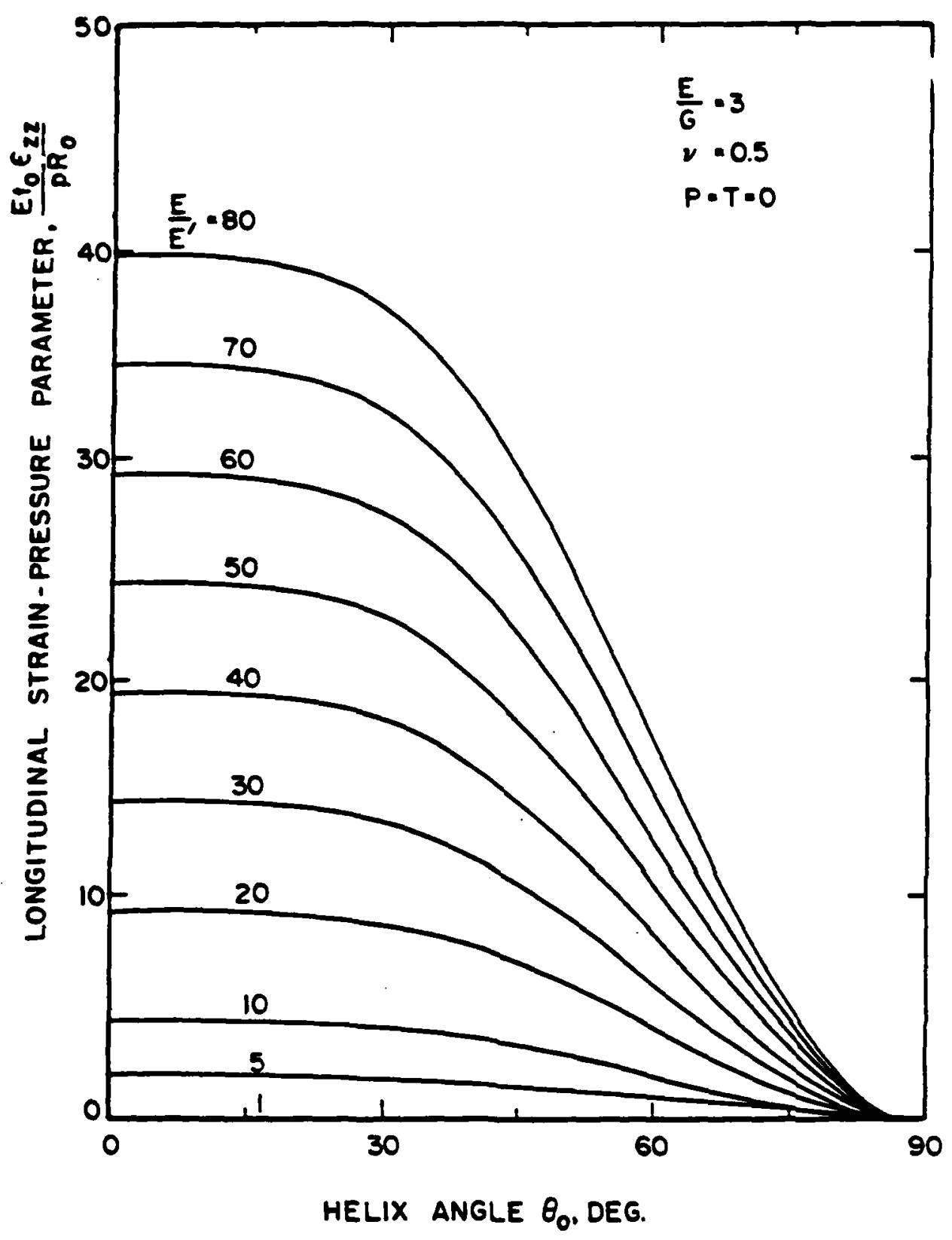

Figure 5

Unrestrained longitudinal strain due to internal pressure 


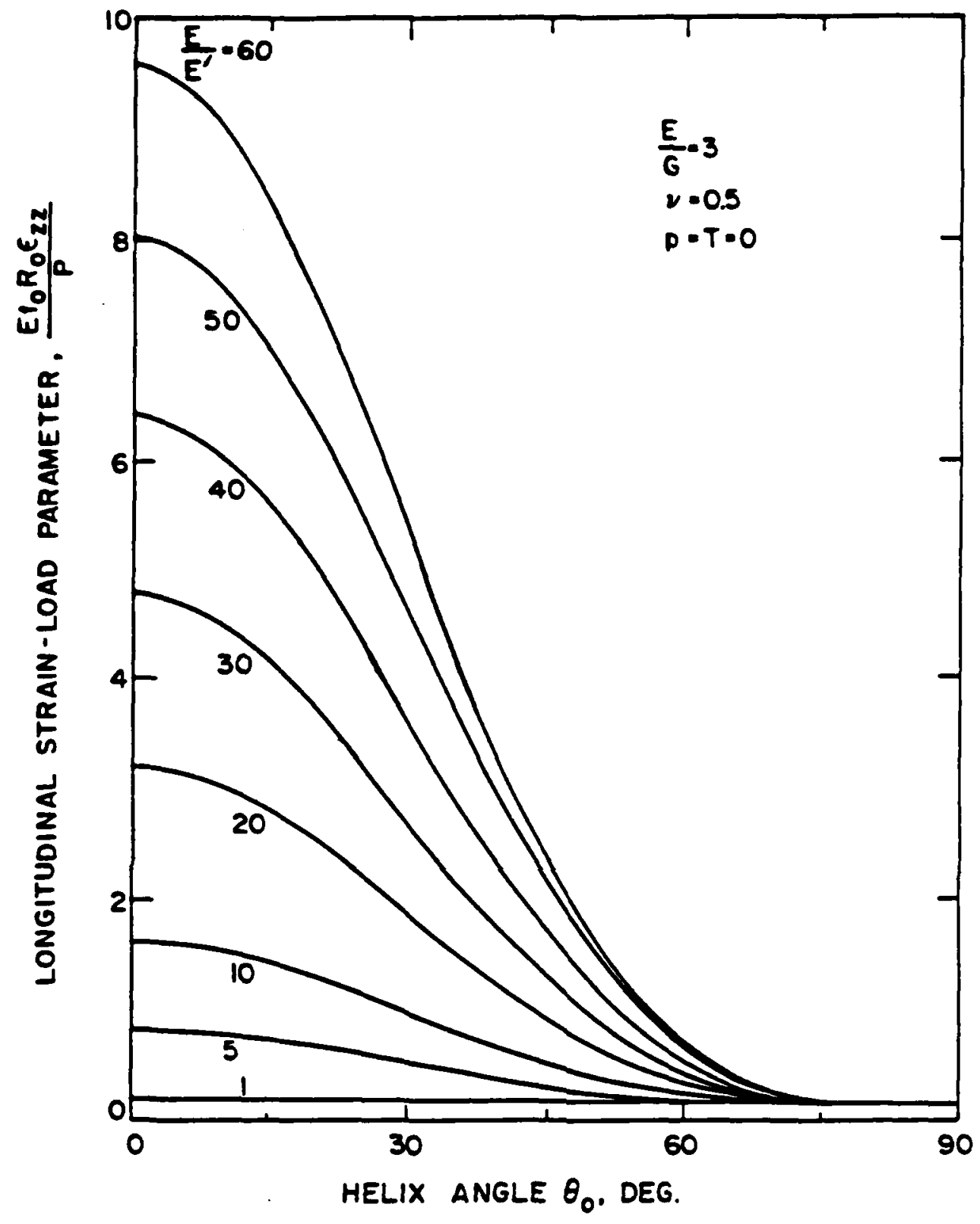

Figure 6

Unrestrained longitudinal strain due to longitudinal load 
The confining tube has frictionless walls where its design allows for the application of a torque $T$ to the orthotropic cylinder. The confining tube does not inhibit torsion. From Equations (7) and (44-46), the two constraints of Equation (59) become:

$$
\begin{aligned}
& \left(a_{12}^{\prime}+\frac{1}{2} a_{22}^{\prime}\right) \frac{p R_{0}}{t_{0}}+a_{22}^{\prime} \frac{p}{2 \pi R_{0} t}+a_{26}^{\prime} \frac{T}{2 \pi R_{0}^{2} t_{0}}=0 \\
& \left(a_{11}^{\prime}+\frac{1}{2} a_{12}^{\prime}\right) \frac{p R_{0}}{t_{0}}+a_{12}^{\prime} \frac{p}{2 \pi r_{0} t}+a_{16}^{\prime} \frac{T}{2 \pi R_{0}^{2} t_{0}}=0
\end{aligned}
$$

The compatible equation relating the loadings $P$ and $T$ is found by eliminating $P$ between Equations (60) and (61), or

$$
\left.\frac{T}{p R_{0}^{3}}=2 \pi \frac{a_{12}^{\prime} a_{12}^{\prime}-a_{11}^{\prime} a_{22}^{\prime}}{a_{16}^{\prime} a_{22}^{\prime}-a_{12}^{\prime} a_{26}^{\prime}}\right)=s
$$

The pressure-longitudinal load parameter is found by eliminating $T$ between Equations (60) and (61). In terms of $S$ of Equation (62), this is

$$
\frac{p}{p R_{0}^{2}}=-2 \pi\left(\frac{a_{12}^{\prime}}{a_{22}^{\prime}}+\frac{1}{2}+\frac{a_{26}^{\prime}}{a_{22}^{\prime}} s\right)
$$

The results of Equations (62) and (63) are shown in Figures 7 and 8. Figure 7 shows that the pressure-torque parameter, the inverse of Equation (62), has a peak value at $\theta_{0}=50 \mathrm{deg}$ for all $E / E^{\prime}>1$. For an imposed torque, this gives the maximum required cylinder pressure for full cylinder confinement. Figure 8 (the right ordinate) shows that the end load-pressure parameter defined by Equation $(63)$ is independent of $E / E^{\prime}$ if that ratio exceeds unity. For a fixed pressure level $p$, the compatible end load $P$ always decreases with $\theta_{0}$ to maintain full cylinder confinement. It is noted that the isotropic cylinder is not shown in Figure 8 (the right ordinate) since the only 


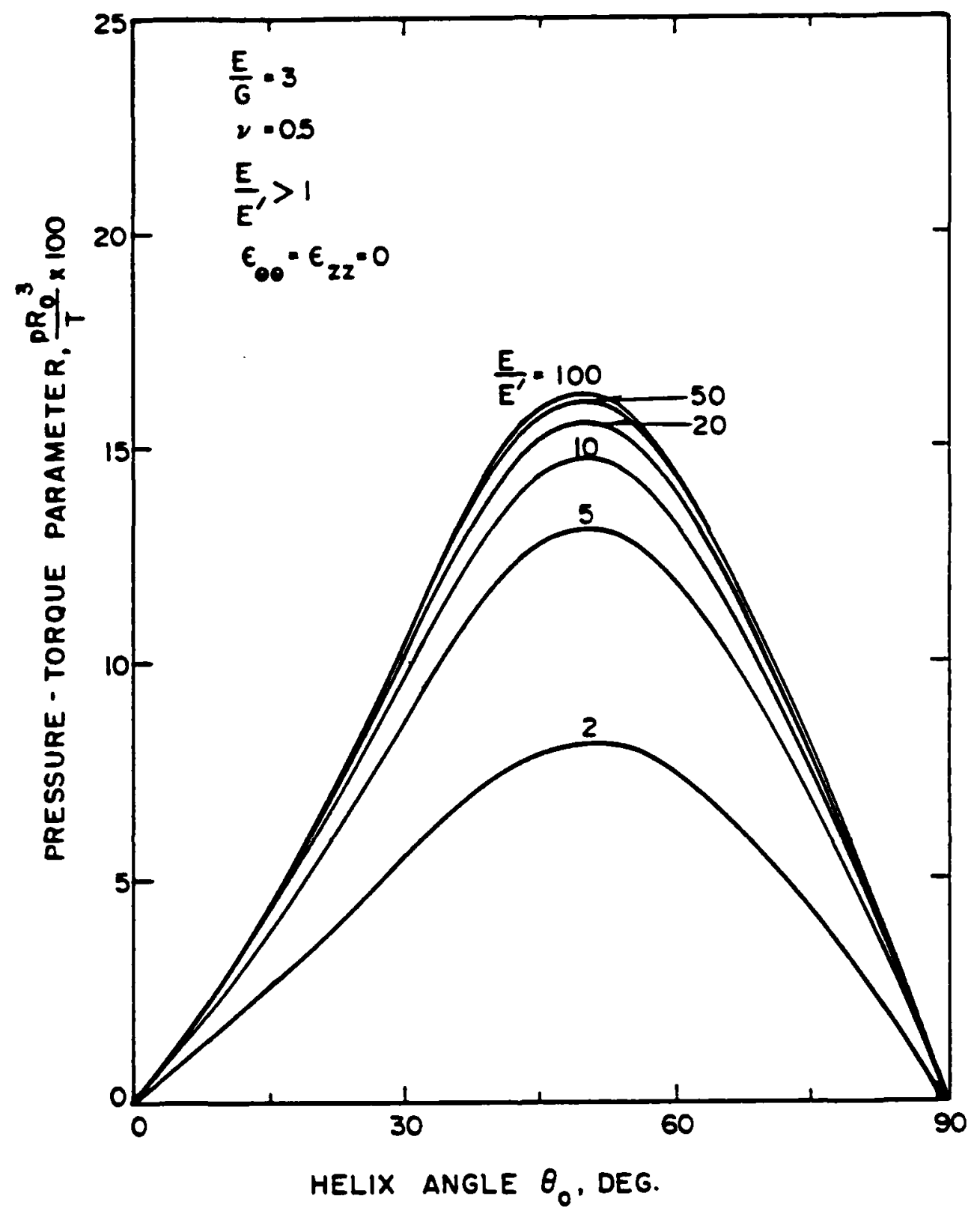

Figure 7 Pressure-torque behavior for the fully confined cylinder 


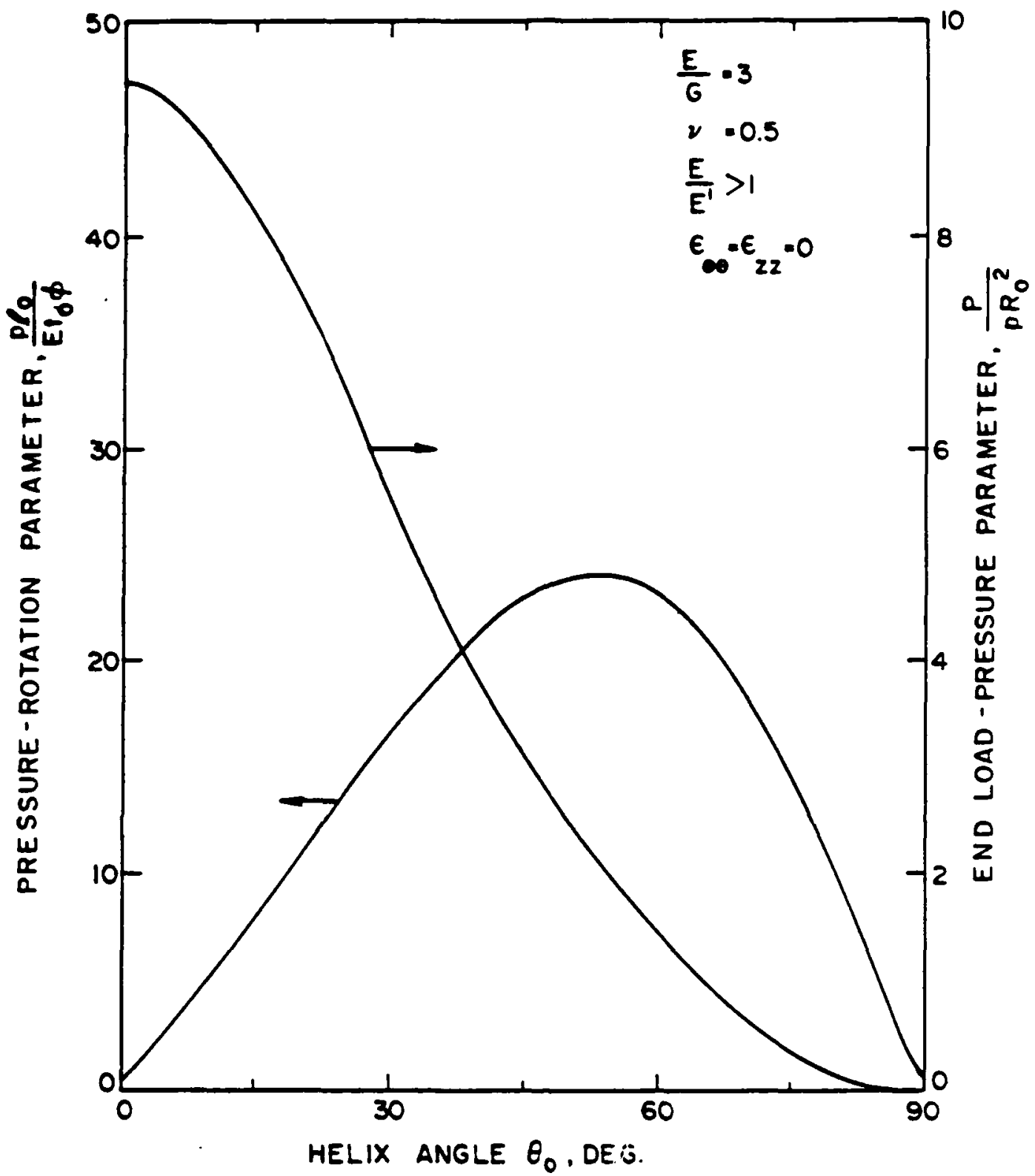

Figure 8

Rotation (left ordinate) and longitudinal load (right ordinate) for a pressurized and fully confined cylinder 
Way that full cylinder confinement can be met for $E / E^{\prime}=1$ is that $P=P=0$. For this latter set of conditions, it is recalled that a nonzero value for $T$ always implies full cylinder confinement; that is, Equation (59) is satisfied. Using the results of Equations (62) and (63) it is possible to calculate the helix angle that will minimize the rotation (minimize $\gamma_{\theta z}$ ) at a given pressure level. The conclusion in this case, based on the results of figure 8 , left ordinate, is that $\theta_{0}=53 \mathrm{deg}$ will produce a minimum for all $\mathrm{E} / \mathrm{E}^{\prime}>1$. Effects of Torsional and End Constraint. Consider now the special case where the orthotropic cylinder is fully restrained from rotation and also from longitudinal displacement. That is

$$
Y_{\theta Z}=\varepsilon_{Z 2}=0
$$

With Equations (7) and (34)-(46) these two constraint equations are:

$$
\begin{aligned}
& \left(a_{12}^{\prime}+\frac{1}{2} a_{22}^{\prime}\right) \frac{p R_{0}}{t_{0}}+a_{22}^{\prime} \frac{p}{2 \pi R_{0} t_{0}}+a_{26}^{\prime} \frac{T}{2 \pi R_{0}^{2} t_{0}}=0 \\
& \left(a_{16}^{\prime}+\frac{1}{2} a_{26}^{\prime}\right) \frac{p R_{0}}{t_{0}}+a_{26}^{\prime} \frac{p}{2 \pi R_{0} t_{0}}+a_{66}^{\prime} \frac{T}{2 \pi R_{0}^{2} t_{0}}=0
\end{aligned}
$$

By eliminating $P$ between Equations (65) and (66), the torque-pressure parameter is deduced as:

$$
\frac{T}{P R_{0}^{3}}=-2 \pi\left(\frac{a_{16}^{\prime} a_{22}^{\prime}-a_{26}^{\prime} a_{12}^{\prime}}{a_{22}^{\prime} a_{66}^{\prime}-a_{26}^{\prime} a_{26}^{\prime}}\right)=S^{\prime}
$$

The compatible end load-pressure parameter is found by eliminating $T$ between the same two equations. In terms of $S^{\prime} .0$ Equation (67), this is

$$
\frac{P}{P R_{0}^{2}}=-2 \pi\left(\frac{a_{12}^{\prime}}{a_{22}^{\prime}}+\frac{1}{2}+\frac{a_{26}^{\prime}}{a_{22}^{\prime}} S^{\prime}\right)
$$

The results of these studies are shown in Figures 9 and 10 . For a constant pressure $p$ and a fixed modulus ratio $E / E^{\prime}$, it is observed that both 


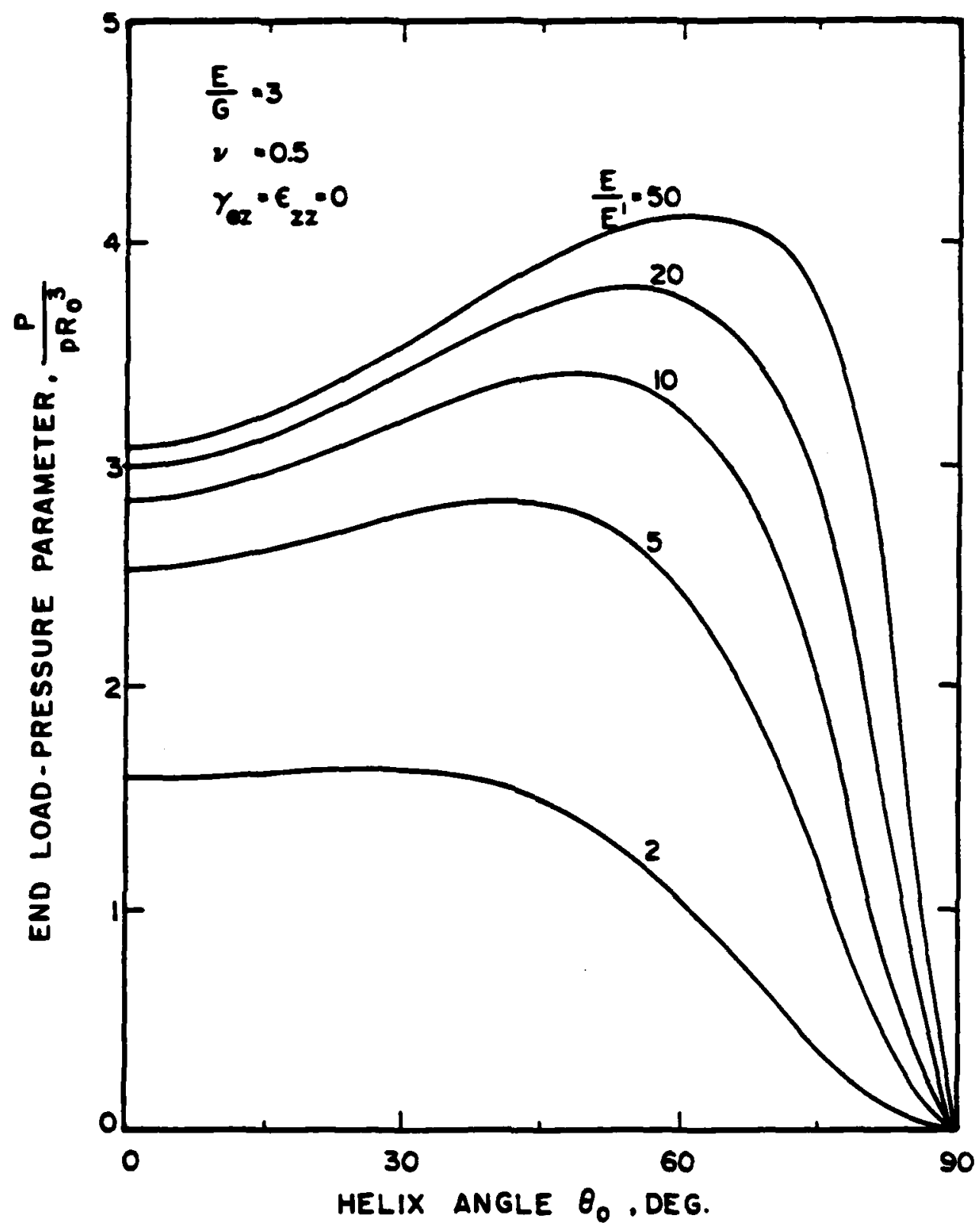

Figure 10 End load required to supress longitudinal strain and rotation in a pressurized cylinder 
the applied torque $T$ and end load $P$ reach peaks that are highly dependent on the helix angle. For instance, if the orthotropic cylinder were to be used as an efficient torque-exerting device, we would pick $\theta_{0}=60 \mathrm{deg}$ if $E / E^{\prime}=2$, but would pick $\theta_{0}=80 \mathrm{deg}$ if $E / E^{\prime}=50$. However, if this cylinder were to be used as a longitudinal or end loading device, we would choose $\theta_{0}=62 \mathrm{deg}$ for $E / E^{\prime}=$ 50. These résults are contingent, of course, on the requirement that all rotations and longitudinal motions be completely supressed.

There are three observations for this case where $\theta_{0}=90 \mathrm{deg}$ and $p>0$. First, no end load is required for the longitudinal strain to vanish, which is a direct consequence of having chosen $v=0.5$. Second, no torque is required and $\gamma_{\theta z}$ is automatically zero, a result that agrees with intuition. Third, a straightforward calculation of the circumferential strain reveals that $\varepsilon_{\theta \theta}$ reaches its peak value when $\theta_{0}=90 \mathrm{deg}$, for all E/E' $>1$.

\section{DESIGN EXAMPLES}

The following examples show the utility of the above parametric studies in the design of special purpose configurations. The finite length cylinders are assumed to be fitted with end caps that have negligible radial stiffness so that homogeneous stresses are maintained throughout. The material and geometric properties common to all three design examples discussed below are as follows.

$$
\begin{array}{ll}
E=1.5 \times 10^{7} \mathrm{~N} / \mathrm{m}^{2}, & E^{\prime}=1.5 \times 10^{6} \mathrm{~N} / \mathrm{m}^{2}, \\
G=5 \times 10^{6} \mathrm{~N} / \mathrm{m}^{2}, & v=0.5, \\
t_{0}=1.5 \mathrm{~mm}, R_{0}=15 \mathrm{~mm}, & \ell_{0}=50 \mathrm{~mm} .
\end{array}
$$

In the first design, $\theta_{0}=30 \mathrm{deg}$ and the maximum allowable internal pressure is $p=1.9 \times 10^{5} \mathrm{~N} / \mathrm{m}^{2}$. What is the maximum torque $T$ that such a cylinder can exert to loosen a bolt, for instance? With $E / E^{\prime}=10$, the value 
In the third design, the applied torque and the longitudinal strain are zero. What value of $\theta_{0}$ will produce the maximum cylinder rotation at an arbitrary internal pressure? In this case, $\mathrm{Y}_{\theta 2}$ is the sum of two components: that due to the internal pressure (Figure 2) and that due to the longitudinal load (Figure 3, left ordinate). In terms of the ordinates $r_{2}$ and $r_{3}$ of these respective Figures, as well as the ordinates $Y_{5}$ and $Y_{6}$, the total shear strain reduces to:

$$
\gamma_{\theta z}=\left(-Y_{2}+\frac{Y_{3} Y_{5}}{Y_{6}}\right) \frac{p R_{0}}{E t_{0}}
$$

In Equation (70), the value of $P$ was eliminated from $Y_{3}$ by the multiple $Y_{5} / Y_{6}$. Using a trial and error procedure, the peak value of the bracket term in Equation (70) was calculated as -4.94 , for which $\theta_{0}=67$ deg. If the internal pressure is $p=3.8 \times 10^{4} \mathrm{~N} / \mathrm{m}^{2}$, then the corresponding shear strain is $\gamma_{\theta z}=$ $-0.125 \mathrm{rad}$; and the rotational displacement and angle of rotation at $z=2_{0}$, calculated from Equations (57) and (58), are $U_{\theta}=-6.25 \mathrm{~mm}$ and $\phi=-0.42 \mathrm{rad}$, respectively.

\section{SUMMARY AND CONCLUSIONS}

Within the limits of linear theory, the deformation behavior of uniformly stressed, orthotropic cylindrical shells is described by several nondimensional parameters involving four independent material constants $E, E^{\prime}, G, v$, and three loads: internal pressure, longitudinal load and pure torque. It is assumed that buckling is absent and that the cylinder is sufficiently constrained so that the cylindrical shape is always maintained. As shown in Figure 1, the principal directions of orthotropy follow constant angle helices. The more important findings of the parametric studies, unique to this type of orthotropy, are summarized as follows. 
1. Longitudinal loading $P$ and/or internal pressure $p$ effects twisting about the longitudinal axis.

2. For P loading only, maximum twisting deformation occurs for an orthotropy angle of $\theta_{0}=30 \mathrm{deg}$; but for p loading only, this occurs when $\theta_{0}=53 \mathrm{deg}$.

3. For a pure torque $T$, maximum twisting deformation occurs when $\theta_{0}=45$ deg.

4. A pure torque $T$ produces a change in cylinder length, which is not the case for isotropic cylinders.

5. The cylindrical strains $Y_{\theta z}, \varepsilon_{\theta \theta}$ and $\varepsilon_{z z}$ are all amplified for a given set of loads $(P, P, T)$ as the magnitude of the orthotropy increases, that is, as E/E' increases, given that the geometry and other material constants remain fixed.

6. The maximum longitudinal strain $\varepsilon_{z z}$ for $P$ and $p$ loading occurs for $\theta_{0}$ $=0$; but $\varepsilon_{z z}$ is maximum for $T$ loading when $\theta_{0}=30 \mathrm{deg}$.

7. The design examples show that the proper selection of cylinder orthotropy $\left(\theta_{0}\right.$ and $\left.E / E^{\prime}\right)$ can lead to optimal deformations or load-carrying capacity. Such designs, however, are highly sensitive to both the type of loading and strain constraint conditions .

The results of these studies are important to the design of versatile and efficient pressure-controlled actuators made of rubber-like materials that can sustain relatively high strains. Orthotropy may be achieved by corruyations of the wall. Robotics is a logical field of application for such actuators. 
NOMENCLATURE

E

$E^{\prime}$

Ei

G

$\mathbf{G}_{\mathbf{j}}$

P

$R_{0}$

$T$

$Y_{k}$

$a_{i j}$

$\mathbf{a}_{\mathbf{i j}}^{\prime}$

$f_{1}(r, z)$

$f_{2}(z), f_{3}(z)$ Functions of integration

so

p

$\mathbf{q} \mathbf{j}$

$r, \theta, z$

$t_{0}$

$U_{r}$

$\mathbf{U}_{\mathbf{Z}}$

$\mathbf{U}_{\boldsymbol{\theta}}$

క $(i, j)=1,2,3, i \neq j$

Longitudinal load

Mean radius of cylinder

Applied end torque $(i, j)=1,2, \ldots .6$ $(i, j)=1,2, \ldots 6$

Function of integration

Length of cylinder

Internal pressure axis, $(i, j)=1,2, \ldots .6$

Cylindrical coordinates

Wall thickness

Radial displacement
Young's modulus in strong direction of orthotropy

Young's modulus in weak direction of orthotropy

Modulus of elasticity in $i$ direction where $i$ is one of the orthotropic axes, $i=1,2,3$

Shear modulus of an isotropic material with Young's modulus $E$

Shear modulus in $i-j$ plane, where $i$ and $j$ are orthotropic axes,

Value of ordinate for Figure $k, k=2,3, \ldots .6$

elastic constants in orthotropic coordinate system

Elastic constants in cylinder coordinate system

Direction cosine terms relating the $(1,2,3)$ axis to $(r, \theta, z)$

Longitudinal displacement

Circumferential displacement

Longitudinal coordinate of material point where $\xi=z$ at $\varepsilon_{Z Z}=0$ 


\begin{tabular}{|c|c|}
\hline$\gamma_{\mathbf{i j}}$ & $\begin{array}{l}\text { Shear strains in } i-j \text { plane in orthotropic coordinate system, } \\
(i, i)=1,2,3, i \neq j\end{array}$ \\
\hline$r_{2 r}$ & Shear strain in $z-r$ plane \\
\hline$Y_{\theta r}$ & Shear strain in $\theta-r$ plane \\
\hline$Y_{\theta z}$ & Shear strain in $\theta-z$ plane \\
\hline$\varepsilon_{\mathbf{i}}$ & $\begin{array}{l}\text { Strains in orthotropic coordinate system, single index } \\
\text { notation } i=1, \ldots .6\end{array}$ \\
\hline$\varepsilon_{i}^{\prime}$ & $\begin{array}{l}\text { Strains in cylinder coordinate system, single index notation } \\
i=1, \ldots .6\end{array}$ \\
\hline$E_{i j}$ & $\begin{array}{l}\text { Strains in orthotropic coordinate system, double index } \\
\text { notation } i=1,2,3\end{array}$ \\
\hline$\varepsilon_{r r}$ & Strain in radial direction \\
\hline$\varepsilon_{\mathbf{Z Z}}$ & Strain in longitudinal direction \\
\hline$\varepsilon_{\theta \theta}$ & Strain in circumferential direction \\
\hline$\theta_{0}$ & $\begin{array}{l}\text { Angle between strong axis of orthotropy and circumferential } \\
\text { direction on cylinder }\end{array}$ \\
\hline$v$ & Poisson's ratio of isotropic material with Young's modulus $E$ \\
\hline$v_{\mathbf{i j}}$ & $\begin{array}{l}\text { Ratio of extensional strain in } i \text { direction to extensionai } \\
\text { strain in } j \text { direction for orthotropic coordinate system, } \\
(i, j)=1,2,3, i \neq j\end{array}$ \\
\hline$\sigma_{i}$ & $\begin{array}{l}\text { Stresses in orthotropic coordinate system, } i=1,2, \ldots 6 \\
\text { Stresses in cylinder coordinate system, } i=1,2, \ldots .6\end{array}$ \\
\hline$\sigma_{i j}$ & $\begin{array}{l}\text { Stresses in orthotropic coordinate system, }(i, j)=1,2,3 \\
\text { Normal stress in radial direction }\end{array}$ \\
\hline$\sigma_{z r}$ & Shear stress in $z-r$ plane \\
\hline$\sigma_{22}$ & Normal stress in longitudinal direction \\
\hline$\sigma_{\theta r}$ & Shear stress in $\theta-r$ plane \\
\hline$\sigma_{\theta z}$ & Shear stress in $\theta-z$ plane \\
\hline$\sigma_{\theta \theta}$ & Normal stress in circumferential direction \\
\hline$\phi$ & Angle of rotation of cross-section \\
\hline
\end{tabular}




\section{REFERENCES}

1. Wilson, J. F., "Mechanics of Bellows: A Critical Review," International Journal of Mechanical Sciences, 1985 (in press).

2. Verma, P. D. S. and Rana, O. H., "Rotation of a Circular Cylindrical Tube Reinforced by Fibres Lying Along Helices," Mechanics of Materials, Vol. 2, Pp. 353-359, 1983.

3. Reissner, E., "On Uniform Stress and Strain in Axially Homogeneous Cylindrical Shells," International Journal of Solids and Structures, Vol. 6, pp. 133-138, 1970 .

4. Reissner, E. and Tsai, W. T., "Pure Bencing, Stretching, and Twisting of Anisotropic Cylindrical Shelis," Journal of Applied Mechanics, Vol. 41, pp. $168-174,1974$.

5. Lekhnitskii, S. G., Theory of Elasticity of an Anisotropic Elastic Body, Translated from Russian by P. Fern, halden-Day, Inc., San Francisco, 1963.

6. Timoshenko, S. P. and Woinowsky-Krieger, S., Theory of Platss ard Shells, 2nd Edition, McGraw-Hill, New York, 1959.

7. Sokolnikoff, I. S., Mathematical Theory of Elasticity, and Editicn, McGraw-Hill, New York, 1956.

8. Dahl, N. C., "Toroidal-Shell Expansion Joints," Journal of Applied Mechanics, Vol. 20, pp. 497-503, 1953. 
APPENDIX A

COMPUTATION OF NEUTRAL AXIS

The iteration steps to compute the position of the neutral axis of the composite section of an element, given an end moment $M_{a}$, were briefly outlined in Chapter 2. The iteration steps including various formulae derivations, are now discussed in detail.

To set up the force equilibrium equation for the composite section it is needed to integrate over elemental areas across the section. For the cylindrical part as shown in Figure $2 a$, the area of elemental steps are as follows.

$$
\begin{aligned}
& \text { area of strip } A B C D \text { is } 2 \sqrt{A_{r 1}} d y \\
& \text { area of strip EFGH is } \sqrt{A_{r 1}} d y-\sqrt{A_{r 2}} d y \text {, }
\end{aligned}
$$

where

$$
\begin{aligned}
& A_{r 1}=R_{1}^{2}-\left(R_{1}-y+H_{1}\right)^{2} \\
& A_{r 2}=R_{2}^{2}-\left(R_{1}-y+H_{1}\right)^{2}
\end{aligned}
$$

The iteration steps are

a. Choose an initial value of $H_{1}$ to fix the position of the neutral axis.

b. After the neutral axis position is chosen the radius of curvature $\rho$ of the deflected element is computed. Due to applied end moment, the part of the section above the neutral axis will be in tension, whereas below the 
neutral axis the section will be in compression. Refer to Figures $2 a$ dnd $2 c$. For the linear reinforcement material the stress strain relationship is

$$
\sigma=E \varepsilon
$$

and strain curvature relationship is

$$
\varepsilon=y / \rho
$$

From Equations (A.3) and (A.4) we get

$$
\sigma=E y / \rho
$$

For the nonlinear synthetic material the stress strain relationship is

$$
\sigma=A \varepsilon+B \varepsilon 1 / n
$$

From Equations (A.4) and (A.6) we get

$$
\sigma=A\left(\frac{y}{\rho}\right)+B\left(\frac{y}{\rho}\right)^{1 / n}
$$

Now

$$
F=\text { Force }=\int_{A} \sigma \mathrm{dA}
$$

From Equations (A.5) and (A.8), the compressive force in the lower part of the reinforcement (below the neutral axis) is

$$
F=\int_{H-H_{1}}^{0} E \frac{y}{\rho} b d y=\frac{-E b\left(H-H_{1}\right)^{2}}{2 \rho}
$$

Similarly the tensile force in the upper part of the reinforcement is

$$
F=\int_{0}^{H_{1}} E \frac{y}{p} b d y=\frac{E B H_{1}^{2}}{2 p}
$$

From Equations (A.7) and (A.8), the tensile force in the cylindrical part is

$$
F=\frac{1}{\rho}(Y)+B\left(\frac{1}{\rho}\right)^{1 / n}(I)
$$

where 


$$
\begin{gathered}
r=2 A\left[\int_{H_{1}}^{R_{1}-R_{2}+H_{1}} y \sqrt{A_{r 1}} d y+\int_{R_{1}-R_{2}+H_{1}}^{R_{1}+R_{2}+H_{1}} y\left\{\sqrt{A_{r 1}}-\sqrt{A_{r 2}}\right] d y\right. \\
\left.\quad+\int_{R_{1}+R_{2}+H_{1}}^{2 R_{1}+H_{1}} y \sqrt{A_{r 1}} d y\right]
\end{gathered}
$$

and

$$
\begin{aligned}
I=2\left[\int_{H_{1}}^{R_{1}-R_{2}+H_{1}} y^{1 / n} \sqrt{A_{r 1}} d y+\int_{R_{1}-R_{2}+H_{1}}^{R_{1}+R_{2}+H_{1}} y^{1 / n}\left\{\sqrt{A_{r 1}}-\sqrt{A_{r 2}}\right\} d y\right. \\
\\
\left.\quad+\int_{R_{1}+R_{2}+H_{1}}^{2 R_{1}+H_{1}} \sqrt[1 / n]{A_{r 1}} d y\right]
\end{aligned}
$$

$\gamma$ and $I$ are evaluated using numerical integration techniques.

To have force equilibrium we know that the sum of compressive and tensile forces is equal to zero. Thus from Equations $(A .9),(A .10)$ and $(A .11)$ we get

$$
\begin{aligned}
& -\frac{E b\left(H-H_{1}\right)^{2}}{2 \rho}+\frac{E b H_{1}^{2}}{2 \rho}+\frac{1}{\rho} Y+B\left(\frac{1}{\rho}\right)^{1 / n} I=0 \\
& \frac{E b\left(H^{2}-2 H H_{1}\right)-2 \gamma}{2 \rho}=B\left(\frac{1}{\rho}\right)^{1 / n} I \\
& \frac{E b\left(H^{2}-2 H H_{1}\right)-2 \gamma}{2 B I}=\left(\frac{1}{\rho}\right)^{\frac{1}{n}-1} \\
& \frac{1}{\rho}=\left[\frac{E b\left(H^{2}-2 H H_{1}\right)-2 \gamma}{2 B I}\right]^{\frac{n}{1-n}} \\
& \rho=\left[\frac{2 B I}{E b\left(H^{2}-2 H H_{1}\right)-2 \gamma}\right]^{\frac{n}{1-n}}
\end{aligned}
$$


c. For the computed radius of curvature $\rho$ and chosen position of neutral axis the moment of resistance of the section $M_{c}$ is evaluated.

$$
\text { Moment of resistance }=\int_{A} \text { oy } d A
$$

Using Equations (A.5) and (A.15) the moment resisted by the reinforcement is

$$
\begin{gathered}
M_{r}=\int_{0}^{H_{1}} y^{2} \frac{E}{\rho} b d y+\int_{H_{1}-H}^{0} y^{2} \frac{E}{\rho} b d y \\
M_{r}=\frac{1}{\rho}(\alpha)
\end{gathered}
$$

where

$$
\alpha=\frac{E b}{3}\left[H_{1}^{3}+\left(H-H_{1}\right)^{3}\right]
$$

Using Equations (A.7) and (A.15) the moment resisted by the nonlinear cylindrical part is

$$
M_{n}=\left(\frac{1}{\rho}\right) B+\left(\frac{1}{\rho}\right)^{1 / n_{n}}
$$

where

$$
\begin{aligned}
B & =\underset{H_{1}}{2 A\left[\int_{1}^{1}-R_{2}+H_{1}\right.} y^{2} \sqrt{A_{r 1}} d y+\int_{R_{1}-R_{2}+H_{1}}^{R_{1}+R_{2}+H_{1}} y^{2}\left[\sqrt{A_{r 1}}-\sqrt{A_{r 2}}\right\} d y \\
& \left.+\int_{R_{1}+R_{2}+H_{1}}^{2 R_{1}+H_{1}} y^{2} \sqrt{A_{r 1}} d y\right]
\end{aligned}
$$

and

$$
\begin{aligned}
n & =2 B\left[\int_{H_{1}}^{R_{1}-R_{2}+H_{1}} y^{\frac{n+1}{n}} \sqrt{A_{r 1}} d y+\int_{R_{1}-R_{2}+H_{1}}^{R_{1}+R_{2}+H_{1}} y^{\frac{n+1}{n}}\left(\sqrt{A_{r 1}}-\sqrt{A_{r 2}} d y\right.\right. \\
& \left.+\int_{R_{1}+R_{2}+H_{1}}^{2 R_{1}+H_{1}} y^{\frac{n+1}{n}} \sqrt{A_{r 1}} d y\right]
\end{aligned}
$$

The total moment resisted by the section 


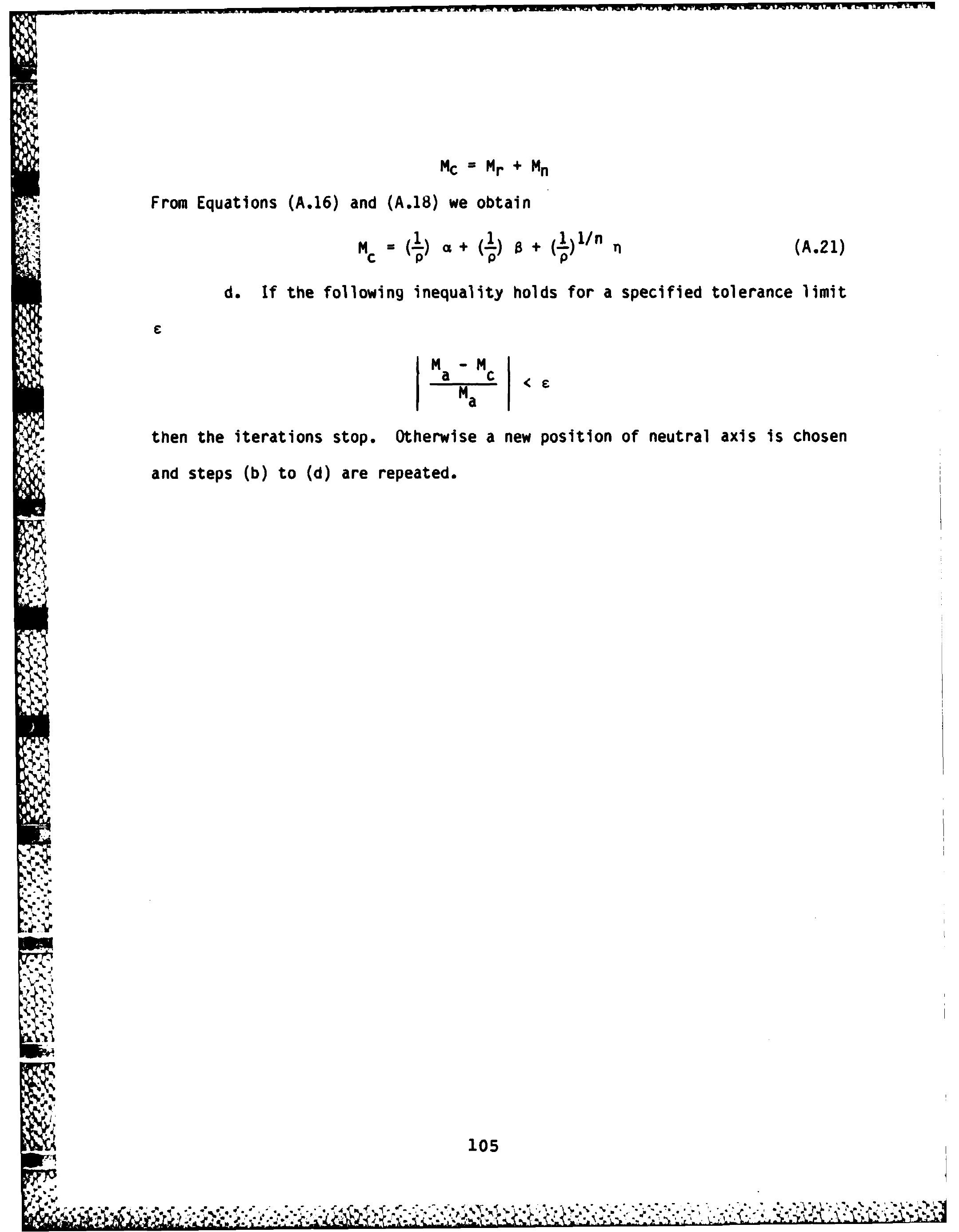


APPENDIX B: PROGRAM LISTINGS

PROGRAM TYPEL

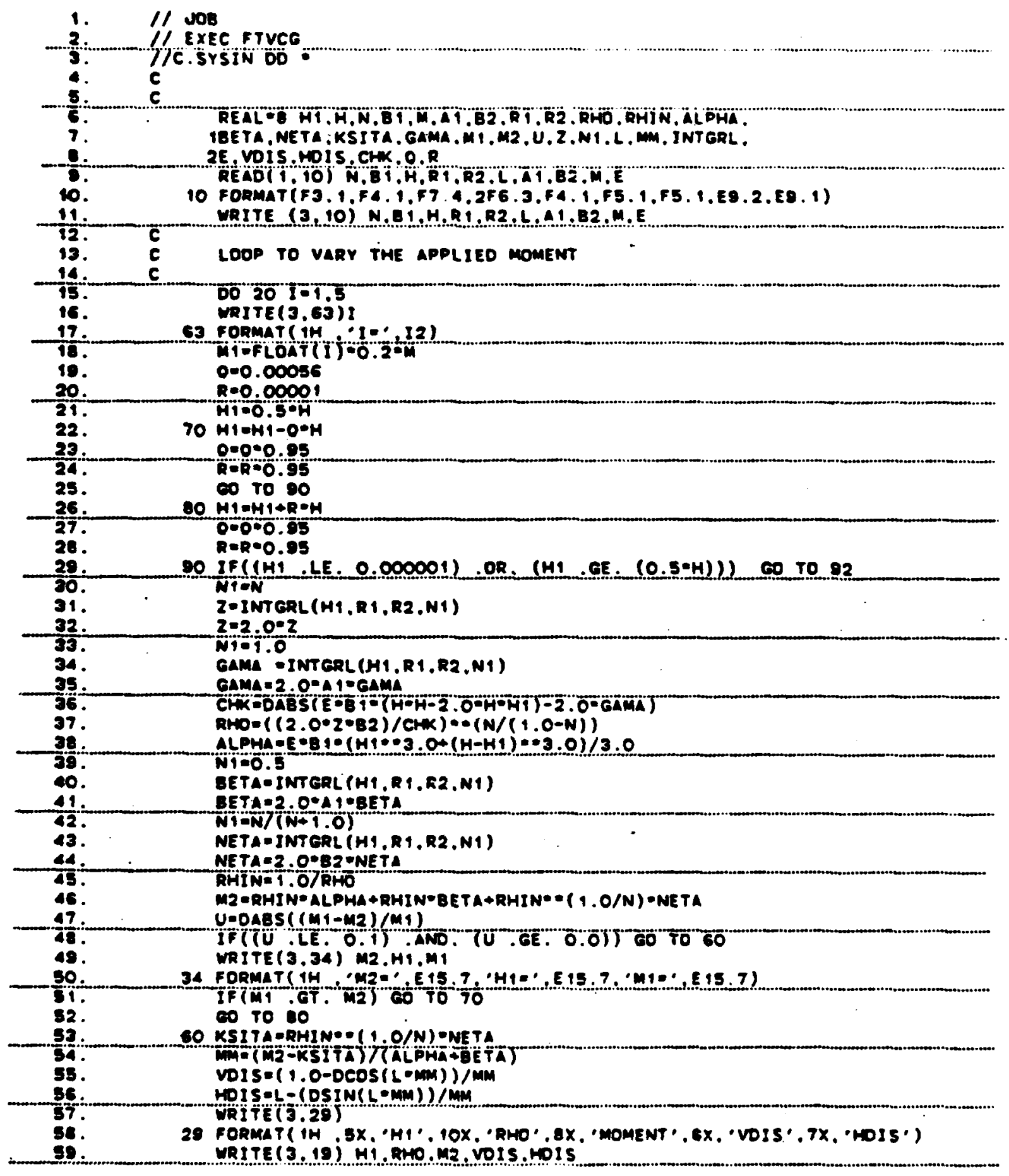




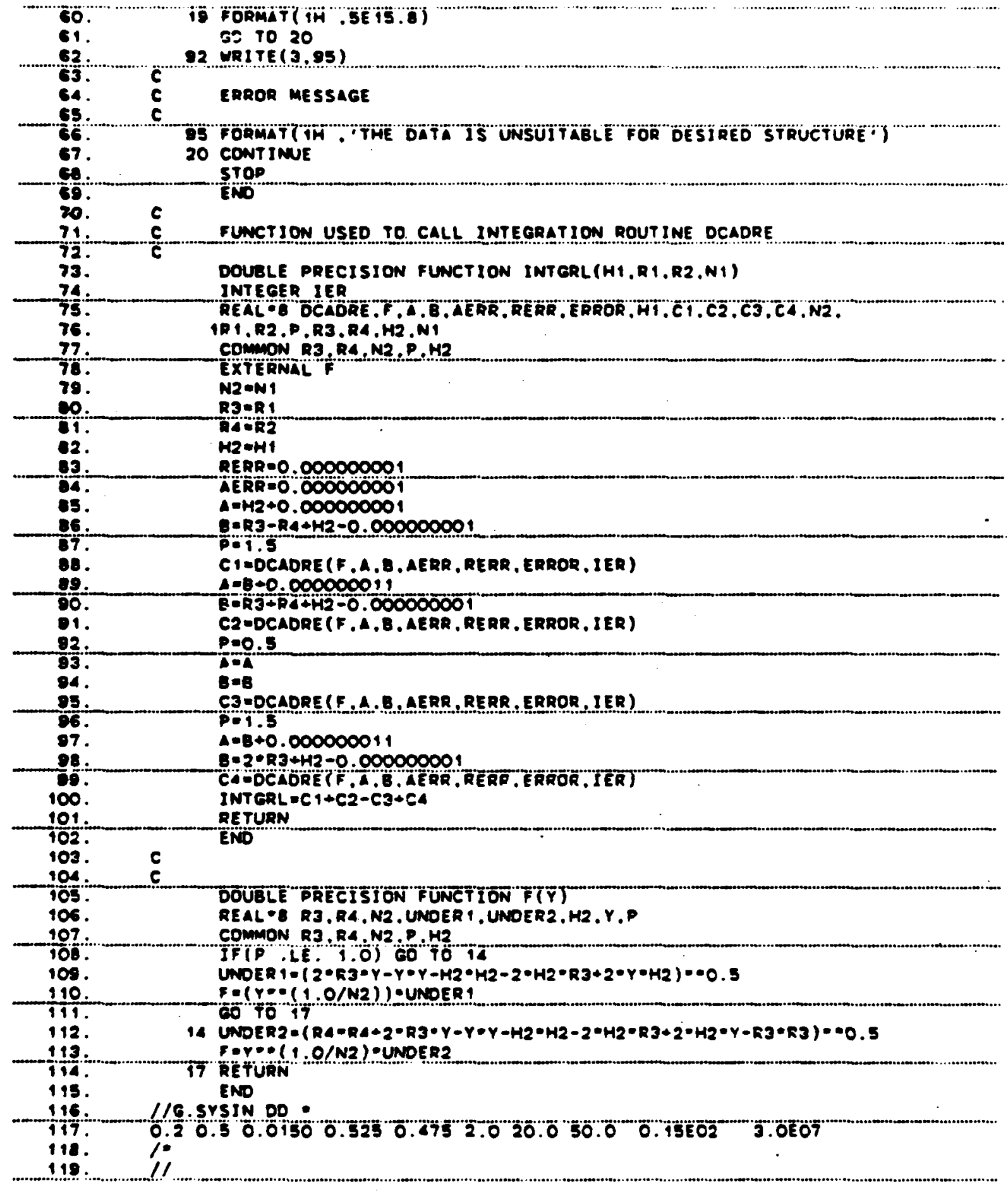




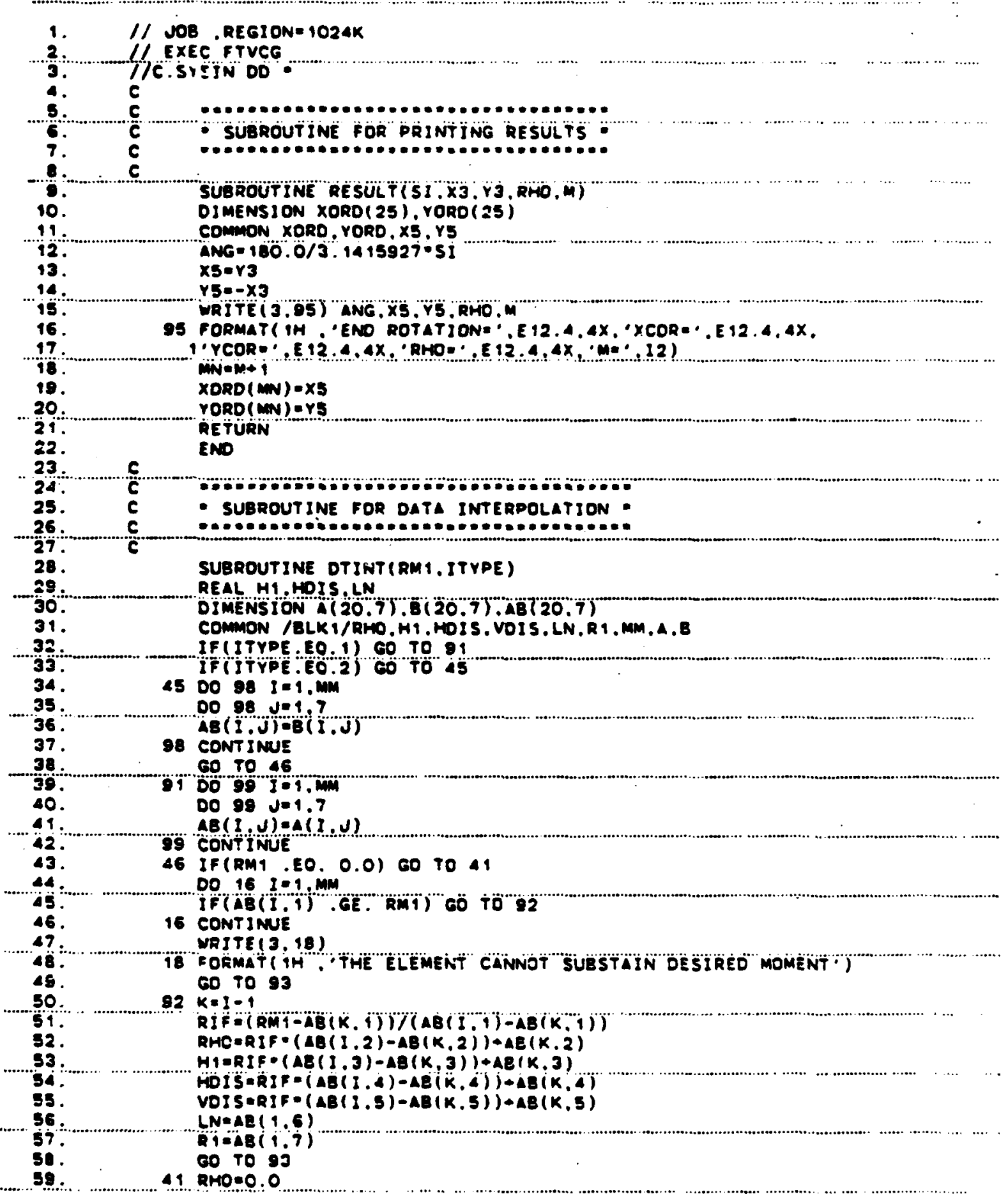




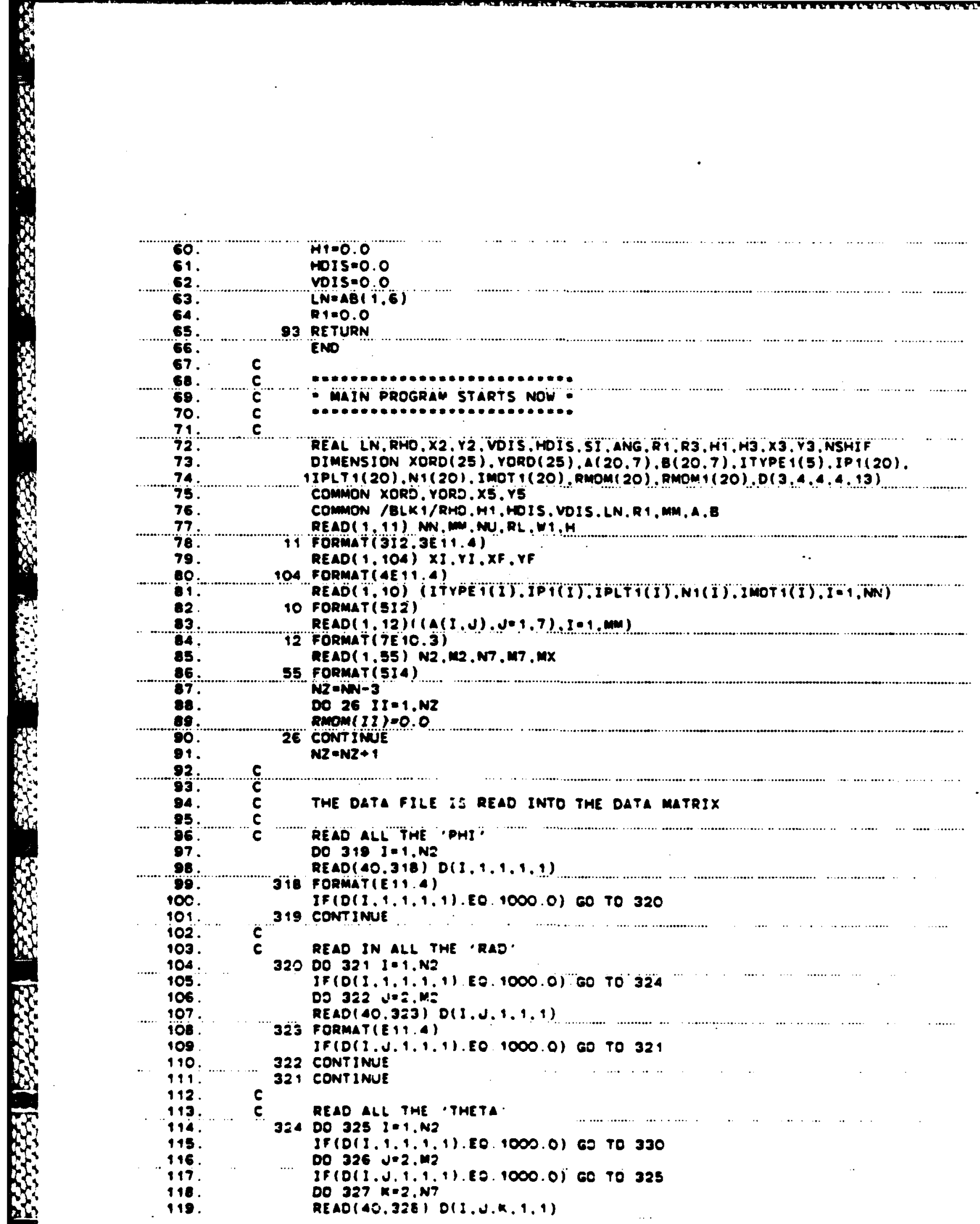




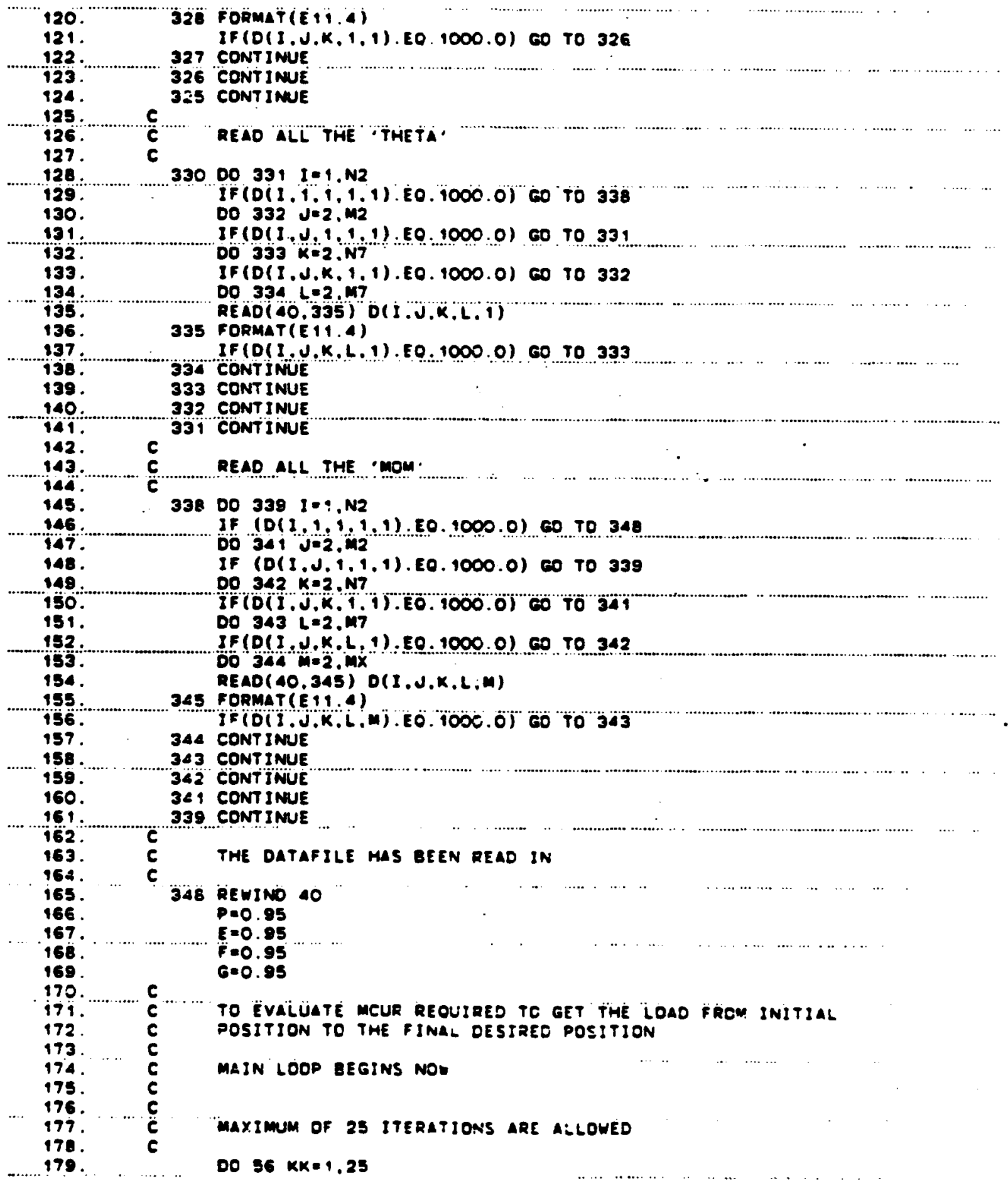




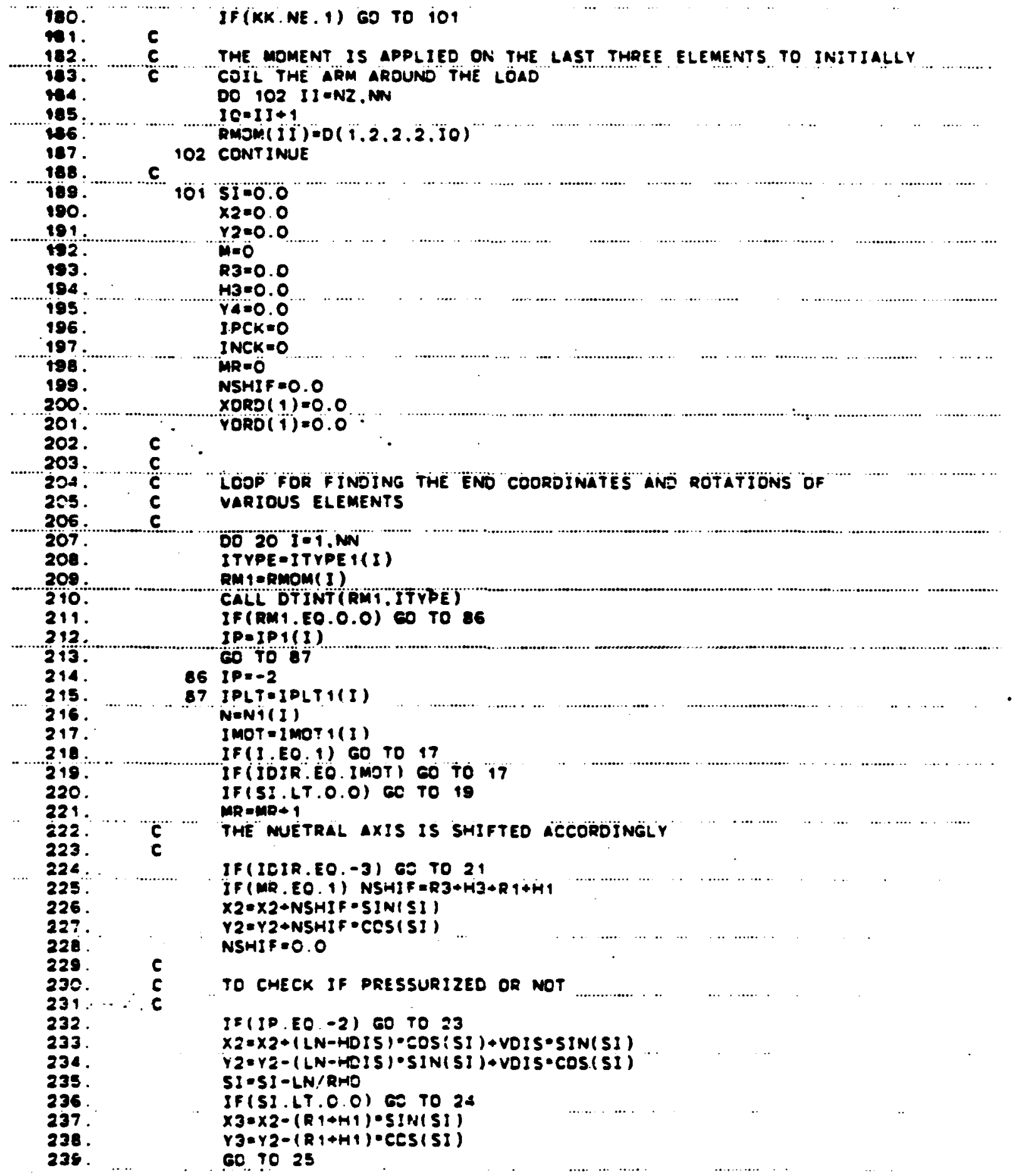




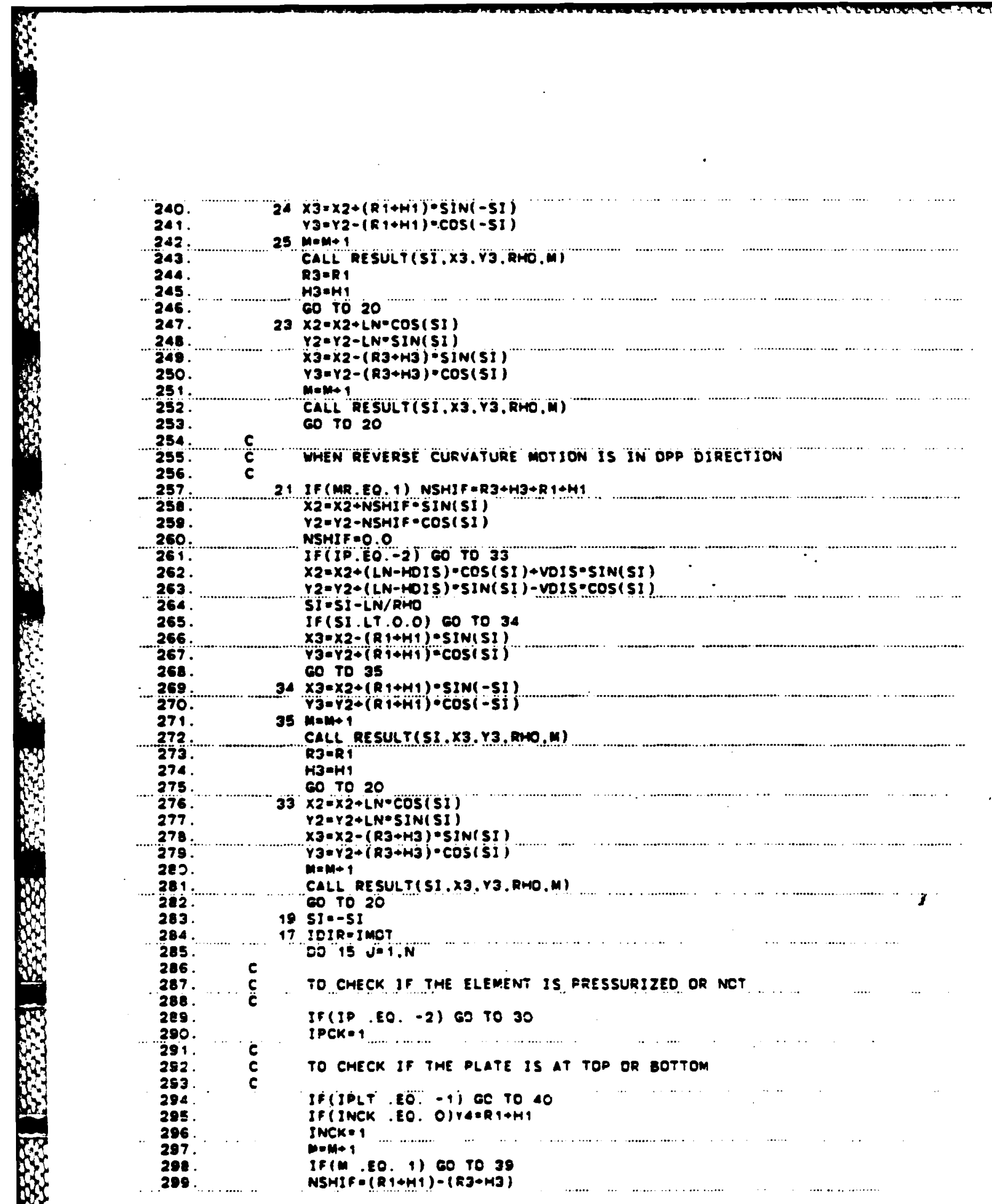




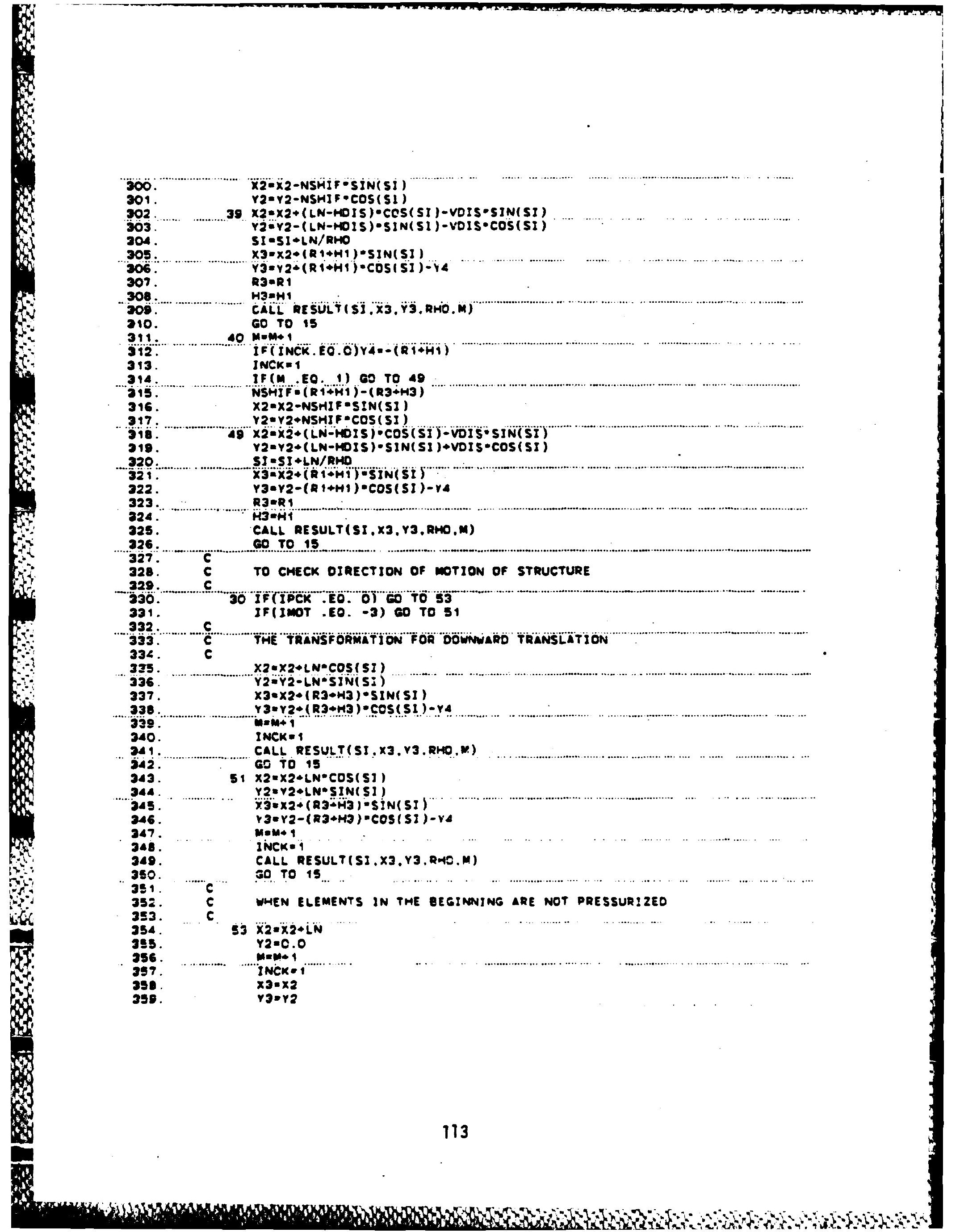




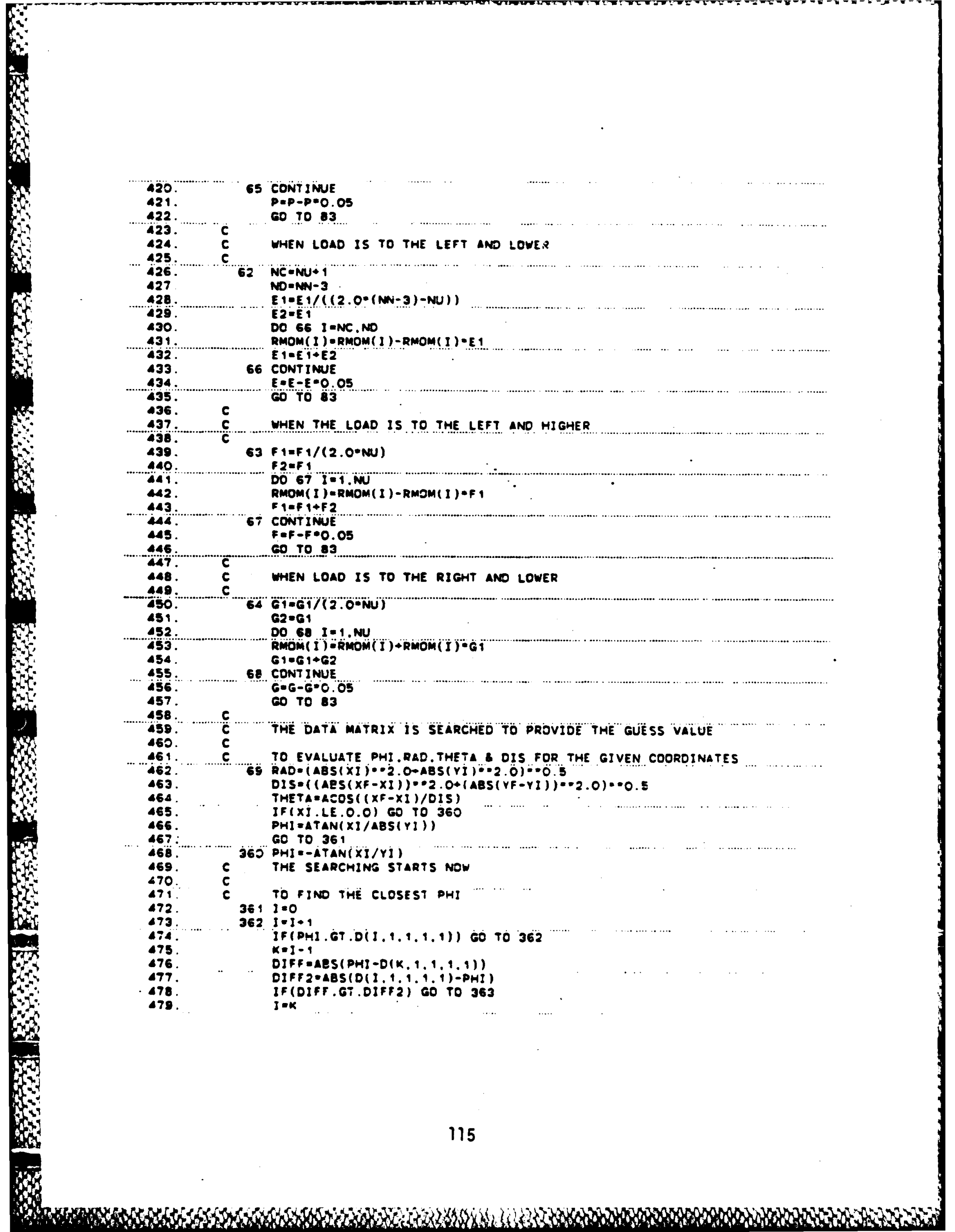

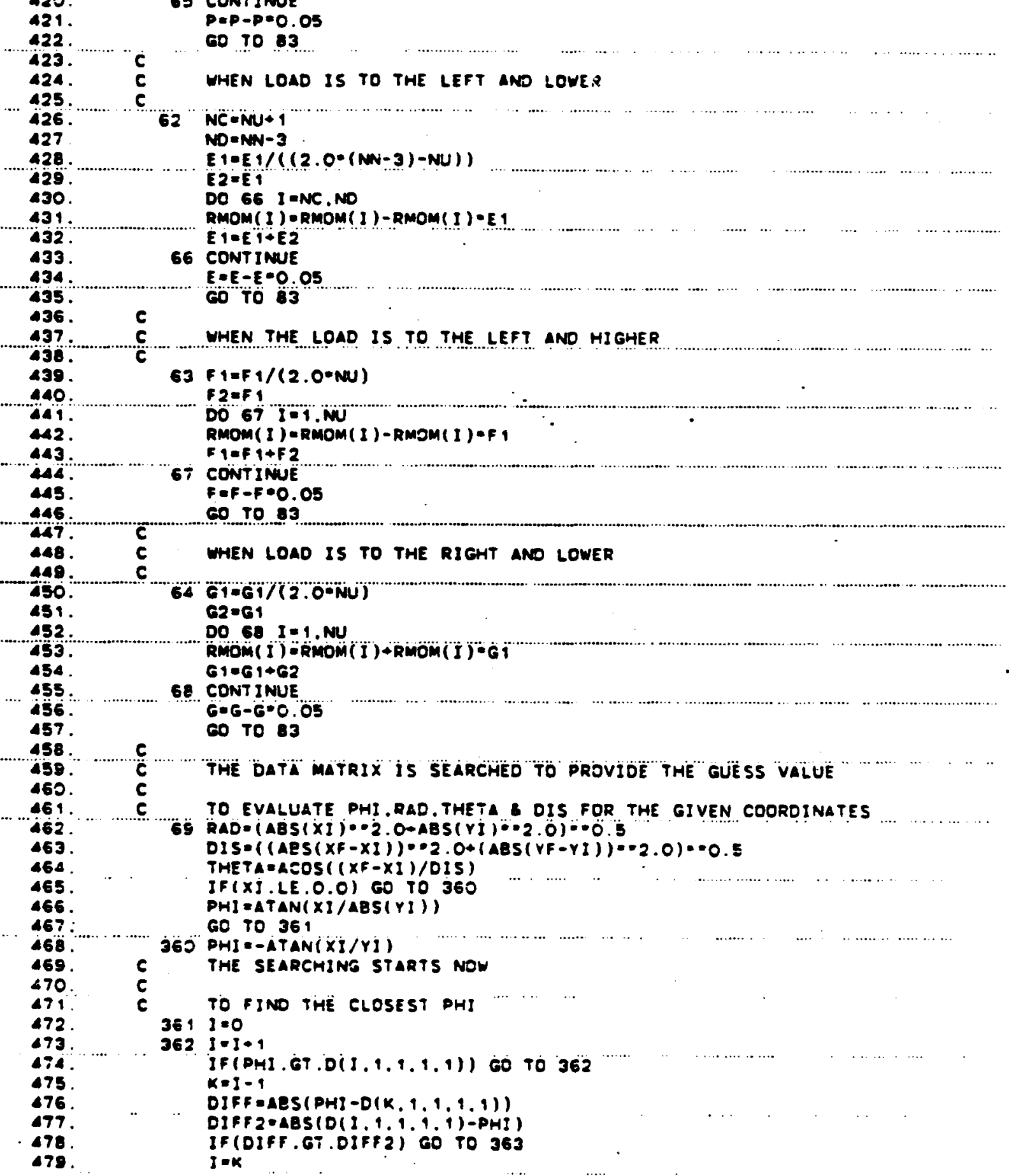




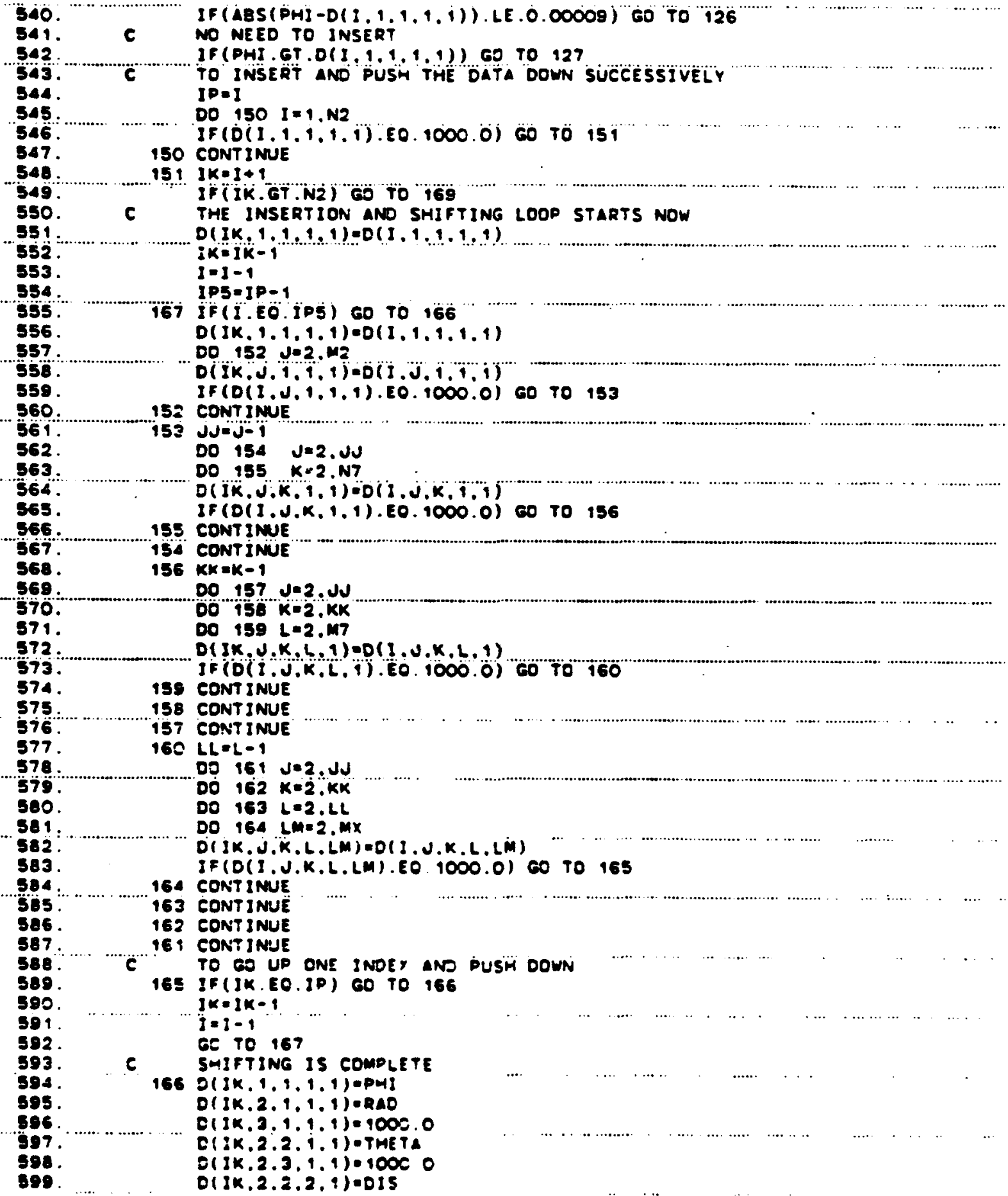




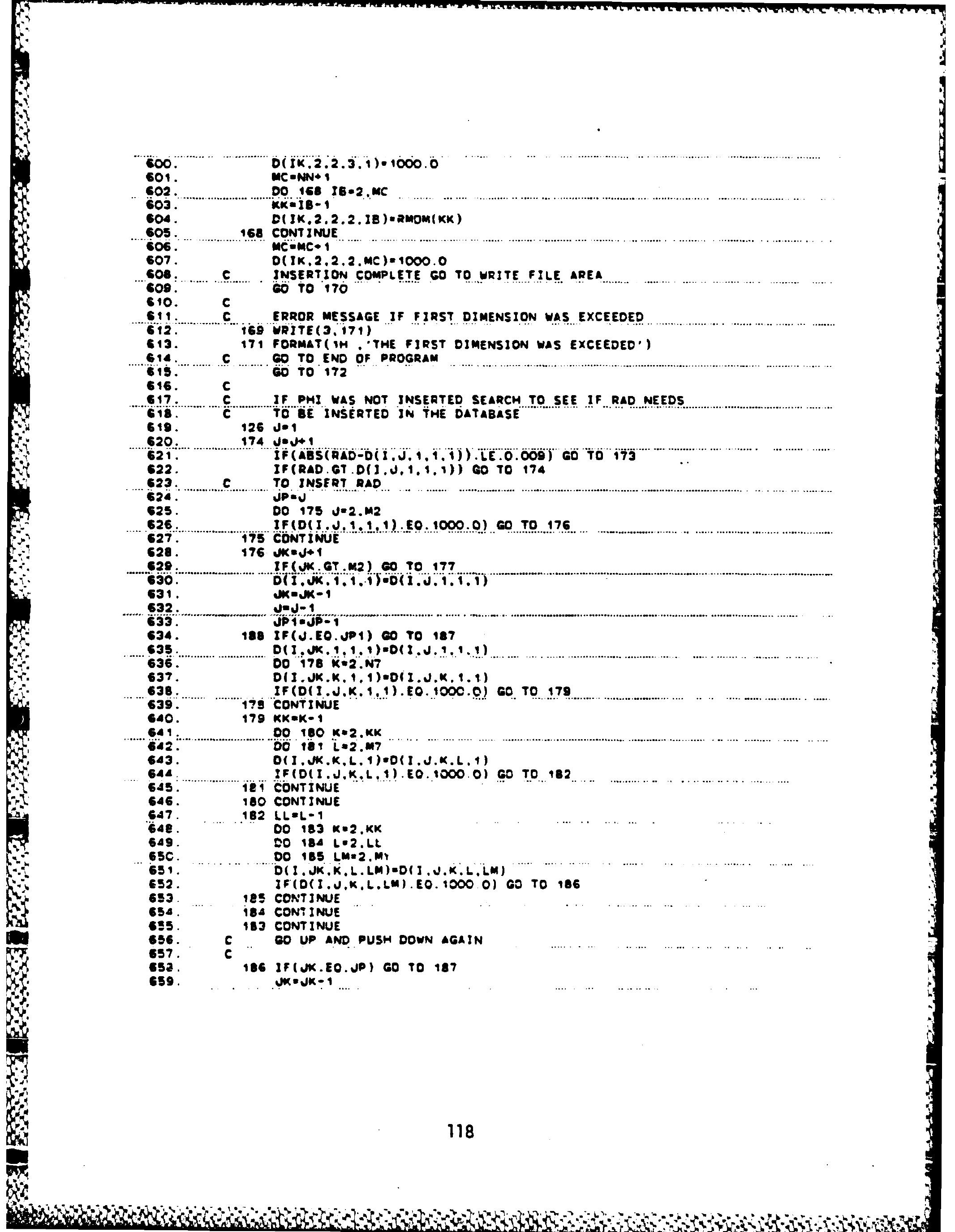




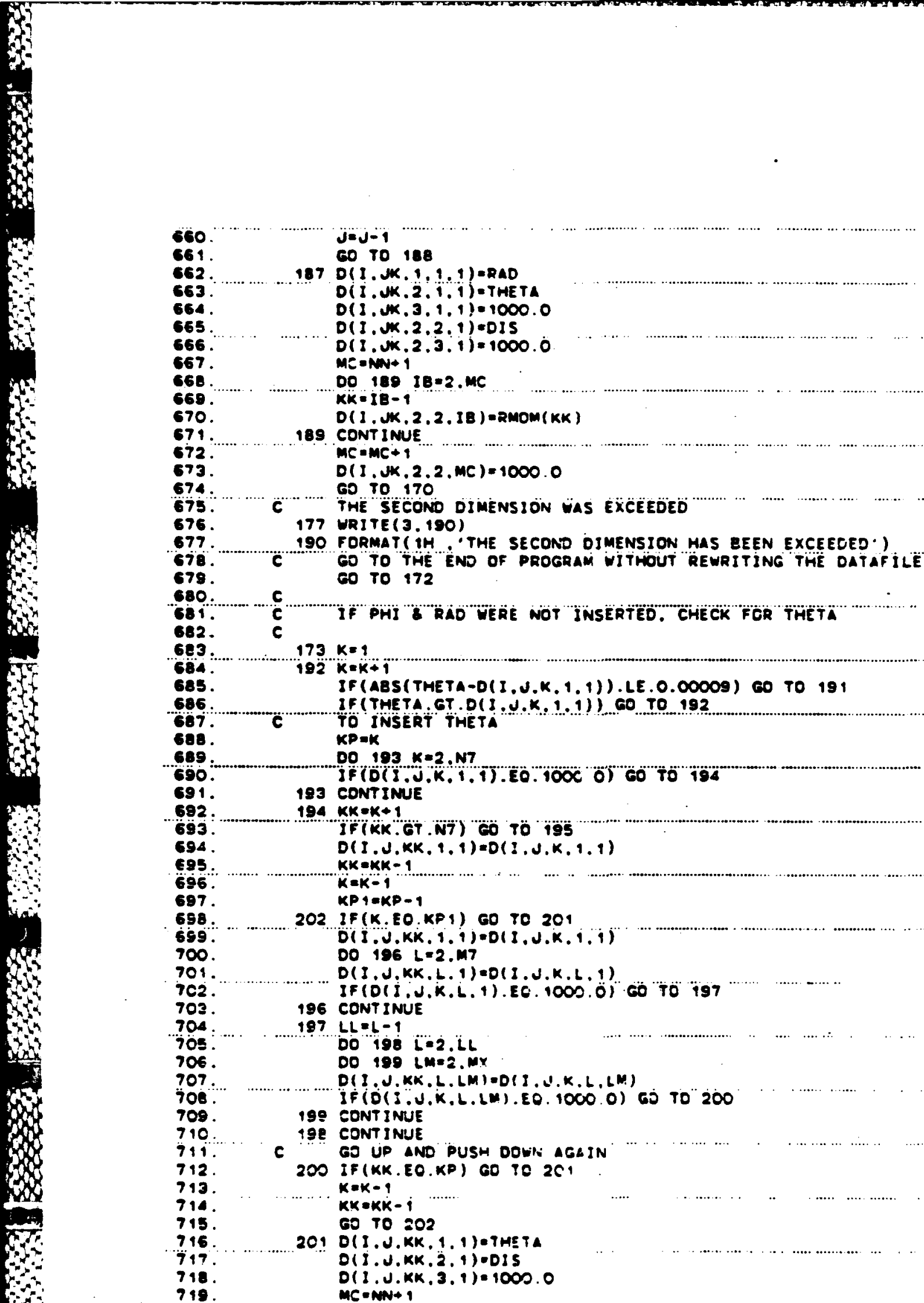

674.

675.

676.

677.

678.

679.

s80.

Gei.

682.

683.

684.

685.

686.

687.

688.

689.

600 .

691.

692.

693.

694.

egs

696.

697.

658.

659.

700.

701.

$7 \mathrm{Cs}$

703.

704.

705

706.

707.

708.

709.

710.

741.

712

713.

714.

715.

716

747

718.

710

$j=j-1$

6o To 188

$187 \mathrm{D}(1 . J K .1,1.1)=$ RAD

D(i.UK.2.1.,i)=THETA

D(1.UK.3.1.1)=1000.0

$0(1 . J K .2,2.1)=015$

$O(1 . J K .2 .3 .1)=1000.0$

$M E=N N+1$

Do $18018=2 . M C$

KK $=18-1$

$D(1, U K, 2,2, I B)=R M O M(K K)$

189 CONT INUE

$M C=M C+1$

$D(1, J K, 2,2, M C)=1000.0$

60 To 170

C.... THE SECOND DIMENS IÖN V̈AS EXCEEEÖED

177 WRITE (3.190)

190 FORMAT( IH. - THE SECOND DIMENSION HAS EEEN EXCEECED')

C GO TO THE END OF PROGRAM VITHOUT REWRITING THE DATAFILE

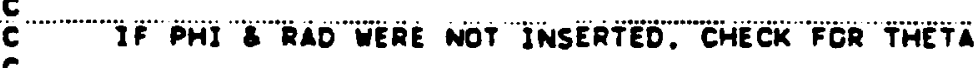

$173 k=1$

$192 k=k+1$

IF(ABS(THETA-D(I,J.K, I, 1)).LE.0.00009) GO TO 191

IF(THETA.GT.O(I.U.K.1.1)) CO TO 192

C TO INSERT THETA

KP=K

Do $193 K=2, N 7$

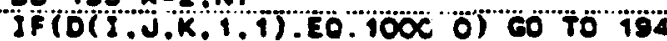

193 CONTINUE

$194 K K=K+1$

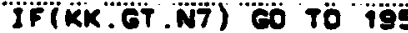

$D(I . J, K K, 1,1)=0(1, J, K, 1,1)$

KK=KK- 1

$k=k=i$

KP I $K$ KP-1

202 IF(K.EO.KP1) CO TO 201

$D(j, j, K K, 1, i)=D(1, J, K, i, i)$

DO $196 \quad L=2 . M 7$

$D($ I.J.KK.L.I) $0($ I.J.K.L.1)

IF(O(j.J.K.L.1) EC.1000.0) 607099

196 CONT INUE

$197 L L=L-1$

Do $198 \quad I=2 . L L$

DO 190 L $M=2 . M X$

$D(1, J, K K, L, L M)=0(1,1, K, L, L M)$

IF(O) :.J.K.L.LN).EO. 1000.0) CS TO" 200

199 CONTINUE

192 CONT INUE

c

GO UP AND PUSH DOVII $\triangle G A I N$

200 IF (KK.EO.KP) 00 TO $2 \mathrm{C}$

$k=k-?$

kK=kK-i

6o 70202

201 O(I.J.KK, 1.1)=THETA

O(1.J.KK.2.,1)=01s

D (1.J.KK, 3.1)=1000.0

$M C=N N+1$ 


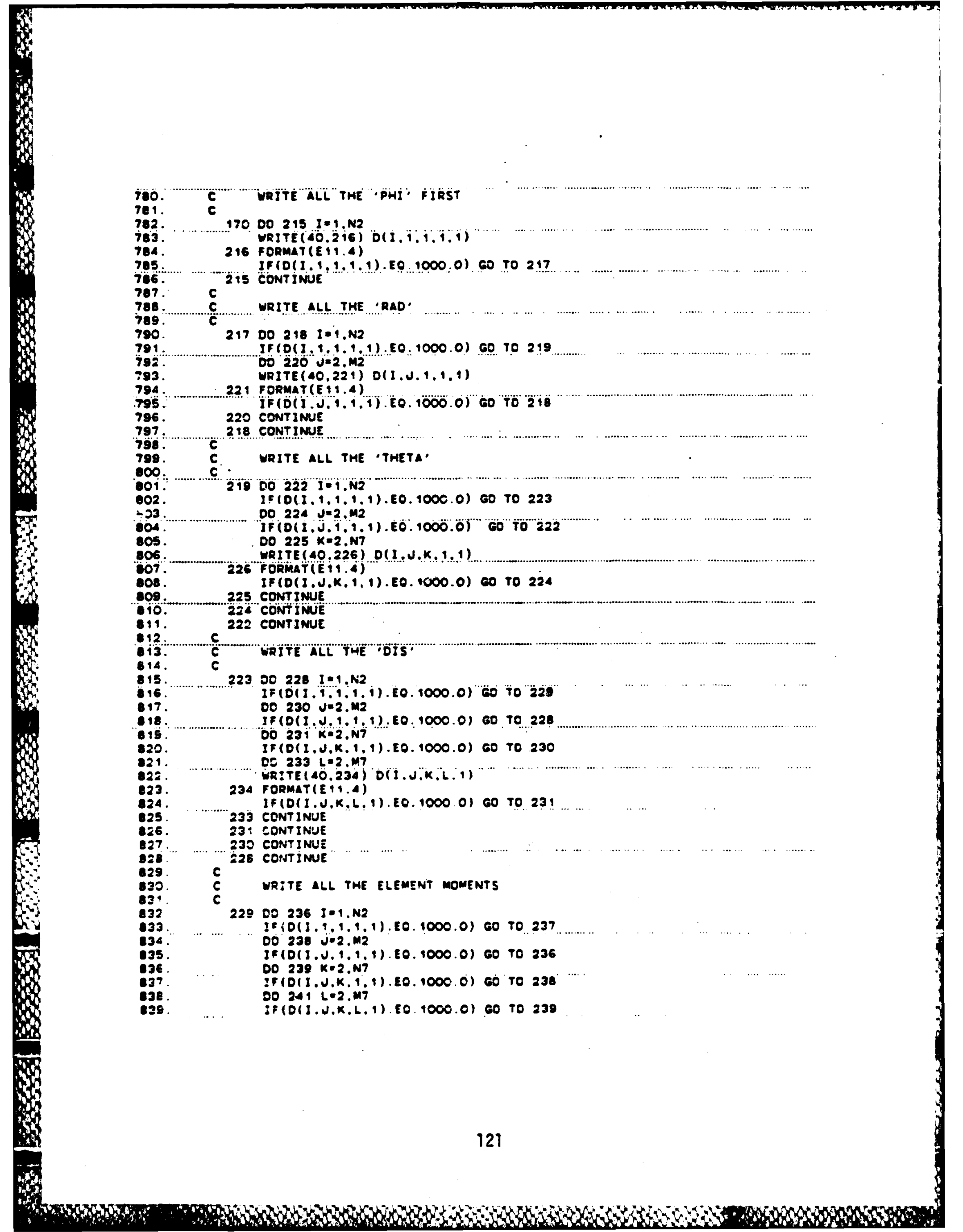




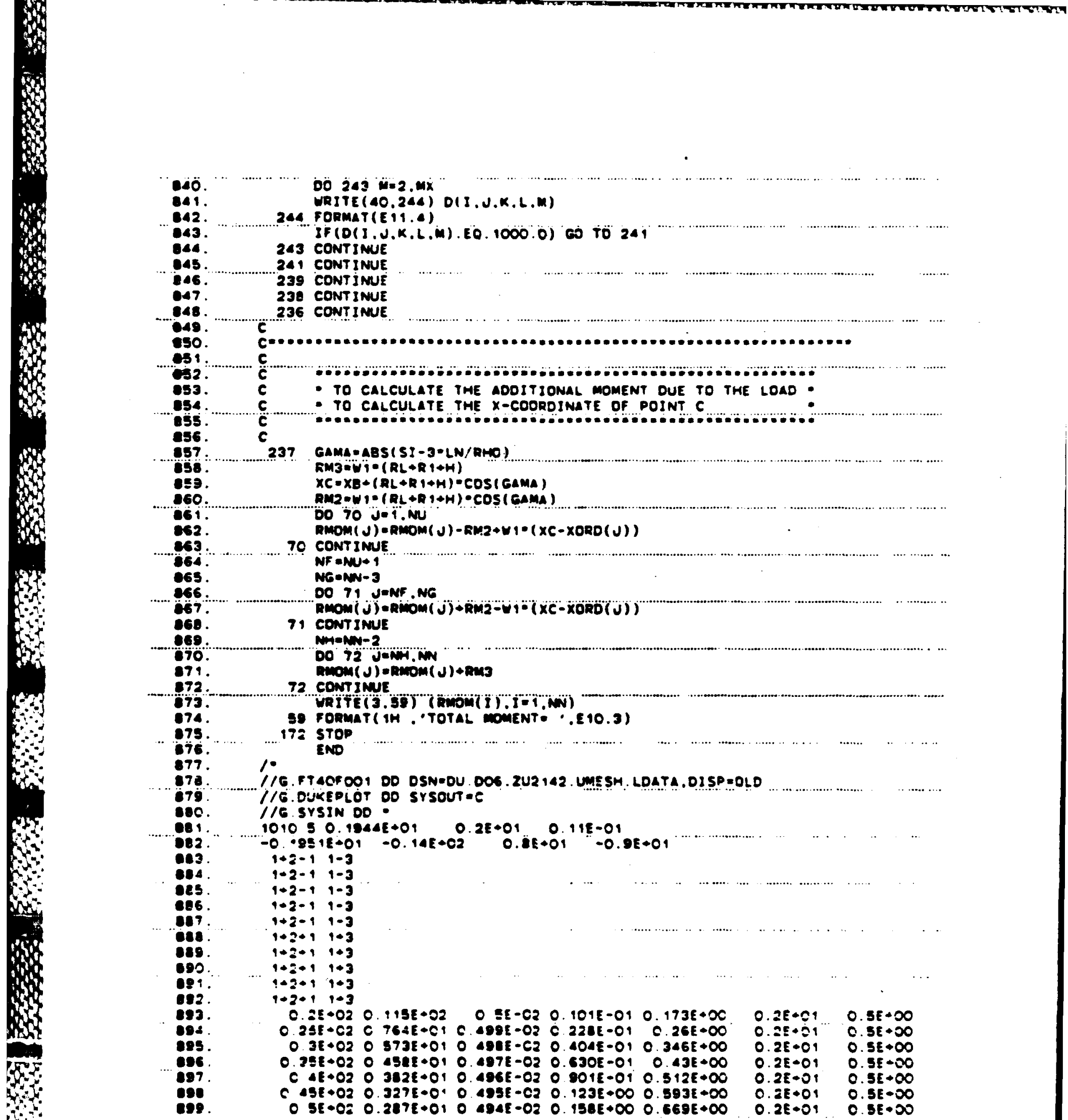


